\title{
WEIGHTED K-STABILITY OF POLARIZED VARIETIES AND EXTREMALITY OF SASAKI MANIFOLDS
}

\author{
VESTISLAV APOSTOLOV, DAVID M. J. CALDERBANK, AND EVELINE LEGENDRE
}

\begin{abstract}
We use the correspondence between extremal Sasaki structures and weighted extremal Kähler metrics defined on a regular quotient of a Sasaki manifold, established by the first two authors in 1], and Lahdili's theory of weighted K-stability [40] in order to define a suitable notion of (relative) weighted K-stability for compact Sasaki manifolds of regular type. We show that the (relative) weighted K-stability with respect to a maximal torus is a necessary condition for the existence of a (possibly irregular) extremal Sasaki metric. We also compare weighted K-stability to the K-stability of the corresponding polarized affine cone (introduced by Collins-Székelyhidi in [18]), and prove that they agree on the class of test configurations we consider. As a byproduct, we strengthen the obstruction to the existence of a scalar-flat Kähler cone metric found in [18] from the K-semistability to the K-stability on these test configurations. We use our approach to give a characterization of the existence of a compatible extremal Sasaki structure on a principal $\mathbb{S}^{1}$-bundle over an admissible ruled manifold in the sense of [4, expressed in terms of the positivity of a single polynomial of one variable over a given interval.
\end{abstract}

\section{INTRODUCTION}

The famous Calabi problem [15], which seeks the existence of canonical Kähler metrics in a given Kähler class $\zeta \in H^{2}(M, \mathbb{R})$ on a compact complex manifold $(M, J)$, is a central and very active topic of current research in Kähler geometry. As a candidate for a canonical metric Calabi proposed a notion of extremal Kähler metric g, meaning that its scalar curvature $\operatorname{Scal}(g)$ is a Killing potential, i.e., the vector field $\operatorname{Jgrad}_{g} \operatorname{Scal}(g)$ is a Killing vector field for $g$. Examples include constant scalar curvature (CSC) Kähler metrics on $(M, J)$, and hence also Kähler-Einstein metrics.

There is an odd-dimensional analogue of the Calabi problem-and a corresponding notion of extremal Sasaki structures (called canonical in [11]) - associated to compact, strictly pseudoconvex CR manifolds $(N, \mathcal{D}, J)$ of Sasaki type. This may be motivated using the construction of regular Sasaki structures on the total space $N$ of the principal $\mathbb{S}^{1}$-bundle over a Hodge manifold $(M, J, \zeta)$ determined by the given integral Kähler class $\zeta / 2 \pi \in H^{2}(M, \mathbb{Z})$ : any Kähler metric $\omega \in \zeta$ on $M$ induces a connection 1-form $\eta$ on $N$ satisfying $d \eta=\pi^{*} \omega$, and hence a $\mathrm{CR}$ structure $(\mathcal{D}, J)$ with $\mathcal{D}=\operatorname{ker} \eta \subseteq T N$ and $J$ the pullback of the complex structure on $M$ to $\mathcal{D} \cong \pi^{*} T M$. In this case, the vector field $K$ induced by the fibrewise $\mathbb{S}^{1}$-action on $N$ is called regular Sasaki-Reeb vector field. The Calabi problem on $(M, J, \zeta)$ translates to the search for CR structures $(\mathcal{D}, J)$ on $N$, compatible with the induced transversal holomorphic structure $J^{K}$ on the vector bundle $T N /\langle K\rangle \rightarrow N$, such that $(\mathcal{D}, J, K)$ is a Sasaki structure corresponding to an extremal, CSC or Kähler-Einstein metric $\omega \in \zeta$ on $M$. This leads respectively to notions of extremal, CSC and Einstein Sasaki structures on $N$.

The above construction can be generalized by relaxing the assumption that the $\mathbb{S}^{1}$-action on $N$ generated by the vector field $K$ is free. If it is instead required only to be locally free, then $N$ can be realized as a principal $\mathbb{S}^{1}$-orbibundle over a kählerian orbifold $(M, J, \zeta)$, and $K$ is then referred to as a quasiregular Sasaki-Reeb vector field on $N$. One can also

Date: December 17, 2020. 
weaken the assumption that $K$ generates an $\mathbb{S}^{1}$-action, and require only that the closure of the subgroup generated by the vector field $K$ is a compact torus $\mathbb{T}$ in the diffeomorphism group of $N$ preserving the given transversal holomorphic structure $J^{K}$; if $\operatorname{dim} \mathbb{T}>1, K$ is called an irregular Sasaki-Reeb vector field.

Irrespective of regularity, the correspondence between Kähler geometry and Sasaki geometry holds locally, allowing one to extend the notions of extremal, CSC and Einstein Sasaki structures: indeed, any point of a Sasaki manifold $(N, \mathcal{D}, J, K)$ has a neighbourhood in which the leaf space $M$ of the flow of $K$ is a manifold and has an induced Kähler structure $(g, J, \omega)$. We refer to $(M, g, J, \omega)$ as a Sasaki-Reeb quotient of $(N, \mathcal{D}, J, K)$, and say that $(N, \mathcal{D}, J, K)$ is extremal, CSC or Sasaki-Einstein if any local Sasaki-Reeb quotient is extremal, CSC, or Kähler-Einstein manifold, respectively.

The study of irregular extremal Sasaki metrics is more delicate than the regular case (which reduces to the Calabi problem in the Kähler context). One approach for dealing with the issue of irregularity stems from the seminal work of Martelli-Sparks-Yau [47, who showed that the existence of a Sasaki-Einstein metric compatible with a given transversal holomorphic structure $\left(K, J^{K}\right)$ on $N$ can be equivalently studied using strictly plurisubharmonic smooth positive functions on the (noncompact) smooth manifold $V=\mathbb{R}^{+} \times N$ equipped with a conical complex structure $J$ inducing the transversal holomorphic structure $\left(K, J^{K}\right)$; such functions define Kähler metrics on $(V, J)$ which are Ricci-flat if and only if the induced Sasaki structure on $N$ is Einstein. Furthermore, they observed that $(V, J, K)$ can be given the structure of an affine cone embedded in $\mathbb{C}^{k}$ for some $k \gg 0$, with $K$ being in the Lie algebra of a maximal compact algebraic torus $\mathbb{T} \leqslant \mathrm{GL}(k, \mathbb{C})$ preserving $(V, J)$. The framework of [47] can be adapted [36, 18, 14] to recast, more generally, the search for compatible extremal Sasaki structures on $\left(N, K, J^{K}\right)$.

Using the algebraic nature of $(V, J, K)$, Collins-Székelyhidi [18] introduced a suitable notion of $K$-stability of $(V, J, K, \mathbb{T})$, and showed that the $K$-semistability of $(V, J, K, \mathbb{T})$ is a necessary condition for the existence of a compatible CSC Sasaki structure on $\left(N, K, J^{K}\right)$. A corresponding notion of relative $\mathrm{K}$-stability for $(V, J, K, \mathbb{T})$, obstructing the existence of extremal Sasaki structures on $\left(N, K, J^{K}\right)$, has been introduced in [14], where the relative $\mathrm{K}$-semistability of $(V, J, K, \mathbb{T})$ with respect to $\mathbb{T}$-equivariant test configurations of affine cones in $\mathbb{C}^{k}$ is shown to be a necessary condition for the existence of a compatible extremal Sasaki structure on $\left(N, K, J^{K}\right)$. These works suggest a general Yau-TianDonaldson correspondence - analogous to the projective Kähler case [25, 55, 61] - for the existence of compatible extremal Sasaki structures on $\left(N, K, J^{K}\right)$ expressed in terms of a suitable notion of K-stability of the affine complex cone $(V, J, K)$. Such a correspondence has only been established in the Sasaki-Einstein case in [19], where Collins and Székelyhidi refine their original notion of K-stability by considering compatible special test configurations of $(V, J, K, \mathbb{T})$, show that the existence of a compatible Sasaki-Einstein metric implies that $(V, J, K, \mathbb{T})$ is $\mathrm{K}$-stable (not merely $\mathrm{K}$-semistable) for such test configurations and, conversely, prove that the $\mathrm{K}$-stability of $(V, J, K, \mathbb{T})$ with respect to special test configurations yields the existence of a Sasaki-Einstein metric on $\left(N, K, J^{K}\right)$. None of these refinements seem to be available in the general CSC and extremal cases.

Thus motivated, in this paper we propose and develop a new approach to the existence problem of extremal Sasaki structures and the corresponding notion of (relative) K-stability. It uses the key feature (see [17, 29, 42, 46]) that one can construct (even irregular) CSC Sasaki manifolds from Kähler manifolds which are not necessarily CSC. This follows from the observation that the space of Sasaki-Reeb vector fields $K$ (i.e., the vector fields giving rise to transversal holomorphic structures of Sasaki type) is open in the Lie algebra $\mathfrak{t}$ of a compact torus $\mathbb{T}$ inside the group of $\mathrm{CR}$ transformation of $(N, \mathcal{D}, J)$. In particular, one can consider two different Sasaki-Reeb vector fields $X, K \in \mathfrak{t}$, such that 
$X$ is quasiregular even if $K$ is not. We will focus in this paper on the case that $X$ is a regular Sasaki-Reeb vector field, although some of our considerations can be extended to the case of quasiregular $X$, with the obvious technical difficulties coming from dealing with orbifolds rather than smooth manifolds.

A correspondence between Sasaki structures. Suppose therefore that $(N, \mathcal{D}, J, X)$ is a regular compact Sasaki manifold over a smooth polarized Kähler manifold $(M, J, L)$, with induced Sasaki-Reeb quotient Kähler structure $(g, \omega)$ on $M, \omega \in \zeta=2 \pi c_{1}(L)$, and suppose also that $K$ is another Sasaki-Reeb vector field on $(N, \mathcal{D}, J)$ commuting with $X$. Then $K$ projects to a Killing vector field on $(M, J, g, \omega)$, denoted by $\check{K}$, which (by the Sasaki-Reeb condition) has a positive Killing potential $f$ with respect to $g$. Furthermore, as observed in [1], $(\mathcal{D}, J, K)$ is an extremal Sasaki structure if and only if $(g, \omega)$ satisfies the condition that $(m+2, f)$-weighted scalar curvature

$$
\operatorname{Scal}_{f, m+2}(g):=f^{2} \operatorname{Scal}(g)-2(m+1) f \Delta_{g} f-(m+2)(m+1)|d f|_{g}^{2}
$$

of $g$ is a Killing potential; here $m$ is the complex dimension of $M$, and $\operatorname{Scal}(g)$ and $\Delta_{g}$ are the scalar curvature and riemannian Laplacian of $g$. We refer herein to such an $(m+2, f)$ extremal Kähler metric as a $K$-extremal Kähler metric: see $\$ 3.2-\$ 3.3$ for more details.

Following the approach in [5], one can extend the Calabi problem to this (more general) weighted setting, and relate it to the extremal Sasaki problem as follows.

Proposition 1 (see Proposition 3.7). Let (N, D, J,X) be a compact quasiregular Sasaki manifold, and $(M, J, L)$ the corresponding smooth polarized Kähler orbifold. Suppose that $K$ is a Sasaki-Reeb vector field on $(N, \mathcal{D}, J)$ which commutes with $X$. Then $N$ admits an extremal Sasaki structure compatible with the transversal holomorphic structure $\left(K, J^{K}\right)$ determined by $(K, \mathcal{D}, J)$ if and only if $(M, J)$ admits a $K$-extremal Kähler metric in the Kähler class $2 \pi c_{1}^{\text {orb }}(L)$.

A weighted K-stability theorem. The class of $K$-extremal Kähler metrics is a special case of a more general notion of weighted extremal Kähler metrics studied recently by Lahdili [38, 39, 40]. In particular, it follows from these works that one can reduce the search for $K$-extremal metrics on a smooth polarized compact Kähler manifold $(M, J, L)$ by fixing a maximal compact torus $\mathbb{T} \leqslant \operatorname{Aut}(M, L)$, such that $K$ belongs to Lie $(\mathbb{T})$, and considering $\mathbb{T}$-invariant Kähler metrics in $\zeta=2 \pi c_{1}(L)$. Furthermore, for any smooth $\mathbb{T}$ equivariant polarized test configuration $(\mathscr{M}, \mathscr{L})$ of $(M, L)$, Lahdili [40] associates a notion of weighted Donaldson-Futaki invariant $\mathscr{F}_{K}^{\operatorname{ext}}(\mathscr{M}, \mathscr{L})$ with respect to the Sasaki-Reeb vector field $K$, and proves that if $(M, J)$ admits a $K$-extremal Kähler metric in $\zeta$ and the smooth test configuration $(\mathscr{M}, \mathscr{L})$ has a reduced central fibre, then $\mathscr{F}_{K}^{\operatorname{ext}}(\mathscr{M}, \mathscr{L}) \geq 0$. This leads to a notion of a relative weighted $\mathrm{K}$-semistabilty of $(M, J, L, \mathbb{T}, K)$ which obstructs the existence of $K$-extremal Kähler metrics in $2 \pi c_{1}(L)$.

Our first main result extends this weighted K-semistability to weighted K-stability.

Theorem 1 (see Theorem [5.5). Let $(N, \mathcal{D}, J, X)$ be a compact regular Sasaki manifold, and $(M, J, L)$ the corresponding smooth polarized Kähler manifold. Suppose that $K$ is a Sasaki-Reeb vector field on $(N, \mathcal{D}, J)$ which commutes with $X$, such that $N$ admits an extremal Sasaki structure compatible with the transversal holomorphic structure $\left(K, J^{K}\right)$ determined by $(K, \mathcal{D}, J)$. Let $\mathbb{T}$ be a maximal torus in the group Aut $(M, L)$ of automorphisms of the polarized variety $(M, J, L)$, such that $X, K$ belong to Lie( $\mathbb{T})$. Then, for any $\mathbb{T}$-equivariant smooth polarized test configuration $(\mathscr{M}, \mathscr{L})$ associated to $(M, J, L, \mathbb{T})$, which has reduced central fibre and is not a product test configuration, we have $\mathscr{F}_{K}^{\operatorname{ext}}(\mathscr{M}, \mathscr{L})>0$, i.e., $(M, J, L, K, \mathbb{T})$ is relative weighted $K$-stable on such test configurations.

By Proposition 1, the existence of an extremal Sasaki structure on $\left(N, K, J^{K}\right)$ translates to the existence of a $\mathbb{T}$-invariant $K$-extremal metric $(g, \omega)$ on $M$ with $\omega \in 2 \pi c_{1}(L)$. In 
this setting it is well-known (see in particular [8, 33, 52, for unweighted CSC and extremal Kähler metrics) that a K-stability result like Theorem 1 may be deduced by establishing a suitable properness property for an appropriate Mabuchi energy functional. Furthermore, there is an axiomatic framework [21] for establishing such properness properties, which allows us to focus on the specificities of our problem.

In our setting, we denote the relevant functional by $\mathbf{M}_{\check{K}, \kappa}$, where the subscripts $\check{K}$ and $\kappa$ stand respectively for the holomorphic vector field on $(M, J)$ induced by $K$, and a positive real constant, determined by $K$, see $\$ 3.3$. This functional is a weighted version of the relative Mabuchi energy introduced in [39] defined on the space of $\mathbb{T}$-invariant hermitian products on $L$ with positive curvature. To prove the relative weighted K-stability of $(M, J, L, K, \mathbb{T})$, it suffices show that $\mathbf{M}_{\check{K}, \kappa}$ is proper with respect to the natural action of the complexified torus $\mathbb{T}_{\mathbb{C}}$ on that space.

In order to apply the axiomatic framework of [21], we need to extend $\mathbf{M}_{\breve{K}, \kappa}$ to the $d_{1^{-}}$ completion of the space of $\mathbb{T}$-invariant relative Kähler potentials on $M$. The existence of such an extension is not obvious because, in contrast to the CSC Kähler case, the definition of $\mathbf{M}_{\breve{K}, \kappa}$ involves the $\kappa$-normalized potential of $\check{K}$ with respect to a metric $\omega \in c_{1}(L)$, which has no natural interpretation in the space of weakly regular relative Kähler potentials.

The key for bypassing this difficulty is also the key to the proof of Proposition 1. Namely, we make use of a bijection $\hat{\boldsymbol{\Theta}}$ (see $\$ 2.3$, and in particular equation (19)) between (suitably normalized) Sasaki structures on $\left(N, K, J^{K}\right)$ and $\left(N, X, J^{X}\right)$ such that corresponding Sasaki structures have the same underlying CR structure. This map comes from a correspondence between their respective spaces of (radial) Kähler potentials on the cone $(V, J, K)$ that we establish (see Corollary 2.15) building on the work of He-Sun [36]. While we avoid the noncompact cone in the notion of stability we use, $(V, J, K)$ thus plays an essential role in establishing properties of the map $\hat{\boldsymbol{\Theta}}$.

First, after identifying the normalized Sasaki structures on $\left(N, K, J^{K}\right)$ with $\mathbb{T}$-invariant hermitian forms on $L$ with positive curvature, we show (see Lemma 4.4) that

$$
\mathbf{M}_{\breve{K}, \kappa} \circ \hat{\boldsymbol{\Theta}}=\mathbf{M}^{K}
$$

$\mathbf{M}^{K}$ is the relative Mabuchi energy acting on the space of (normalized) Sasaki structures in $\left(N, K, J^{K}\right)$, studied in [34, 35, 56]. Secondly, in Lemma 2.17 we compute the derivative of $\hat{\boldsymbol{\Theta}}$, which enables us to show (see Lemma 4.6) that it is bilipschitz with respect to $d_{1}$. Thirdly, $\hat{\boldsymbol{\Theta}}$ is equivariant for the natural action of the complexified torus $\mathbb{T}_{\mathbb{C}}$ induced by $K$ and $X$ on the space of $\mathbb{T}$-invariant hermitian products on $L$ (similar to the setting in [30]). This is different than the action of complex automorphisms on the space of relative Kähler potentials considered in [8, 21, 33, 52, but has the practical advantage to induce also a natural action on the space of (normalized) Sasaki structures in $\left(N, K, J^{K}\right)$.

These tools allow us to define a notion of properness for $\mathbf{M}_{\check{K}, \kappa}$ with respect to the natural action of $\mathbb{T}_{\mathbb{C}}$ which directly translates via $\hat{\boldsymbol{\Theta}}$ to a corresponding notion of properness for $\mathbf{M}^{K}$ (see Corollary 4.11). This allows us to use the extension of $\mathbf{M}^{K}$ to the $d_{1}$-completion and the regularity of its minimizers (obtained in [34, 35]) to show the properness of $\mathbf{M}^{K}$ (see Theorem 4.12) and hence of $\mathbf{M}_{\check{K}, \kappa}$. A key result for this to work is the transitivity of the action of $\mathbb{T}_{\mathbb{C}}$ on the space of $\mathbb{T}$-invariant $K$-extremal Kähler metrics in $2 \pi c_{1}(L)$, which

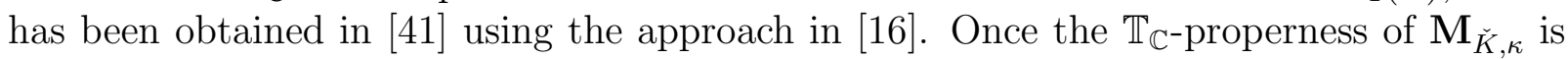
established, the proof of Theorem 1 essentially follows from the arguments in [8, 52].

Since the complex cone $(V, J, K)$ plays such an important role in the proof, we spend some time in Section 2 developing its properties, following [14, 18, 36, 47]. In particular, in $\$ 2.2$, we detail the relation (see [14, 18 and Lemma 2.13) between various known points of view on the Reeb cone $\mathfrak{t}_{+}$in the Lie algebra $\mathfrak{t}$ of $\mathbb{T}$, establishing that $\mathfrak{t}_{+}$consists of vector fields $K$ whose cones $(V, J, K)$ admit a radial Kähler potential. 
Weighted K-stability versus K-stability of affine cones. Theorem 1 provides in particular an obstruction to the existence of CSC Sasaki structures compatible with a transversal holomorphic structure $\left(K, J^{K}\right)$ on $N$, expressed in terms of the weighted $\mathrm{K}$ stability of a fixed regular quotient $(M, J, L, \mathbb{T})$ with respect to suitable test configurations $(\mathscr{M}, \mathscr{L})$. We first remark that the assumptions that $\mathscr{M}$ is smooth and its central fibre is reduced are needed in the proof of Theorem 1 (as in [40]) both to define $\mathscr{F}_{K}^{\operatorname{ext}}(\mathscr{M}, \mathscr{L})$ and to relate it to the slope of the corresponding Mabuchi energy $\mathbf{M}_{\check{K}, \kappa}$. However, it is shown in [23, 51] that in the CSC case, restricting to such test configurations of $(M, J, L)$ does not affect the corresponding (unweighted) K-stability condition. We expect that these assumptions on $(\mathscr{M}, \mathscr{L})$ will not limit the applicability of Theorem 1 either.

Secondly, we need to compare the weighted K-stability in Theorem 1 to the (relative) $\mathrm{K}$-stability of the corresponding affine cone $(V, J, K, \mathbb{T})$ in the sense of [18]. To do this, we use the fact that the complex manifold $(V, J)$ can be identified with $\left(L^{*}\right)^{\times}$(the total space of the dual bundle of $L$ with the zero section removed) whereas for any test configuration $(\mathscr{M}, \mathscr{L})$ as in Theorem 1, the corresponding complex cone $\mathscr{V}=\left(\mathscr{L}^{*}\right)^{\times}$gives rise to a $\mathbb{T}$-equivariant smooth test configuration of the polarized affine cone $(V, J, K)$ in the sense of [18, 45]. We shall refer to such test configurations of $(V, J, K)$ as smooth $\mathbb{T}$-equivariant regular test configurations (where we have also assumed that the central fibre of $\mathscr{M}$ is reduced). We then have the following result.

Theorem 2 (see Corollary 6.10). Let $(V, J, K)$ be the polarized complex cone associated to a compact Sasaki manifold $(N, \mathcal{D}, J, K), \mathbb{T} \leqslant$ Aut $(V, J)^{K}$ a maximal torus and assume that the Lie algebra of $\mathbb{T}$ contains a regular Sasaki-Reeb vector field $X$. If $(V, J, K)$ admits a scalar-flat Kähler cone metric, then for any $\mathbb{T}$-equivariant regular test configuration $(\mathscr{V}, K)$ of $(V, J, K)$, which is not obtained from a product test configuration, the Donaldson-Futaki invariant of [18] associated to $(\mathscr{V}, K)$ is strictly positive.

This theorem follows readily from Theorem 1 once we establish that the DonaldsonFutaki invariant of [18] associated to $\left(\mathscr{L}^{*}\right)^{\times}$is a positive multiple of the corresponding weighted Donaldson-Futaki invariant $\mathscr{F}_{K}(\mathscr{M}, \mathscr{L})$. To establish such a relationship, we propose in Definition 6.7 a differential geometric definition of a Donaldson-Futaki invariant for any smooth test configuration $(\mathscr{V}, K)$ (which itself is a complex affine cone) associated to the affine cone $(V, J, K, \mathbb{T})$ of $\left(N, K, J^{K}\right)$. We refer to this quantity as the global Futaki invariant and believe it is of independent interest. It is expressed as an integral formula on the Sasaki manifold $\left(\mathscr{N}, \mathscr{D}, \eta_{\mathscr{D}}^{K}\right)$ associated to $\mathscr{V}$ as

$$
\mathscr{G}_{\mathscr{F}}{ }_{K}(\mathscr{V})=\frac{c_{K}}{(m+1) !} \int_{\mathscr{N}} \eta_{\mathscr{D}}^{K} \wedge\left(d \eta_{\mathscr{D}}^{K}\right)^{m+1}+\frac{2}{m !} \int_{\mathscr{N}}\left(\pi^{*} \rho_{o}-\rho\right) \wedge \eta_{\mathcal{D}}^{K} \wedge\left(d \eta_{\mathcal{D}}^{K}\right)^{m},
$$

where $\pi: \mathscr{V} \rightarrow \mathbb{C} P^{1}$ is the equivariant map of the test configuration, $c_{K}$ a normalizing constant, $\rho$ is any representative of the basic first Chern class $2 \pi c_{1}^{B}$, and $\rho_{o} \in 2 \pi c_{1}\left(\mathbb{C} P^{1}\right)$. We justify this definition in Proposition 6.8 by proving that the Donaldson-Futaki invariant [18] of any $\mathbb{T}$-equivariant regular test configuration $\mathscr{V}$ for $(V, J, K)$ is a constant positive multiple of its global Futaki invariant. Then in Proposition 6.9, we prove that, up to a factor of $2 \pi$, the weighted Futaki invariant of $\mathbb{T}$-equivariant smooth polarized test configuration $(\mathscr{M}, \mathscr{L})$ for $(M, J, L, \mathbb{T})$ agrees with the global Futaki invariant of its cone $\mathscr{V}$.

Another interesting aspect of our approach is that it allows for the consideration (as in [23, 40, 51]) of Kähler $\mathbb{T}$-equivariant smooth test configurations $(\mathscr{M}, \mathscr{A})$ associated to the regular quotient $(M, J, L)$, i.e., allowing both non-projective total spaces $\mathscr{M}$ and transcendental Kähler classes $\mathscr{A}$ on $\mathscr{M}$. Notice that in this more general framework, the weighted $\mathrm{K}$-semistability of $(M, J, L, K, \mathbb{T})$ still holds by the results in [40]. The novel 
implication of this to the theory of extremal Sasaki structures is that a smooth Kähler $\mathbb{T}$ equivariant test configuration $(\mathscr{M}, \mathscr{A})$ for which $\mathscr{A}$ is not an integral class does not define a test configuration of the polarized corresponding affine cone $(V, J, K)$. This suggests (see Conjecture 5.8) that Theorem 1 extends to $\mathbb{T}$-equivariant smooth Kähler test configurations $(\mathscr{M}, \mathscr{A})$ associated to $(M, J, L, \mathbb{T})$.

A Yau-Tian-Donaldson correspondence on admissible Sasaki manifolds. The importance of extending the K-stability criterion to Kähler test configurations goes back to an example from [4] which suggests that polarized test configurations are in general insufficient to detect the non-existence of extremal Kähler metrics on $(M, J, L)$. In the final section of the article, we obtain Sasaki analogues of some results in [4]. To this end, we let $(g, J, \omega)$ be a Kähler metric on the total space $M$ of a $\mathbb{P}^{1}$-bundle over the product of CSC polarized Kähler manifolds, obtained by the Calabi construction (also called admissible), and endow $M$ with a fibrewise $\mathbb{S}^{1}$-action which gives rise to a Killing vector field $\check{K}$ on $(M, J, \omega)$. Furthermore, assuming also that $\omega \in 2 \pi c_{1}(L)$ for a polarization $L$ on $M$, we let $K$ be the vector field on $L$ defined by $\check{K}$ and a positive Killing potential for $\check{K}$. In this setting, the search for $K$-extremal Kähler metrics in $\zeta$ given by the Calabi ansatz has been recently studied in [6]. The authors show there that (similarly to the extremal case studied in [4]), there exists a polynomial $P(z)$ of degree $\leq m+2$ which is positive on the interval $(-1,1)$ if and only if $(M, J)$ admits a $K$-extremal Kähler metric in $\zeta$, and in this case, the extremal Kähler metric is given by the Calabi ansatz. On the other hand, the computations from [6, 40] also show that for each $z_{0} \in(-1,1), P\left(z_{0}\right)$ computes (up to a positive multiple) the relative weighted Donaldson-Futaki invariant of a smooth Kähler test configuration $\left(\mathscr{M}, \mathscr{A}_{z_{0}}\right)$ associated to $(M, J, \zeta)$; furthermore, $\left(\mathscr{M}, \mathscr{A}_{z_{0}}\right)$ is a polarized test configuration precisely when $z_{0} \in(-1,1) \cap \mathbb{Q}$. Thus, the weighted K-stability established in Theorem 1 only yields that $P(z)$ must be positive on $(-1,1) \cap \mathbb{Q}$ should a $K$-extremal metric exist, whereas Conjecture 5.8 mentioned above would imply the positivity of $P(z)$ everywhere on $(-1,1)$, i.e., the existence of a $K$-extremal metric of Calabi type. Therefore the following result provides evidence for Conjecture 5.8 .

Theorem 3 (see Corollary [7.3). Let $(N, \mathcal{D}, J, X)$ be the regular Sasaki manifold corresponding to an admissible (polarized) Kähler manifold $(M, J, \omega)$ and $K$ be a Sasaki-Reeb vector field corresponding to a lift (which need not be quasiregular) of the generator of fibrewise $\mathbb{S}^{1}$-action on $M$. Then $N$ admits an extremal Sasaki structure in $\left(K, J^{K}\right)$ if and only if the corresponding polynomial $P(z)$ is positive on $(-1,1)$. In this case, the extremal Sasaki structure corresponds to an K-extremal Kähler metric on $(M, J, \zeta)$ of Calabi type.

We notice that, as in 44, Theorem 3 provides examples of Sasaki manifolds $\left(N, K, J^{K}\right)$ (obtained as $\mathbb{S}^{1}$-bundles over admissible manifolds), which do not admit extremal Sasaki structures for the reason that the corresponding polynomial $P(z)$ has a unique double irrational root in $(-1,1)$. Our theory shows that one way to detect the non-existence in this case is to consider Kähler test configurations on the regular quotient.

\section{Preliminaries on Sasaki geometry}

1.1. Sasaki structures and transversal Kähler structures. Let $(N, \mathcal{D})$ be a contact $(2 m+1)$-manifold and denote the Levi form of $\mathcal{D}$ by $L_{\mathcal{D}}: \mathcal{D} \times \mathcal{D} \rightarrow T N / \mathcal{D}$; the latter is defined, via local sections $X, Y \in C_{N}^{\infty}(\mathcal{D})$, by the tensorial expression $L_{\mathcal{D}}(X, Y)=$ $-\eta_{\mathcal{D}}([X, Y])$, where $\eta_{\mathcal{D}}: T N \rightarrow T N / \mathcal{D}$ is the quotient map. A contact vector field is a vector field $X$ on $N$ such that $\mathcal{L}_{X}\left(C_{N}^{\infty}(\mathcal{D})\right) \subseteq C_{N}^{\infty}(\mathcal{D})$. We denote by $\operatorname{con}(N, \mathcal{D})$ the Lie algebra of contact vector fields in $C_{N}^{\infty}(T N)$.

We recall the following basic fact in the theory of contact manifolds (see e.g. [1]]). 
Lemma 1.1. The map $X \mapsto \eta_{\mathcal{D}}(X)$ from contact vector fields to sections of $T N / \mathcal{D}$ is a linear isomorphism, whose inverse $\sigma \mapsto X_{\sigma}$ is a first order linear differential operator.

In particular, for any vector field $X$ on $N$, there is a unique contact vector field $X_{\tau}$ (namely the one with $\tau=\eta_{\mathcal{D}}(X)$ ) such that $X-X_{\tau}$ is a section of $\mathcal{D}$.

Now suppose $J \in \operatorname{End}(\mathcal{D})$ is a $\mathrm{CR}$ structure on $(N, \mathcal{D})$, i.e., a (fibrewise) complex structure on $\mathcal{D}$ such that $\mathcal{D}^{(1,0)}:=\{X-i J X \mid X \in \mathcal{D}\}$ is closed under Lie bracket in $T N \otimes \mathbb{C}$; then we denote by

$$
\mathfrak{c r}(N, \mathcal{D}, J):=\left\{X \in \mathfrak{c o n}(N, \mathcal{D}) \mid \mathcal{L}_{X} J=0\right\}
$$

the Lie subalgebra of $\mathfrak{c o n}(N, \mathcal{D})$, whose elements correspond to CR vector fields $X$ on $(N, \mathcal{D}, J)$. If moreover $(\mathcal{D}, J)$ is strictly pseudoconvex then $T N / \mathcal{D}$ has a canonical orientation (and so $(N, \mathcal{D})$ is co-oriented): the positive sections $\tau$ are those for which $\tau^{-1} L_{\mathcal{D}}(\cdot, J \cdot)$ is positive definite. Note that $\tau^{-1} L_{\mathcal{D}}=\left.d \eta_{\mathcal{D}}^{X}\right|_{\mathcal{D}}$ where $\eta_{\mathcal{D}}^{X}:=\tau^{-1} \eta_{\mathcal{D}}$ is the contact form defined by $\tau=\eta_{\mathcal{D}}(X)$. We let $\mathfrak{c o n}_{+}(N, \mathcal{D})$ denote the space Reeb vector fields of $(N, \mathcal{D})$ for which $\eta_{\mathcal{D}}(X)$ is positive with respect to the chosen orientation. We then have the following fundamental definitions (see e.g. [12]).

Definition 1.2. Let $(N, \mathcal{D}, J)$ be a strictly pseudoconvex CR manifold. Then the SasakiReeb cone of $(N, \mathcal{D}, J)$ is $\mathfrak{c r}_{+}(N, \mathcal{D}, J):=\mathfrak{c r}(N, \mathcal{D}, J) \cap \mathfrak{c o n}_{+}(N, \mathcal{D})$. If $\mathfrak{c r}_{+}(N, \mathcal{D}, J)$ is nonempty then $(N, \mathcal{D}, J)$ is said to be of Sasaki type, an element $X \in \mathfrak{c r}_{+}(N, \mathcal{D}, J)$ is called Sasaki-Reeb vector field or a Sasaki structure on $(N, \mathcal{D}, J)$, and $(N, \mathcal{D}, J, X)$ is called a Sasaki manifold. We say $X$ is quasiregular if the flow of $X$ generates an $\mathbb{S}^{1}$ action on $N$, and moreover regular if this action is free.

The following well-known construction provides a standard way (see e.g. [11]) to extend geometric notions on Kähler manifolds to Sasaki manifolds.

Example 1.3. Let $(M, J, g, \omega)$ be a Kähler manifold such that $[\omega / 2 \pi]$ is an integral de Rham class. Then there is a principal $\mathbb{S}^{1}$-bundle $\pi: N \rightarrow M$ with a connection 1 -form $\eta$ satisfying $d \eta=\pi^{*} \omega$. Thus $(N, \mathcal{D}, J, X)$ is a Sasaki manifold, where $\mathcal{D}=\operatorname{ker} \eta \subseteq T N, J$ is the pullback of the complex structure on $T M$ to $\mathcal{D} \cong \pi^{*} T M$ and $X$ is the generator of the $\mathbb{S}^{1}$ action (with $\eta(X)=1$, so $\eta=\eta_{\mathcal{D}}^{X}$ ).

Conversely, if $X \in \mathfrak{c r}_{+}(N, \mathcal{D}, J)$ is (quasi)regular Sasaki-Reeb vector field on $(N, \mathcal{D}, J)$, then $N$ is a principal $\mathbb{S}^{1}$-bundle (or orbibundle) $\pi: N \rightarrow M$ over a Kähler manifold (or orbifold) $M$. Irrespective of regularity, this correspondence between Kähler geometry and Sasaki geometry holds locally: any point of a Sasaki manifold $(N, \mathcal{D}, J, X)$ has a neighbourhood in which the leaf space $M$ of the flow of $X$ is a manifold and has a Kähler structure $(g, J, \omega)$ induced, using the local identification of $\mathcal{D}$ and $\pi^{*} T M$, by the transversal Kähler structure $\left(g_{X}, J, \omega_{X}\right)$ on $\mathcal{D}$, where $\omega_{X}:=\left.d \eta_{\mathcal{D}}^{X}\right|_{\mathcal{D}}$ and $g_{X}:=\omega_{X}(\cdot, J \cdot)$. Indeed $g_{X}, J$, and $\omega_{X}$ are all $X$-invariant, so they all descend to $M$, and we refer to $(M, g, J, \omega)$ as a Sasaki-Reeb quotient of $(N, \mathcal{D}, J, X)$.

For $X \in \mathfrak{c r}(N, \mathcal{D}, J)$ we set

$$
\begin{aligned}
& \mathfrak{c o n}(N, \mathcal{D}, J)^{X}:=\{K \in \mathfrak{c o n}(N, \mathcal{D}) \mid[X, K]=0\}, \\
& \mathfrak{c r}(N, \mathcal{D}, J)^{X}:=\mathfrak{c o n}(N, \mathcal{D}, J)^{X} \cap \mathfrak{c r}(N, \mathcal{D}, J), \\
& \mathfrak{c r}_{+}(N, \mathcal{D}, J)^{X}:=\mathfrak{c o n}(N, \mathcal{D}, J)^{X} \cap \mathfrak{c r} \mathfrak{r}_{+}(N, \mathcal{D}, J) .
\end{aligned}
$$

If in addition $X \in \mathfrak{c r}_{+}(N, \mathcal{D}, J)$ then

$$
C_{N}^{\infty}(\mathbb{R})^{X}:=\left\{f \in C_{N}^{\infty}(\mathbb{R}): d f(X)=0\right\}
$$

is a Lie algebra under the transversal Poisson bracket $\left\{f_{1}, f_{2}\right\}:=-\omega_{X}^{-1}\left(\left.d f_{1}\right|_{\mathcal{D}},\left.d f_{2}\right|_{\mathcal{D}}\right)$, and we have the following elementary but central lemma (see [1]). 
Lemma 1.4. The map $K \mapsto f=\eta_{\mathcal{D}}^{X}(K)$ is a Lie algebra isomorphism from $\operatorname{con}(N, \mathcal{D}, J)^{X}$ to $C_{N}^{\infty}(\mathbb{R})^{X}$, and $K \in \mathfrak{c r}(N, \mathcal{D}, J)^{X}$ if and only if $f$ is a transversal Killing potential for $\left(g_{X}, \omega_{X}\right)$, i.e., $-\omega_{X}^{-1}(d f)$ is a transversal Killing vector field for $g_{X}$.

1.2. Transversal holomorphic structures. Let $N$ be a connected $(2 m+1)$-manifold, let $X$ be a nowhere zero vector field, and let

$$
\beta_{X}: T N \rightarrow \mathcal{D}_{X}:=T N / \mathbb{R} \cdot X
$$

be the quotient of $T N$ by the span of $X$. Thus $\mathcal{D}_{X}$ is everywhere locally canonically isomorphic to the pullback of the tangent bundle of local quotients of $N$ by $X$.

Definition 1.5. A transversal holomorphic structure on $(N, X)$ is a complex structure $J^{X}$ on $\mathcal{D}_{X}$ which is everywhere locally the pullback of a complex structure (i.e., an integrable almost complex structure) on a local quotient of $N$ by $X$.

Any Sasaki structure $(\mathcal{D}, J, X)$ on $N$ induces a transversal holomorphic structure on $(N, X)$ : since $\mathcal{D}$ is transverse to $X,\left.\beta_{X}\right|_{\mathcal{D}}$ is a bundle isomorphism $\mathcal{D} \rightarrow \mathcal{D}_{X}$, and the complex structure on $\mathcal{D}_{X}$ induced by $J$ is $X$-invariant and integrable because $J$ is.

Definition 1.6. [12] A transversal holomorphic structure $J^{X}$ on $(N, X)$ has Sasaki type if $N$ admits a Sasaki structure $(\mathcal{D}, J, X)$ which is compatible with $\left(X, J^{X}\right)$, i.e.,

$$
\left.\beta_{X}\right|_{\mathcal{D}}: \mathcal{D} \rightarrow \mathcal{D}_{X} \text { intertwines } J \text { and } J^{X} \text {. }
$$

Such compatible Sasaki structures on $\left(N, X, J^{X}\right)$ are completely determined by their contact forms or, equivalently, by the corresponding contact distributions: we let $\mathcal{S}\left(X, J^{X}\right) \leqslant$ $\Omega^{1}(N)$ be the subspace of contact forms of Sasaki structures compatible with $\left(X, J^{X}\right)$, and write $\eta_{\mathcal{D}}^{X}$ for the unique element of $\mathcal{S}\left(X, J^{X}\right)$ with $\operatorname{ker} \eta_{\mathcal{D}}^{X}=\mathcal{D}$.

As is well-known, the geometry of local quotients of $N$ may be described using the basic de Rham complex

$$
\Omega_{X}^{\bullet}(N)=\left\{\alpha \in \Omega^{\bullet}(N): \imath_{X} \alpha=0=\mathcal{L}_{X} \alpha\right\}
$$

with differential $d_{X}$ given by restriction of $d$ (which preserves basic forms); note that $\Omega_{X}^{0}(N)=C_{N}^{\infty}(\mathbb{R})^{X}$ —we also denote the closed and exact forms in $\Omega_{X}^{\bullet}(N)$ by $\Omega_{X, \mathrm{cl}}^{\bullet}(N)$ and $\Omega_{X, \text { ex }}^{\bullet}(N)$ respectively. Any vector field $Y$ with $\mathcal{L}_{X} Y=f X$ is projectable onto local quotients, and hence induces Lie derivatives on both basic forms and $X$-invariant sections of $\mathcal{D}_{X}$ (i.e., $Z \in C_{N}^{\infty}\left(\mathcal{D}_{X}\right)$ with $\mathcal{L}_{X} Z=0$ ); these Lie derivatives depend on $Y$ only via the $X$-invariant section $\beta_{X} Y$ of $\mathcal{D}_{X}$, hence may be denoted $\mathcal{L}_{\beta_{X} Y}$.

A complex structure $J^{X}$ on $\mathcal{D}_{X}$ is thus a transversal holomorphic structure if and only if $\mathcal{L}_{X} J^{X}=0$ and for any $X$-invariant section $Z$ of $\mathcal{D}, \mathcal{L}_{J^{X}} J^{X}=J^{X} \circ \mathcal{L}_{Z} J^{X}$. Note that $\left\{\alpha \in \wedge^{k} T^{*} N \mid \imath_{X} \alpha=0\right\}$ is naturally isomorphic to $\wedge^{k} \mathcal{D}_{X}^{*}$, so the $X$-invariance of $J^{X}$ implies that it induces an operator on $\Omega_{X}^{\bullet}(N)$, as in complex geometry. We thus obtain a twisted exterior derivative $d_{X}^{c}=J^{X} \circ d_{X} \circ\left(J^{X}\right)^{-1}$ on $\Omega_{X}^{\bullet}(N)$. Also, the integrability of $J^{X}$ means equivalently that the basic $(1,0)$-forms generate a differential ideal.

If $\left(N, X, J^{X}\right)$ is compact of Sasaki type, then $\Omega_{X}^{\bullet}(N)$ has a Hodge decomposition with respect to the induced transversal metric of any $\eta \in \mathcal{S}\left(X, J^{X}\right)$, and the $d_{X} d_{X}^{c}$-lemma and transversal Kähler identities are satisfied [26], with the following consequences, cf. [11, 12].

Lemma 1.7. A 1-form $\gamma$ on $N$ is basic with $J^{X}$-invariant exterior derivative if and only if $\gamma=d_{X}^{c} \varphi+\alpha$ for a basic function $\varphi \in \Omega_{X}^{0}(N)$ and a closed basic 1-form $\alpha \in \Omega_{X, c l}^{1}(N)$; further, $\alpha$ is uniquely determined by $\gamma$, as is $\varphi$ up to an additive constant. In particular:

(i) $\mathcal{S}\left(X, J^{X}\right)$ is an open subset of an affine space with translation group $\Omega_{X}^{0}(N) / \mathbb{R} \times$ $\Omega_{X, \mathrm{cl}}^{1}(N)$, where $\Omega_{X}^{0}(N) / \mathbb{R}$ denotes the quotient of $\Omega_{X}^{0}(N)$ by constants; furthermore this open subset is $\Omega_{X, \mathrm{cl}}^{1}(N)$-invariant and convex. 
(ii) if $Y$ is a projectable vector field, and $\eta \in \mathcal{S}\left(X, J^{X}\right)$, then $\mathcal{L}_{Y} \eta$ is tangent to $\mathcal{S}\left(X, J^{X}\right)$ (at $\eta$ ) if and only if

$$
Y=J X_{\varphi}+Y^{H}+X_{f}+h X
$$

where $X_{\varphi}$ and $X_{f}$ are contact vector fields with respect to $\eta, J$ is the CR structure induced by $\eta$ with $J X=0, \varphi, f$ and $h$ are basic functions uniquely defined (by $Y$ and $\eta$ ) up to $\varphi \mapsto \varphi+b, f \mapsto f+c$ and $h \mapsto h-c$ for constants $b, c \in \mathbb{R}$, and $\alpha^{H}=\iota_{Y^{H}} d \eta$ is a uniquely defined basic harmonic 1-form with respect to the transversal metric induced by $\eta$. Then $\mathcal{L}_{Y} \eta=d_{X}^{c} \varphi+\alpha^{H}+d h$, so any tangent vector to $\mathcal{S}\left(X, J^{X}\right)$ arises in this way.

Proof. If $\gamma=d_{X}^{c} \varphi+\alpha$ (as stated), it is basic with $d_{X} \gamma=d_{X} d_{X}^{c} \varphi$, which is $J^{X}$-invariant because $J^{X} d_{X} d_{X}^{c} \varphi=-d_{X}^{c} d_{X} \varphi=d_{X} d_{X}^{c} \varphi$. Conversely if $d_{X} \gamma$ is $J^{X}$-invariant, $\gamma$ is $d_{X} d_{X^{-}}^{c}$ closed, so $\gamma=d_{X}^{c} \varphi+\alpha$ (as stated) by the $d_{X} d_{X}^{c}$-lemma.

(i) Note that $\eta \in \Omega^{1}(N)$ is in $\mathcal{S}\left(X, J^{X}\right)$ if and only if $\eta(X)=1, \mathcal{L}_{X} \eta=0$ and $d \eta \in \Omega_{X}^{2}(N)$ is $J^{X}$-invariant and $J^{X}$-positive. Thus if also $\eta_{0} \in \mathcal{S}\left(X, J^{X}\right), \eta-\eta_{0}$ is basic with $J^{X_{-}}$ invariant exterior derivative, hence

$$
\eta=\eta_{0}+d_{X}^{c} \varphi+\alpha
$$

with $\varphi \in \Omega_{X}^{0}(N)$ and $\alpha \in \Omega_{X, \mathrm{cl}}^{1}(N)$. Conversely, given $\eta_{0} \in \mathcal{S}\left(X, J^{X}\right)$, such an $\eta$ is in $\mathcal{S}\left(X, J^{X}\right)$ if $d \eta$ is $J^{X}$-positive - a convex open condition depending only on $\eta \bmod \Omega_{X, \mathrm{cl}}^{1}(N)$.

(ii) For any basic function $h, \mathcal{L}_{h X} \eta=d(\eta(h X))=d h$, which is tangent to $\mathcal{S}\left(X, J^{X}\right)$. Hence if $\mathcal{L}_{Y} \eta$ is tangent to $\mathcal{S}\left(X, J^{X}\right)$, then $Y=Y_{0}+\eta(Y) X$ with $\eta\left(Y_{0}\right)=0$ and $\mathcal{L}_{Y_{0}} \eta=$ $\iota_{Y_{0}} d \eta$ tangent to $\mathcal{S}\left(X, J^{X}\right)$, i.e., of the form $d^{c} \varphi+\alpha_{0}$. We write $\alpha_{0}=\alpha^{H}-d f$ with $f$ a basic function and $\alpha^{H}$ a basic harmonic form with respect to the transversal metric induced by $\eta$. Thus $Y_{0}=J X_{\varphi}+Y^{H}+X_{f}-f X$ with $\iota_{Y^{H}} d \eta=\alpha^{H}$, and $Y$ has the stated form with $h=\eta(Y)-f$. Clearly any such $Y$ has

$$
\mathcal{L}_{Y} \eta=d \iota_{Y} \eta+\iota_{Y} d \eta=d(f+h)+d^{c} \varphi+\alpha^{H}-d f=d^{c} \varphi+\alpha^{H}+d h,
$$

which is tangent to $\mathcal{S}\left(X, J^{X}\right)$, and the uniqueness claims follow straightforwardly using the Hodge decomposition.

Remark 1.8. Lemma 1.7(i) shows that $\mathcal{S}\left(X, J^{X}\right)$ is connected. It follows that for any $\eta, \eta_{0} \in \mathcal{S}\left(X, J^{X}\right)$, the Gray-Moser theorem implies that there exists $\Phi \in \operatorname{Diff}_{0}(N)$, the identity component of the diffeomorphism group of $N$, with $\Phi^{*} \eta=\eta_{0}$. Lemma 1.7(ii) is an infinitesimal version of this fact: any element of $T_{\eta} \mathcal{S}\left(X, J^{X}\right)$ is of the form $\mathcal{L}_{Y} \eta$ with $Y$ as in (11) uniquely determined up to a contact vector field of $\mathcal{D}=\operatorname{ker} \eta$. By Lemma 1.1 we can fix the choice of $Y$ with $\mathcal{L}_{Y} \eta=d^{c} \varphi+\alpha^{H}+d h$ by subtracting the contact vector field $X_{\eta(Y)}=X_{f+h}$ to obtain a section

$$
Z=J X_{\varphi}+Y^{H}-X_{h}+h X
$$

of $\mathcal{D}=\operatorname{ker} \eta$. This yields a bijection between $T_{\eta} \mathcal{S}\left(X, J^{X}\right)$ and sections $Z$ of $\mathcal{D}$ such that the basic 2 -form $d \mathcal{L}_{Z} \eta=\mathcal{L}_{Z} d \eta$ is $J^{X}$-invariant.

Definition 1.9. The diffeomorphism group $\operatorname{Diff}(N)$ acts naturally on pairs $\left(X, J^{X}\right)$, and we define the automorphism group $\operatorname{Aut}\left(N, X, J^{X}\right)$ to be the stabilizer of $\left(X, J^{X}\right)$; its formal Lie algebra $\mathfrak{a u t}\left(N, X, J^{X}\right)$ consists of $X$-invariant vector fields $Y$ with $\mathcal{L}_{\beta_{X} Y} J^{X}=0$.

The automorphism group is infinite dimensional: its formal Lie algebra contains an infinite dimensional abelian ideal $\mathfrak{o}(N, X)$ of $X$-invariant vector fields in the span of $X$; however, the quotient $\mathfrak{h}_{0}\left(N, X, J^{X}\right):=\mathfrak{a} \mathfrak{u} \mathfrak{t}\left(N, X, J^{X}\right) / \mathfrak{o}(N, X)$ may be identified with the space of holomorphic sections of $\mathcal{D}_{X}$ and hence is a finite dimensional complex Lie algebra if $N$ is compact [12. In this case, we let $\mathcal{O}(N, X)$ denote the abelian normal subgroup of $\operatorname{Aut}\left(N, X, J^{X}\right)$ generated by the exponential image of $\mathfrak{o}(N, X)$. However, the quotient 
group need not be well behaved: for example if $\mathbb{T}$ is a torus in $\operatorname{Aut}\left(N, X, J^{X}\right)$ containing $X$, then $\mathcal{O}(N, X) \cap \mathbb{T}$ is the group generated by $X$, which is not closed if $X$ is irregular.

By construction, $\operatorname{Aut}\left(N, X, J^{X}\right)$ acts on $\mathcal{S}\left(X, J^{X}\right)$ and hence elements $Y \in \mathfrak{a u t}\left(N, X, J^{X}\right)$ have the form (1) with respect to any $\eta \in \mathcal{S}\left(X, J^{X}\right)$ and induce vector fields $\eta \mapsto \mathcal{L}_{Y} \eta$ on $\mathcal{S}\left(X, J^{X}\right)$. In these terms, elements of $\mathfrak{h}_{0}\left(N, X, J^{X}\right)$ have representatives in $\mathcal{D}$ of the form $Y=J X_{\varphi}+Y^{H}+X_{f}-f X$, and the complex structure on $\mathfrak{h}_{0}\left(N, X, J^{X}\right)$ (coming from the action of $J^{X}$ on sections of $\left.\mathcal{D}_{X}\right)$ sends such a representative to $J X_{f}+J Y^{H}-X_{\varphi}+\varphi X$.

Remark 1.10. An element $h X$ of $\mathfrak{o}(N, X)$ induces the constant vector field $\eta \mapsto d h$ on $\mathcal{S}\left(X, J^{X}\right)$ and so if $N$ is compact, elements of $\mathcal{S}\left(X, J^{X}\right)$ related by an exact basic 1form $d h \in \Omega_{X, \text { ex }}^{1}(N)$ induce isomorphic Sasaki structures via the action of $\mathcal{O}(N, X)$, and $\mathcal{S}\left(X, J^{X}\right) / \mathcal{O}(N, X)=\mathcal{S}\left(X, J^{X}\right) / \Omega_{X, \text { ex }}^{1}(N)$.

On the other hand, elements of $\mathcal{S}\left(X, J^{X}\right)$ related by a closed basic 1-form induce the same transversal Kähler geometry (of local quotients): the transversal Kähler form $d \eta \in \Omega_{X}^{2}(N)$ depends only on $\eta+\Omega_{X, \mathrm{cl}}^{1}(N) \in \mathcal{S}\left(X, J^{X}\right) / \Omega_{X, \mathrm{cl}}^{1}(N)$. Since any two such forms are related by $d_{X} d_{X}^{c} \varphi$ for $\varphi \in \Omega_{X}^{0}(N)$, the quotient $\mathcal{S}\left(X, J^{X}\right) / \Omega_{X, \mathrm{cl}}^{1}(N)$ may be viewed as the transversal Kähler class of $\left(N, X, J^{X}\right)$.

This paper is concerned primarily with notions that depend only on the transversal Kähler geometry (of local quotients) and so it is desirable to fix the irrelevant translational gauge freedom by $\Omega_{X, \mathrm{cl}}^{1}(N)$, and hence identify the transversal Kähler class with a subspace $\Xi$ of $\mathcal{S}\left(X, J^{X}\right)$. This prompts the following definition.

Definition 1.11. A marking of a transversal holomorphic manifold $\left(N, X, J^{X}\right)$ of Sasaki type is an affine slice $\Xi \subseteq \mathcal{S}\left(X, J^{X}\right)$ to the action of $\Omega_{X, \mathrm{cl}}^{1}(N)$, i.e., a subspace of the form

$$
\Xi=\Xi\left(X, J^{X}, \eta_{0}\right):=\left\{\eta_{\varphi} \in \mathcal{S}\left(X, J^{X}\right) \mid \varphi \in \Omega_{X}^{0}(N)\right\}
$$

for some basepoint $\eta_{0} \in \mathcal{S}\left(X, J^{X}\right)$, where $\eta_{\varphi}:=\eta_{0}+d_{X}^{c} \varphi$. We then say $\left(N, X, J^{X}, \Xi\right)$ is marked. Elements of

$$
\hat{\Xi}\left(X, J^{X}, \eta_{0}\right):=\left\{\varphi \in \Omega_{X}^{0}(N) \mid \eta_{\varphi} \in \Xi\left(X, J^{X}, \eta_{0}\right)\right\}
$$

will be called Sasaki potentials with respect to $\eta_{0}$.

For any marking $\Xi$, we have $\Xi=\Xi\left(X, J^{X}, \eta_{0}\right)$ for any $\eta_{0} \in \Xi$. However, analogously to Kähler potentials, the space $\hat{\Xi}\left(X, J^{X}, \eta_{0}\right)$ of Sasaki potentials (which is an extension of $\Xi$ by $\mathbb{R}$ since $N$ is connected) depends upon the choice of basepoint $\eta_{0}$.

Remark 1.12. The automorphism group $\operatorname{Aut}\left(N, X, J^{X}\right)$ does not preserve a marking $\Xi$. A more invariant object is the image of $\Xi$ in $\mathcal{S}\left(X, J^{X}\right) / \Omega_{X \text {,ex }}^{1}(N)$ : the fibres of the projection of $\mathcal{S}\left(X, J^{X}\right) / \Omega_{X \text {,ex }}^{1}(N)$ to the transversal Kähler class $\mathcal{S}\left(X, J^{X}\right) / \Omega_{X, \mathrm{cl}}^{1}(N)$ are affine spaces modelled on the basic de Rham cohomology group $H_{X}^{1}(N, \mathbb{R})$, and the identity component $\operatorname{Aut}_{0}\left(N, X, J^{X}\right)$ acts trivially on basic de Rham cohomology, hence it preserves the image of the marking.

1.3. The Reeb cone of a transversal torus. Suppose $N$ is compact with transversal holomorphic structure $\left(X, J^{X}\right)$; then the automorphism group of any compatible Sasaki structure is compact. Hence if $\left(X, J^{X}\right)$ has Sasaki type, there is a torus $\mathbb{T} \leqslant \operatorname{Aut}\left(N, X, J^{X}\right)$ whose Lie algebra $\mathfrak{t}$ contains $X$, and we now fix such a $\mathbb{T}$. The considerations of the previous subsection apply equally to the space $\Omega_{X}^{\bullet}(N)^{\mathbb{T}}$ of $\mathbb{T}$-invariant basic forms, and the space $\mathcal{S}\left(X, J^{X}\right)^{\mathbb{T}}$ of $\mathbb{T}$-invariant elements of $\mathcal{S}\left(X, J^{X}\right)$. This space is acted upon by $\mathbb{T}$-equivariant automorphisms, i.e., by the centralizer $\operatorname{Aut}\left(N, X, J^{X}\right)^{\mathbb{T}}$ of $\mathbb{T}$ in $\operatorname{Aut}\left(N, X, J^{X}\right)$. Also, for any basepoint $\eta_{0} \in \mathcal{S}\left(X, J^{X}\right)^{\mathbb{T}}$, we define

$$
\Xi\left(X, J^{X}, \eta_{0}\right)^{\mathbb{T}}=\Xi\left(X, J^{X}, \eta_{0}\right) \cap \mathcal{S}\left(X, J^{X}\right)^{\mathbb{T}}
$$


and a corresponding space $\hat{\Xi}\left(X, J^{X}, \eta_{0}\right)^{\mathbb{T}}=\hat{\Xi}\left(X, J^{X}, \eta_{0}\right) \cap C_{N}^{\infty}(\mathbb{R})^{\mathbb{T}}$ of $\mathbb{T}$-invariant Sasaki potentials with respect to $\eta_{0}$. We refer to a subspace of $\mathcal{S}\left(X, J^{X}\right)^{\mathbb{T}}$ of the form (5) as a (T-invariant) marking, with the $\mathbb{T}$-invariance generally being understood from the context.

Any $K \in \mathfrak{t}$ is a $\mathrm{CR}$ vector field for the CR structure induced by any $\eta \in \mathcal{S}\left(X, J^{X}\right)^{\mathbb{T}}$. Furthermore, we have the following observation.

Lemma 1.13. Suppose that $N$ is compact and $K \in \mathfrak{t}$ is a Sasaki-Reeb vector field with respect to the $C R$ structure induced by some $\eta_{0} \in \mathcal{S}\left(X, J^{X}\right)^{\mathbb{T}}$. Then $K$ is a Sasaki-Reeb vector field with respect to the $C R$ structure induced by any $\eta \in \mathcal{S}\left(X, J^{X}\right)^{\mathbb{T}}$.

Proof. By assumption $\eta_{0}(K)>0$ on $N$, and we need to show that $f:=\eta(K)>0$. As $K$ is contact with respect to $\mathcal{D}:=$ ker $\eta$, we have $K=f X-\left(\left.d \eta\right|_{\mathcal{D}}\right)^{-1}\left(\left.d f\right|_{\mathcal{D}}\right)$ and hence

$$
\eta_{0}(K)=f-\left(\left.d \eta\right|_{\mathcal{D}}\right)^{-1}\left(\left.d f\right|_{\mathcal{D}},\left.\eta_{0}\right|_{\mathcal{D}}\right)
$$

Evaluating this equation at a global minimum $p$ of $f$ we obtain $f(p)=\eta_{0}(K)(p)>0$.

Definition 1.14. The Reeb cone of $\left(X, J^{X}, \mathbb{T}\right)$ is the cone [47]

$$
\mathfrak{t}_{+}^{X}:=\{K \in \mathfrak{t} \mid \eta(K)>0\}
$$

in $\mathfrak{t}$ determined by some (and hence any) $\eta \in \mathcal{S}\left(X, J^{X}\right)^{\mathbb{T}}$. Given any such $\eta$, its contact momentum map $\mu_{\eta}: N \rightarrow \mathfrak{t}^{*}$ is defined [44] by $\left\langle\mu_{\eta}, K\right\rangle=\eta(K)$.

Since $\left\langle\mu_{\eta}, X\right\rangle \equiv 1$, the image of $\mu_{\eta}$ in $\mathfrak{t}^{*}$ does not contain 0 . Furthermore, if $N$ is compact, results of [27, 44] therefore imply that $\Sigma:=\mathbb{R}^{+} \cdot \mu_{\eta}(N) \subseteq \mathfrak{t}^{*}$ is a strictly convex polyhedral cone (with the vertex $0 \in \mathfrak{t}^{*}$ removed) called the momentum cone of $\eta$. Moreover, as highlighted in [47, its dual cone $\Sigma^{*}$ coincides with the Reeb cone (6):

$$
\begin{aligned}
\Sigma^{*} & :=\{K \in \mathfrak{t} \mid \forall x \in \Sigma,\langle x, K\rangle>0\}=\left\{K \in \mathfrak{t} \mid\left\langle\mu_{\eta}, K\right\rangle>0 \text { on } N\right\} \\
& =\{K \in \mathfrak{t} \mid \eta(K)>0 \text { on } N\}=\mathfrak{t}_{+}^{X} .
\end{aligned}
$$

The image of $\mu_{\eta}$ itself is the transversal slice

$$
P_{X}:=\mu_{\eta}(N)=\{x \in \Sigma \mid\langle x, X\rangle=1\}
$$

of the momentum cone; it is thus a compact convex polytope which, following 42, we call the transversal polytope of $\left(N, X, J^{X}, \mathbb{T}\right)$-in [46], it is instead called the characteristic polytope. We thus obtain the following immediate consequences of Lemma 1.13, Definition 1.14 and the duality (7).

Lemma 1.15. Let $\left(N, X, J^{X}, \mathbb{T}\right)$ be a compact of Sasaki type. Then all $\eta \in \mathcal{S}\left(X, J^{X}\right)^{\mathbb{T}}$ have the same momentum cone $\Sigma$ and the same transversal polytope $P_{X}$. Furthermore, the duality (7) identifies $\mathfrak{t}$ with the space of affine-linear functions $A f f\left(P_{X}, \mathbb{R}\right)$ on the transversal polytope $P_{X}$, with $\mathfrak{t}_{+}^{X}$ being the subset of strictly positive such functions.

Remarks 1.16. The fact that $P_{X} \subseteq \mathfrak{t}^{*}$ is independent of the choice of $\eta \in \mathcal{S}\left(X, J^{X}\right)^{\mathbb{T}}$ may also be deduced from the $\mathbb{T}$-equivariant Gray-Moser isotopy argument as in Remark 1.8 .

When $X$ is quasiregular, $P_{X}$ is the (natural) momentum polytope of the Sasaki-Reeb quotient $(M, J, \omega)$ with respect to the induced action of $\mathbb{T} / \mathbb{S}_{X}^{1}$ where $\mathbb{S}_{X}^{1} \leqslant \mathbb{T}$ denotes the $\mathbb{S}^{1}$-action generated by $X$.

More generally, the above discussion may be reinterpreted in the transversal Kähler geometry of $\left(N, X, J^{X}, \mathbb{T}\right)$, where we have in particular the following observation.

Lemma 1.17. Suppose that $N$ is compact, and that $\eta_{0}, \eta \in \mathcal{S}\left(X, J^{X}\right)^{\mathbb{T}}$ are related by (21); then the induced transversal Kähler forms are related by $\omega=\omega_{0}+d_{X} d_{X}^{c} \varphi$, i.e., they belong to the same transversal Kähler class. Moreover any $K \in \mathfrak{t}$ induces a transversal 
Killing field, denoted $\check{K}$, for both $\omega_{0}$ and $\omega$, whose respective transversal Killing potentials $f_{0}:=\eta_{0}(K)$ and $f:=\eta(K)$ are related by

$$
f=f_{0}+d^{c} \varphi(\check{K}) .
$$

Proof. The first part is immediate from (2), and this also implies $f=f_{0}+d^{c} \varphi(K)+\alpha(K)$. Since $\alpha$ is closed with $\mathcal{L}_{K} \alpha=0$, the term $\alpha(K)$ is a constant. Using that $\alpha$ is basic with respect to $X$, the argument of Lemma 1.13 shows that $f_{0}+d^{c} \varphi(K)$ and $f$ agree at a global minimum of $f$, so $\alpha(K)=0$ and (9) holds.

\section{SASAKI STRUCTURES VIA COMPleX CONES}

There is a well-known link between Sasaki structures and conical Kähler metrics, which is used in many places, see e.g. [11, 13, 29, 47].

Let $(N, \mathcal{D}, J, X)$ be a Sasaki manifold and $\mathbb{R}^{+}$the multiplicative group of positive real numbers; then $V=\mathbb{R}^{+} \times N$ has a Kähler structure $(\hat{J}, \hat{\omega}, \hat{g})$ defined as follows. Let $r: V \rightarrow \mathbb{R}^{+}$and $\pi: V \rightarrow N$ denote the projections and let $Z \in C_{V}^{\infty}(T V)$ be the generator of the natural $\mathbb{R}^{+}$action on $V$; then we have direct sum decompositions

$$
T V \cong \mathbb{R} \cdot Z \oplus \pi^{*} T N=\mathbb{R} \cdot Z \oplus \pi^{*}(\mathbb{R} \cdot X) \oplus \pi^{*} \mathcal{D}
$$

and we let $\hat{J}$ be the unique almost complex structure on $V$ which restricts to $\pi^{*} J$ on $\pi^{*} \mathcal{D}$ and sends $Z$ to $\pi^{*} X$. Since $J$ induces a transversal holomorphic structure with respect to $X$ on $N$ and $d \eta_{\mathcal{D}}^{X} \in \Omega_{X}^{(1,1)}(N), \hat{J}$ is integrable: indeed, using $d r(Z)=r$, we have $d^{c} r=r \pi^{*} \eta_{\mathcal{D}}^{X}$ and so $d r+i J r \pi^{*} \eta_{\mathcal{D}}^{X}$ is a $(1,0)$-form on $V$, as are pullbacks by $\pi$ of basic (1,0)-forms on $N$, and together these generate a differential ideal. We now set

$$
\begin{aligned}
\hat{\omega} & :=\frac{1}{2} d\left(r^{2} \pi^{*} \eta_{\mathcal{D}}^{X}\right)=r d r \wedge \pi^{*} \eta_{\mathcal{D}}^{X}+\frac{1}{2} r^{2} \pi^{*} d \eta_{\mathcal{D}}^{X}, \\
\hat{g} & :=-\hat{\omega} \hat{J}=d r^{2}+r^{2} \pi^{*} g_{N}, \quad \text { where } \quad g_{N}:=\left(\eta_{\mathcal{D}}^{X}\right)^{2}+\frac{1}{2} d \eta_{\mathcal{D}}^{X}(\cdot, J \cdot),
\end{aligned}
$$

and these evidently define a Kähler metric on $V$ compatible with $\hat{J}$.

Remarks 2.1. $(V, Z, \hat{J}, \hat{\omega}, \hat{g})$ is sometimes called the Kähler cone of $(N, \mathcal{D}, J, X)$ and $g_{N}$ its Sasaki metric, due to the following more general considerations.

(i) Let $V$ be any smooth manifold equipped with a vector field $Z$; then a riemannian metric $\hat{g}$ or symplectic form $\hat{\omega}$ is conical with respect to $Z$ if $\mathcal{L}_{Z} \hat{g}=2 \hat{g}$ or $\mathcal{L}_{Z} \hat{\omega}=2 \hat{\omega}$ respectively. If in addition $Z$ generates a free proper action of $\mathbb{R}^{+}$(so that $V / \mathbb{R}^{+}$is a manifold) then $(V, Z, \hat{g})$ or $(V, Z, \hat{\omega})$ is called a riemannian or symplectic cone respectively.

(ii) For any riemannian manifold $\left(N, g_{N}\right),\left(\mathbb{R}^{+} \times N, Z, d r^{2}+r^{2} \pi^{*} g_{N}\right)$ is a riemannian cone, where $Z$ generates the $\mathbb{R}^{+}$action and $r, \pi$ are the projections (as above). Conversely, if $(V, \tilde{Z}, \hat{g})$ is a riemannian cone and $\tilde{r}^{2}:=\hat{g}(\tilde{Z}, \tilde{Z})$, there is a unique metric $g_{N}$ on $N=V / \mathbb{R}^{+}$ such that the quotient $\tilde{\pi}$ is a riemannian submersion from $\left(V, \tilde{r}^{-2} \hat{g}\right)$ to $\left(N, g_{N}\right)$; then $(\tilde{r}, \tilde{\pi})$ defines an isomorphism of riemannian cones (an $\mathbb{R}^{+}$-equivariant isometry) from $(V, \tilde{Z}, \hat{g})$ to $\left(\mathbb{R}^{+} \times N, Z, d r^{2}+r^{2} \pi^{*} g_{N}\right)$. In particular, the riemannian cone of a Sasaki metric is Kähler and this is often used as a definition of Sasaki manifolds - see e.g. [47].

(iii) Any co-oriented contact manifold $(N, \mathcal{D})$ has a symplectization $\left(\mathcal{D}_{+}^{0}, \Omega\right)$, where $\mathcal{D}_{+}^{0} \subseteq$ $T^{*} N$ is the component of the annihilator of $\mathcal{D} \subseteq T N$ distinguished by the co-orientation, and $\Omega$ is the pullback to $\mathcal{D}_{+}^{0}$ of the canonical Liouville symplectic form on $T^{*} N$. Then (see e.g. [44]) $\left(\mathcal{D}_{+}^{0}, Z, \Omega\right)$ is a symplectic cone, where $Z$ is the generator of $\mathbb{R}^{+}$action $(\lambda, p) \mapsto \lambda^{2} p$, for $\lambda \in \mathbb{R}^{+}$and $p \in \mathcal{D}_{+}^{0}$ (thus $Z$ is twice the generator of the natural action by scalar multiplication). Conversely any symplectic cone $(V, \tilde{Z}, \hat{\omega})$ arises in this way up to isomorphism $\left(\mathbb{R}^{+}\right.$-equivariant symplectomorphism). 
In this section, which is a synthesis of approaches in He-Sun [36], Collins-Székelyhidi [18] and Boyer-van Coevering [14], we study the cone $V$ from a complex viewpoint. In contrast to symplectic and riemannian cones, $(V, Z, \hat{J})$ cannot be constructed using only the transversal holomorphic structure of $N$, but this proves to be fortuitous for our purposes, as the additional data needed is a marking in the sense of Definition 1.11.

2.1. Complex cones and cone potentials. In order to characterize complex cones $(V, Z, \hat{J})$ and their compatible Kähler cone metrics $(\hat{g}, \hat{\omega})$, a key fact is that for any such cone metric, the positive function $r$ defined by

$$
r^{2}:=\hat{g}(Z, Z)=\hat{\omega}(Z, \hat{J} Z)
$$

satisfies

$$
\mathcal{L}_{X} r=0, \quad \mathcal{L}_{Z} r=r, \quad \hat{\omega}=\frac{1}{4} d d^{c}\left(r^{2}\right),
$$

where $X=\hat{J} Z$. Thus the function $r$ suffices to define $\hat{\omega}$, and hence $\hat{g}=-\hat{\omega} \hat{J}$, which will be a compatible Kähler metric on $(V, \hat{J})$ provided $d d^{c}\left(r^{2}\right)>0$, i.e., $r^{2}$ is (strictly) plurisubharmonic. Henceforth we drop the hat on $J$ and describe compatible cone metrics purely in terms of such functions $r$. We also take the vector field $X$ (not $Z$ ) as primitive.

Definition 2.2. On a complex manifold $(V, J)$ with a holomorphic vector field $X$, we let

$$
\mathcal{R}_{X}(V, J):=\left\{r \in C_{V}^{\infty}\left(\mathbb{R}^{+}\right): \mathcal{L}_{X} r=0, \mathcal{L}_{-J X} r=r, d d^{c} r>0\right\}
$$

be the space of (radial) cone potentials ( $X$-invariant plurisubharmonic functions of homogeneity 1 with respect to $-J X)$. If $(V, J, X)$ admits a surjective cone potential $r: V \rightarrow \mathbb{R}^{+}$, then $X$ is called a polarization of $(V, J)$ and $(V, J, X)$ is called a polarized complex cone.

Example 2.3. Let $\left(N, \mathcal{D}_{0}, J_{N}, X\right)$ be a regular Sasaki manifold as in Example 1.3, and let $\left(M, J_{M}, \omega_{0}\right)$ be its Sasaki-Reeb quotient, a compact Kähler manifold. Associated to the principal $\mathbb{S}^{1}$-bundle $\pi: N \rightarrow M$ is a holomorphic line bundle $\pi: L \rightarrow M$, endowed with a hermitian product $h$, such that Chern curvature 2-form of $(L, h)$ is $\omega_{0}$. It this situation, $N$ can be identified with the subset of unit vectors in the dual hermitian line bundle $\left(L^{*}, h^{-1}\right)$, with its induced hypersurface CR structure and Sasaki-Reeb vector field $X=J Z$, where $Z$ generates the natural $\mathbb{R}^{+}$-action on the bundle $L^{*}$. Furthermore, the conical Kähler metric associated to the Sasaki manifold $N$ is isomorphic ( $\mathbb{R}^{+}$-equivariantly isometric) to $\left(\left(L^{*}\right)^{\times}, Z\right)$ with the metric $\left(\hat{\omega}=\frac{1}{4} d d^{c}\left(r_{h}^{2}\right), \hat{g}=-\hat{\omega} J\right)$, where $V=\left(L^{*}\right)^{\times}$is the complex manifold obtained from the total space of $L^{*}$ by removing the zero section, and $r_{h}$ is the norm function on $L^{*}$ defined by the hermitian metric $h^{-1}$. Thus, in this case, the polarized complex cone is $\left(\left(L^{*}\right)^{\times}, X\right)$ where $X$ is the generator of the fibrewise $\mathbb{S}^{1}$-action on $L^{*}$. Furthermore, we can identify the space $\mathcal{R}_{X}(V, J)$ with the space of hermitian metrics on the ample line bundle $L$ whose curvature form $\omega$ is positive definite, whereas the space $\hat{\Xi}\left(X, J^{X}, \eta_{0}^{X}\right)$, where $\eta_{0}^{X}$ is the contact form of $\left(\mathcal{D}_{0}, X\right)$, is isomorphic to the space $\left\{f \in C_{M}^{\infty}(\mathbb{R}) \mid \omega=\omega_{0}+d d^{c} f>0\right\}$ of relative Kähler potentials on $(M, J, \omega)$.

We want to show more generally that $\mathcal{R}_{X}(V, J)$ is a basepoint free version of the space $\hat{\Xi}\left(X, J^{X}, \eta_{0}^{X}\right)$ of Sasaki potentials (4). We begin with some straightforward observations.

Lemma 2.4. Let $(V, J, X)$ be a polarized complex cone. Then:

(i) $Z:=-J X$ generates a free proper action of $\mathbb{R}^{+}$and we let $N^{X}=V / \exp (-J X)$ be the quotient manifold with quotient map $\pi_{X}: V \rightarrow N^{X}$;

(ii) $X$ descends to a vector field on $N^{X}$, which we also denote by $X$, and $J$ to a transversal holomorphic structure $J^{X}$ on $\mathcal{D}_{X}=T N^{X} / \mathbb{R} \cdot X$;

(iii) for all $r \in \mathcal{R}_{X}(V, J)$ and $c \in \mathbb{R}^{+}, N^{X}$ is diffeomorphic to $r^{-1}(c)$, so any such $r: V \rightarrow$ $\mathbb{R}^{+}$is surjective and $\left(r, \pi_{X}\right): V \rightarrow \mathbb{R}^{+} \times N^{X}$ is a diffeomorphism; 
(iv) $N^{X}$ is compact if and only if some, hence any, cone potential $r: V \rightarrow \mathbb{R}^{+}$is proper. Conversely if $(V, J)$ is a complex manifold with a holomorphic vector field $X$ such that $-J X$ generates a proper action of $\mathbb{R}^{+}$, then any cone potential is surjective, and so $(V, J, X)$ is a polarized complex cone if and only if $\mathcal{R}_{X}(V, J)$ is nonempty.

Proof. (i) and (iii) follow because $-J X$ is transverse to the level surfaces of any cone potential $r$, (ii) because $X, Z$ are holomorphic with $[X, Z]=0$, (iv) by definition, and the converse by the equivariance of cone potentials with respect to the $\mathbb{R}^{+}$action.

For any $r \in C_{V}^{\infty}\left(\mathbb{R}^{+}\right)$, we set $N_{r}:=r^{-1}(1)$ and $\tilde{\eta}_{r}:=d^{c} r / r$.

Lemma 2.5. Let $(V, J)$ be a complex manifold with a holomorphic vector field $X$ and a function $r \in C_{V}^{\infty}\left(\mathbb{R}^{+}\right)$such that $\mathcal{L}_{X} r=0$ and $\mathcal{L}_{-J X} r=r$. Then $\tilde{\eta}_{r}$ is the pullback $\pi_{X}^{*} \eta_{r}$ of a 1-form $\eta_{r}$ on $N^{X}$ with $\eta_{r}(X)=1$ and $\mathcal{L}_{X} \eta_{r}=0$, hence also $\iota_{X} d \eta_{r}=0$. Further, for any $\lambda \in \mathbb{R}^{+}$the following are equivalent:

(i) $r$ is plurisubharmonic, i.e., is in $\mathcal{R}_{X}(V, J)$;

(ii) $r^{\lambda}$ is plurisubharmonic, hence is in $\mathcal{R}_{X / \lambda}(V, J)$;

(iii) $\eta_{r} \in \mathcal{S}\left(X, J^{X}\right)$.

Thus $r \mapsto r^{\lambda}$ is a bijection from $\mathcal{R}_{X}(V, J)$ to $\mathcal{R}_{X / \lambda}(V, J)$, and if $r \in \mathcal{R}_{X}(V, J),\left.\pi_{X}\right|_{N_{r}}$ is a CR isomorphism from $N_{r}$ (with the hypersurface $C R$ structure) to $N^{X}$ (with the $C R$ structure induced by $J^{X}$ and $\mathcal{D}_{r}=\operatorname{ker} \eta_{r}$, i.e., by $\eta_{r} \in \mathcal{S}\left(X, J^{X}\right)$ ).

Proof. Set $\rho=\log r$. For the first part, we compute $\mathcal{L}_{X} \rho=0$ and $\mathcal{L}_{-J X} \rho=1$, from which it follows that $\tilde{\eta}_{r}$ is basic with respect to the holomorphic vector field $Z=-J X$. Hence it is a pullback as stated, and the properties of $\eta_{r}$ follow straightforwardly. For the equivalence, observe that the function $r^{\lambda}=e^{\lambda \rho}$ satisfies $\mathcal{L}_{X / \lambda}\left(r^{\lambda}\right)=0, \mathcal{L}_{-J X / \lambda}\left(r^{\lambda}\right)=r^{\lambda}$, and

$$
d d^{c}\left(r^{\lambda}\right)=\lambda d\left(r^{\lambda} \tilde{\eta}_{r}\right)=\lambda r^{\lambda}\left(\lambda(d r / r) \wedge \pi_{X}^{*} \eta_{r}+\pi_{X}^{*} d \eta_{r}\right)
$$

This is positive if and only if $d \eta_{r}$ is positive on ker $\eta_{r}$. Thus (ii) is equivalent to (iii), hence also (i) by taking $\lambda=1$.

The bijection follows immediately, and the last part follows because $T N_{r}=\left.\operatorname{ker} d r\right|_{N_{r}}=$ ker $\left.d \rho\right|_{N_{r}}$, so the hypersurface CR structure has contact distribution ker $\left.\tilde{\eta}_{r}\right|_{N_{r}}$ with the complex structure induced by $J$, hence is $\pi_{X}$-related to $\mathcal{D}_{r}$ with the complex structure induced by $J^{X}$.

Corollary 2.6. If $(V, J, X)$ is a polarized complex cone, then the induced transversal holomorphic structure $\left(X, J^{X}\right)$ on $N^{X}$ has Sasaki type.

Remark 2.7. For any cone potential $r \in \mathcal{R}_{X}(V, J)$, and any $a, \lambda \in \mathbb{R}^{+}, d d^{c}\left(a r^{\lambda}\right)$ is thus a Kähler metric on $(V, J)$. To obtain a conical Kähler metric of homogeneity 2 with respect to $Z=-J X$, we take $\lambda=2$ and (conventionally) $c=1 / 4$ so that the Kähler form is

$$
\omega_{r}:=\frac{1}{4} d d^{c}\left(r^{2}\right)=\frac{1}{2} d\left(r^{2} \tilde{\eta}_{r}\right)=r d r \wedge \tilde{\eta}_{r}+\frac{1}{2} r^{2} d \tilde{\eta}_{r},
$$

and $\frac{1}{2} r^{2}=\frac{1}{2} \omega_{r}(X, J X)$ is a Killing potential for $X$. However, we generally find it simpler to work directly with the cone potential $r$ than a conventional choice of conical metric.

Lemma 2.8. Let $(V, J, X)$ be a polarized complex cone, $r_{0} \in \mathcal{R}_{X}(V, J), r \in C_{V}^{\infty}\left(\mathbb{R}^{+}\right)$, and $\eta_{0}:=\eta_{r_{0}} \in \mathcal{S}\left(X, J^{X}\right)$. Then $r \in \mathcal{R}_{X}(V, J)$ if and only if there exists a Sasaki potential $\varphi \in \hat{\Xi}\left(X, J^{X}, \eta_{0}\right)$ such that $r=\exp \left(\pi_{X}^{*} \varphi\right) r_{0}$, and then $\eta_{r}=\eta_{0}+d^{c} \varphi$.

Proof. We may write $r=\exp (\tilde{\varphi}) r_{0}$, for some $\tilde{\varphi} \in C_{V}^{\infty}(\mathbb{R}) ;$ then $\mathcal{L}_{-J X} r=r$ and $\mathcal{L}_{X} r=0$ hold if and only if $\tilde{\varphi}=\pi_{X}^{*} \varphi$ with $\mathcal{L}_{X} \varphi=0$. We then have $\eta_{r}=\eta_{0}+d^{c} \varphi$ and the result follows by Lemma 2.5 . 
Note that $\varphi$ in Lemma 2.8 is determined by $\pi_{X}^{*} \varphi=\log r-\log r_{0}$. Thus the above observations yield the following crucial descriptions of $\mathcal{R}_{X}(V, J)$.

Proposition 2.9. Let $(V, J, X)$ be a polarized complex cone. Then:

(i) the image of $r \mapsto \eta_{r}: \mathcal{R}_{X}(V, J) \rightarrow \mathcal{S}\left(X, J^{X}\right)$ is a marking $\Xi$ of $\left(N, X, J^{X}\right)$ in the sense of Definition 1.11, and $\eta_{r}=\eta_{r_{0}}$ if and only if $r=e^{a} r_{0}$ with $a \in \mathbb{R}$ constant;

(ii) for any $r_{0} \in \mathcal{R}_{X}(V, J)$, we have $\Xi=\Xi\left(X, J^{X}, \eta_{0}\right)$ with $\eta_{0}=\eta_{r_{0}}$, and the map

$$
r \in \mathcal{R}_{X}(V, J) \mapsto \varphi \in C_{N}^{\infty}(\mathbb{R}) \quad \text { with } \quad \pi_{X}^{*} \varphi=\log r-\log r_{0}
$$

is a bijection from $\mathcal{R}_{X}(V, J)$ to the space $\hat{\Xi}\left(X, J^{X}, \eta_{0}\right)$ of Sasaki potentials (4) on $N^{X}$;

(iii) $r \mapsto N_{r}$ is a bijection from $\mathcal{R}_{X}(V, J)$ to the set of strictly pseudoconvex (images of) $X$-invariant sections of $\pi_{X}$.

Proof. (i)-(ii) are immediate from Lemma 2.8 and the connectedness of $V$.

(iii) By Lemma 2.5, for any $r \in \mathcal{R}_{X}(V, J), N_{r}$ is strictly pseudoconvex and is the image of an $X$-invariant section of $\pi_{X}$. Conversely given such a section, the condition that $r=1$ on the image and that $\mathcal{L}_{-J X} r=r$ determine that $r=e^{\rho}$ where $\rho(y)$ is the time for the flow of $J X$ starting at $y \in V$ to meet the section. Since the section is $X$-invariant and $X$ is holomorphic, $r$ so defined is a smooth function on $V$ with $\mathcal{L}_{X} r=0$ and $\mathcal{L}_{-J X} r=r$; now $d d^{c} r$ is positive at $r=1$ by pseudoconvexity of the image of the section, hence it is positive on $V$, since it is homogeneous of degree 1 with respect to $Z=-J X$.

Part (i) of this result shows that there is a canonical bijection up to isomorphism 1 between polarized complex cones $(V, J, X)$ and marked transversal holomorphic manifolds $\left(N, X, J^{X}, \Xi\right)$, where an isomorphism of polarized complex cones is an $\mathbb{R}^{+}$-equivariant biholomorphism of the corresponding manifolds, and an isomorphism of marked transversal holomorphic manifolds is a diffeomorphism of corresponding manifolds intertwining their transversal holomorphic structures and markings.

Part (ii) shows that $\mathcal{R}_{X}(V, J)$ is an (infinite dimensional) affine manifold with a global affine chart $\hat{\Xi}\left(X, J^{X}, \eta_{0}\right)$-indeed the space $\left\{\rho=\log r \mid r \in \mathcal{R}_{X}(V, J)\right\}$ of logarithmic cone potentials is an affine space modelled on $\hat{\Xi}\left(X, J^{X}, \eta_{0}\right)$.

2.2. Reeb cones revisited. Let $(V, J, X)$ be a polarized complex cone with corresponding marked transversal holomorphic manifold $\left(N^{X}, X, J^{X}, \Xi\right)$, assumed compact. In particular $\left(X, J^{X}\right)$ has Sasaki type (Corollary 2.6) and so, as in \$1.3, we may fix a compact torus $\mathbb{T} \leqslant$ $\operatorname{Aut}\left(N, X, J^{X}\right)$, with $X \in \mathfrak{t}=\operatorname{Lie}(\mathbb{T})$, which preserves some compatible Sasaki structure $\eta_{0} \in \Xi$. Thus $X, J^{X}$, and $\Xi \cong \Xi\left(X, J^{X}, \eta_{0}\right)$ are $\mathbb{T}$-invariant, hence there is an induced holomorphic action of $\mathbb{T}$ on $(V, J, X)$. We denote by $\Xi^{\mathbb{T}}$ the space of $\mathbb{T}$-invariant elements of $\Xi$ (thus $\Xi^{\mathbb{T}} \cong \Xi\left(X, J^{X}, \eta_{0}\right)^{\mathbb{T}}$ for any $\left.\eta_{0} \in \Xi^{\mathbb{T}}\right)$ and by $\mathcal{R}_{X}(V, J)^{\mathbb{T}}$ the space of $\mathbb{T}$-invariant cone potentials in $\mathcal{R}_{X}(V, J)$. The considerations of the previous subsection apply mutatis mutandis in the $\mathbb{T}$-invariant context to show that

(i) $r \mapsto \eta_{r}$ is a surjection with 1-dimensional fibres from $\mathcal{R}_{X}(V, J)^{\mathbb{T}}$ to $\Xi^{\mathbb{T}} \subseteq \mathcal{S}\left(X, J^{X}\right)^{\mathbb{T}}$;

(ii) for any $\eta_{0} \in \Xi^{\mathbb{T}}, \mathcal{R}_{X}(V, J)^{\mathbb{T}}$ is isomorphic to the space $\hat{\Xi}\left(X, J^{X}, \eta_{0}\right)^{\mathbb{T}}$ of $\mathbb{T}$-invariant Sasaki potentials;

(iii) $r \mapsto N_{r}$ identifies $\mathcal{R}_{X}(V, J)^{\mathbb{T}}$ with the space of $\mathbb{T}$-invariant sections of $\pi_{X}: V \rightarrow N^{X}$.

Let $\mathfrak{t}_{+}^{X} \subseteq \mathfrak{t}$ the Reeb cone of $\mathbb{T}$ (Definition 1.14). An important observation in [14, 18] is that $\mathfrak{t}_{+}^{X}$ can be defined intrinsically on $(V, J, \mathbb{T})$, and indeed can be identified with

$$
\mathfrak{t}_{+}:=\{K \in \mathfrak{t} \mid K \text { is a polarization of }(V, J)\} \text {. }
$$

\footnotetext{
${ }^{1}$ It is only an equivalence of categories if one works with the quotient groupoid of $\mathbb{R}^{+}$-equivariant isomorphisms of polarized complex cones by the action of $\mathbb{R}^{+}$.
} 
To establish this, it is useful to reformulate the definition of $\mathfrak{t}_{+}^{X}$ on $V$ (see e.g. [36]).

Lemma 2.10. For any $r \in \mathcal{R}_{X}(V, J)^{\mathbb{T}}$,

$$
\mathfrak{t}_{+}^{X}=\left\{K \in \mathfrak{t} \mid \mathcal{L}_{-J K} r>0\right\}=\left\{K \in \mathfrak{t} \mid J K \text { is transverse to } N_{r}:=r^{-1}(1)\right\} .
$$

Consequently, $(V, J)$ has a proper holomorphic action of a complex torus $\mathbb{T}_{\mathbb{C}}$ whose real part coincides with $\mathbb{T}$. Furthermore $\mathfrak{t}_{+}^{X} \subseteq \mathfrak{t}_{+}$, and $K \in \mathfrak{t}_{+}^{X}$ if and only if $X \in \mathfrak{t}_{+}^{K}$.

Proof. Since $\mathcal{L}_{-J X} r=\left(d^{c} r\right)(X)=r\left(\pi_{X}^{*} \eta_{r}\right)(X)$, the first equality in 14 is immediate, and the second follows because $\pi_{X}^{*} \eta_{r}$ is a pullback and $N_{r}$ is a section of $\pi_{X}$.

It follows that for any $K \in \mathfrak{t}_{+}, J K$ is a complete vector field on $(V, J)$. As $\mathfrak{t}_{+}$is open in $\mathfrak{t}$, this gives the second claim. Now for $K \in \mathfrak{t}_{+}^{X}, N_{r}$ is a $\mathbb{T}$-invariant section of $\pi_{K}$ which yields a surjective cone potential $\tilde{r} \in \mathcal{R}_{K}(V, J)$ by Proposition 2.9)(iii) and Lemma 2.4, so $K \in \mathfrak{t}_{+}$. As $N_{\tilde{r}}:=\tilde{r}^{-1}(1)=N_{r}$ is a section of $\pi_{X}, X \in \mathfrak{t}_{+}^{K}$; the converse holds symmetrically.

Remark 2.11. It follows straightforwardly (cf. [36, Lemma 2.3]) that $\mathfrak{t}_{+}^{X}$ is a connected component of $\mathfrak{t}_{+}$. Indeed, for $K \in \mathfrak{t}_{+}$, the condition to be in $\mathfrak{t}_{+}^{X}$ is open because $N_{r}$ is compact. Suppose now that $K$ is a limit point of $\mathfrak{t}_{+}^{X}$ which is not in $\mathfrak{t}_{+}^{X}$. Then $\pi_{X}^{*} \eta_{r}(K) \geqslant 0$ on $N_{r}$, but $\exists p \in N_{r}$ with $\eta_{r}(K)\left(\pi_{X}(p)\right)=0$. Since this is a minimum $d\left(\eta_{r}(K)\right)_{\pi_{X}(p)}=0$ and hence $d \eta_{r}(K, \cdot)_{\pi_{X}(p)}=0$ as $K \in \mathfrak{t}$ preserves $\eta_{r}$. If $K \in \mathfrak{t}_{+}$, with surjective cone potential $\tilde{r} \in \mathcal{R}_{K}(V, J)$, then

$$
d \tilde{r} \wedge \pi_{K}^{*}\left(\eta_{\tilde{r}} \wedge d \eta_{\tilde{r}}^{\wedge m}\right)=f d r \wedge \pi_{X}^{*}\left(\eta_{r} \wedge d \eta_{r}^{\wedge m}\right)
$$

for some function $f$ on $V$. Contracting with $K$ and evaluating at $p$ then yields $\left(d \eta_{\tilde{r}}^{\wedge m}\right)_{\pi_{K}(p)}=$ 0 , which contradicts Lemma 2.5. Thus $\mathfrak{t}_{+}^{X}$ is both open and closed in $\mathfrak{t}_{+}$.

To prove $\mathfrak{t}_{+} \subseteq \mathfrak{t}_{+}^{X}$, we proceed indirectly, following [14, 18], by introducing another equivalent definition of the Reeb cone. To explain this, we first observe if $\Lambda \leqslant \mathfrak{t}$ is the lattice of circle subgroups of $\mathbb{T}$, then $\left(\mathbb{R}^{+} \cdot \Lambda\right) \cap \mathfrak{t}_{+}$is dense in $\mathfrak{t}_{+}$, and we may thus assume $X$ is quasiregular ( $\mathfrak{t}_{+}^{X}$ varies continuously with $X$ by (14) - indeed it is locally constant in $X$ by Remark 2.11). Then, similarly to the regular case (Example 2.3), $V$ can be identified with the space of nonzero vectors in the dual of an orbi-ample orbiline bundle $L$ over the compact Kähler orbifold $\left(M, J_{M}, \omega_{0}\right)$ which is the Sasaki-Reeb quotient of $N$ by the circle action generated by $X$. Thus $V$ has a natural one point compactification $\widehat{V}=V \cup\{0\}$ which the singular space obtained by blowing down the zero section in the total space of $L^{*}$. Since cone potentials correspond to norms of hermitian metrics on $L$, for any $r \in \mathcal{R}_{X}(V, J)$, the apex 0 of the cone $\widehat{V}$ is characterized as the limit of points $p \in V$ with $r(p) \rightarrow 0$. Let

$$
\mathscr{H} \cong \bigoplus_{k \in \mathbb{N}} H^{0}\left(M, L^{k}\right)
$$

be the space of continuous complex-valued functions of $\widehat{V}$ which are holomorphic on $V \cong$ $\left(L^{*}\right)^{\times}$and polynomial on each fibre of $\left(L^{*}\right)^{\times}$over $M$. Here $H^{0}\left(M, L^{k}\right)$ is the space of holomorphic sections $s$ of $L^{k}$, which define fibrewise polynomial functions $f_{s}$ on $L^{*}$ by $f_{s}(p)=\left\langle s, p^{k}\right\rangle$. Now $\mathbb{T}_{\mathbb{C}}$ acts on $\mathscr{H}$ and for $\alpha \in \mathfrak{t}^{*}$ we let

$$
\mathscr{H}_{\alpha}:=\left\{f \in \mathscr{H} \mid \forall K \in \mathfrak{t}, \mathcal{L}_{-J K} f=\alpha(K) f\right\}
$$

be the $\alpha$-weight space and

$$
\Gamma:=\left\{\alpha \in \mathfrak{t}^{*} \mid \mathscr{H}_{\alpha} \neq 0\right\}
$$

be the set of (integral) weights of the action. Thus there is a weight space decomposition

$$
\mathscr{H} \cong \bigoplus_{\alpha \in \Gamma} \mathscr{H}_{\alpha}
$$


where the degree $k$ component of $(15)$ is the direct sum of the weight spaces $\mathscr{H}_{\alpha}$ with $\alpha(X)=k$. The key fact we need is that (by ampleness of $L) \mathscr{H}$ separates points of $\widehat{V}$ : in particular for any $p \in \widehat{V}$ there exists $f \in \mathscr{H}$ with $f(0)=0$ and $f(p) \neq 0$.

Remark 2.12. In fact orbifold versions of the Kodaira embedding theorem (see [50]) embed $\widehat{V}$ as an affine variety in $\mathbb{C}^{N}$ which is a cone with the singular apex at the origin. The functions in $\mathscr{H}$ are then the regular functions on $\widehat{V}$, which separate points by definition.

The incisive idea in [18] is to define the Reeb cone in $\mathfrak{t}$ as dual to the cone generated by $\Gamma$ in $\mathfrak{t}^{*}$, i.e., to set

$$
\mathfrak{t}_{+}^{\Gamma}=\{K \in \mathfrak{t} \mid \forall \alpha \in \Gamma \backslash\{0\}, \alpha(K)>0\} .
$$

Now [18, Prop. 2.1] asserts that $\mathfrak{t}_{+}^{\Gamma}=\mathfrak{t}_{+}^{X}$, while [14, Prop. 2] asserts that $\mathfrak{t}_{+}^{\Gamma}=\mathfrak{t}_{+}$, which are the results we need. However, [14] refers to [18] for the inclusion $\mathfrak{t}_{+} \subseteq \mathfrak{t}_{+}^{\Gamma}$, while we have been unable to verify the details of the argument in [18] that $\mathfrak{t}_{+}^{\Gamma} \subseteq \mathfrak{t}_{+}^{X}$. Since these claims are crucial for our work, for the convenience of the reader we present proofs here.

Lemma 2.13. If $X \in \mathfrak{t}_{+}$is quasiregular then $\mathfrak{t}_{+} \subseteq \mathfrak{t}_{+}^{\Gamma}$ and $\mathfrak{t}_{+}^{\Gamma} \subseteq \mathfrak{t}_{+}^{X}$.

Proof. For $\mathfrak{t}_{+} \subseteq \mathfrak{t}_{+}^{\Gamma}$, we follow the main ideas in the proof of [18, Prop. 2.1]. Let $K \in \mathfrak{t}_{+}$ and $r \in \mathcal{R}_{K}(V, J)$. As noted already, $\mathcal{L}_{-J K} r=r$ implies $r$ is equivariant with respect to the $\mathbb{R}^{+}$action generated by $-J K$. Thus under the flow $\Phi_{t}^{-J K}$ of $-J K$, for any $p \in V$,

$$
r\left(\Phi_{t}^{-J K}(p)\right) \rightarrow 0 \text { as } t \rightarrow 0
$$

and so $\Phi_{t}^{-J K}(p) \rightarrow 0$, the apex of $\widehat{V}$. Now let $\alpha \in \Gamma \backslash\{0\}$ and $f \in \mathscr{H}_{\alpha} \backslash\{0\}$. Then $\mathcal{L}_{-J K} f=\alpha(K) f, f(0)=0$, and there exists $p \in V$ with $f(p) \neq 0$. Since

$$
\frac{d}{d t} f\left(\Phi_{t}^{-J K}(p)\right)=\alpha(K) f\left(\Phi_{t}^{-J K}(p)\right)
$$

and $f\left(\Phi_{t}^{-J K}(p)\right) \rightarrow f(0)=0$ as $t \rightarrow 0$, we must have $\alpha(K)>0$. Thus $K \in \mathfrak{t}_{+}^{\Gamma}$.

For $\mathfrak{t}_{+}^{\Gamma} \subseteq \mathfrak{t}_{+}^{X}$, we give a novel argument. Let $K \in \mathfrak{t}_{+}^{\Gamma}$ and $r \in \mathcal{R}_{X}(V, J)$. By (7), it suffices to show that $K$ is in the dual of the momentum cone of the contact momentum map of $\eta_{r}$ on $N^{X}$ (Definition 1.14). For this, we first let $p \in N_{r}$ have maximal stabilizer with respect to the $\mathbb{T}$-action, i.e., $\mathfrak{t}=\mathfrak{s t a b}_{\mathbb{T}}(p) \oplus \mathbb{R} \cdot X$. Thus $\left\{Y_{p} \mid Y \in \mathfrak{t}\right\}=\mathbb{R} \cdot X_{p}$ and $K_{p}-\eta_{r}(K)_{\pi_{X}(p)} X_{p} \in \mathfrak{s t a b}_{\mathbb{T}}(p)$. Since $\mathscr{H}$ separates points, there exists $f \in \mathscr{H}_{\alpha}$ with $\alpha \in \Gamma \backslash\{0\}$ and $f(p) \neq 0$. Let $\Phi_{t}^{-J K}$ be the flow of $-J K$ as before and observe that

$$
f\left(\Phi_{t}^{-J K}(p)\right)=f\left(\Phi_{t}^{-\eta_{r}(K)_{\pi_{X}(p)} J X}(p)\right)
$$

so that

$$
\alpha(K) f(p)=\eta_{r}(K)_{\pi_{X}(p)} \alpha(X) f(p) .
$$

Since $\alpha(X)>0$ and $\alpha(K)>0$ by assumption, we have $\eta_{r}(K)_{\pi_{X}(p)}>0$. We conclude that $\langle\xi, K\rangle>0$ at points $\xi=\mu_{\eta_{r}}(p)$ on the edges of the momentum cone. Since the momentum cone is a strictly convex polyhedral cone, the result follows.

Since $\mathfrak{t}_{+}^{X} \subseteq \mathfrak{t}_{+}$by Lemma 2.10, it follows that $\mathfrak{t}_{+}^{X}=\mathfrak{t}_{+}^{\Gamma}=\mathfrak{t}_{+}$. Henceforth we drop superscripts and refer to $\mathfrak{t}_{+}$(with these interchangeable definitions) as the Reeb cone of $(V, J)$. We also note that the momentum cone of any $\eta \in \mathcal{S}\left(X, J^{X}\right)$ coincides with the weight cone generated by $\Gamma$.

Remark 2.14. For any $r \in \mathcal{R}_{X}(V, J)^{\mathbb{T}}, \hat{\omega}:=\frac{1}{4} d d^{c}\left(r^{2}\right)$ is a $\mathbb{T}$-invariant exact symplectic form (see Lemma 2.5), so the $\mathbb{T}$-action on $(V, \hat{\omega})$ is hamiltonian with momentum map $\mu_{r}: V \rightarrow \mathfrak{t}^{*}$ defined by

$$
\left\langle\mu_{r}, K\right\rangle=\frac{1}{4} d^{c}\left(r^{2}\right)(K)=\frac{1}{2} r d^{c} r(Y)
$$


for $K \in \mathfrak{t}$. It is in fact the unique momentum map which is homogeneous of order 2 with respect to the $\mathbb{R}^{+}$-action generated by $-J X$. In our setting, for $p \in N_{r}$,

$$
\mu_{r}(p)=\frac{1}{2} \mu_{\eta_{r}}\left(\pi_{X}(p)\right)
$$

In particular $d^{c} r(K)=r$ so $\frac{1}{2} r^{2}$ is a Killing potential for $K$. Hence the flow of $-J K$ is the gradient flow of the positive function $\frac{1}{2} r^{2}$ with respect to the Kähler metric $\hat{g}=-\hat{\omega} J$.

Observe that $\left\langle\mu_{r}(p), X\right\rangle=1 / 2$ for any $r \in \mathcal{R}_{X}(V, J)^{\mathbb{T}}$ and for any $p \in N_{r}$ thanks to the homogeneity condition (10), so $N_{r}$ has momentum image $(1 / 2) P_{X}$.

2.3. Change of polarization. The $\mathbb{T}$-invariant version of Proposition 2.9(iii) and the final part of Lemma 2.10 have the following crucial corollary, which develops an idea used by He-Sun in the proof of [36, Lemma 2.2].

Corollary 2.15. Let $(V, J, X)$ be the polarized complex cone of a compact $\mathbb{T}$-invariant Sasaki manifold $(N, \mathcal{D}, J, X)$ with $X \in \mathfrak{t}$, and let $K \in \mathfrak{t}_{+}$. Then there is a bijection

$$
\begin{aligned}
\Theta: \mathcal{R}_{X}(V, J)^{\mathbb{T}} & \rightarrow \mathcal{R}_{K}(V, J)^{\mathbb{T}} \\
r & \mapsto \tilde{r}
\end{aligned}
$$

between $\mathbb{T}$-invariant cone potentials with respect to $X$ and such potentials with respect to $K$, characterized uniquely by $\Theta(r)=\tilde{r}$ if and only if $N_{\tilde{r}}:=\tilde{r}^{-1}(1)$ is equal to $N_{r}:=r^{-1}(1)$.

Fixing base points $r_{0} \in \mathcal{R}_{X}(V, J)^{\mathbb{T}}$ and $\tilde{r}_{0}:=\boldsymbol{\Theta}\left(r_{0}\right) \in \mathcal{R}_{K}(V, J)^{\mathbb{T}}$, we also have a bijection

$$
\begin{aligned}
\hat{\Theta}: \hat{\Xi}\left(X, J^{X}, \eta_{r_{0}}\right)^{\mathbb{T}} & \rightarrow \hat{\Xi}\left(K, J^{K}, \eta_{\tilde{r}_{0}}\right)^{\mathbb{T}} \\
\varphi & \mapsto \psi \text { with } \quad \boldsymbol{\Theta}\left(e^{\varphi} r_{0}\right)=e^{\psi} \tilde{r}_{0}
\end{aligned}
$$

such that $\varphi$ and $\hat{\boldsymbol{\Theta}}(\varphi)$ induce Sasaki structures with the same underlying CR structure.

Remark 2.16. From the contact viewpoint (see Remark $\underline{\text { A.2) }}$ ), the images of $\Xi\left(X, J^{X}, \eta_{r_{0}}\right)^{\mathbb{T}}$ and $\Xi\left(K, J^{K}, \eta_{\tilde{r}_{0}}\right)^{\mathbb{T}}$ in $\mathcal{C}_{+}\left(N, \mathcal{D}_{0}\right)^{\mathbb{T}}$ are both formal complexifications of the same orbit of the group $\operatorname{Con}\left(N, \mathcal{D}_{0}\right)^{\mathbb{T}}$ on this space, which does not depend on the choice $X$ or $K$ of Reeb vector field. Hence we expect these images to coincide up to isomorphism. Thus Corollary 2.15 provides an explicit realisation of this correspondence on the level of potentials.

We now compute the derivatives of $\boldsymbol{\Theta}$ and $\hat{\boldsymbol{\Theta}}$. To do this, we note that $T_{r} \mathcal{R}_{X}(V, J)^{\mathbb{T}} \cong$ $C_{N^{X}}^{\infty}(\mathbb{R})^{\mathbb{T}}$, which is isomorphic to $C_{N_{r}}^{\infty}(\mathbb{R})^{\mathbb{T}}$ using pullback by $\Psi_{r}^{X}:=\left.\pi_{X}\right|_{N_{r}}: N_{r} \cong N^{X}$.

Lemma 2.17. Suppose $r_{t}$ is a smooth curve in $\mathcal{R}_{X}(V, J)^{\mathbb{T}}$ with derivative $\dot{r}_{t} \in C_{N^{X}}^{\infty}(\mathbb{R})^{\mathbb{T}}$. Then $\tilde{r}_{t}:=\boldsymbol{\Theta}\left(r_{t}\right)$ is a smooth curve in $\mathcal{R}_{K}(V, J)^{\mathbb{T}}$ with derivative

$$
\dot{\tilde{r}}_{t}=\left(\Psi_{r_{t}}^{X} \circ\left(\Psi_{r_{t}}^{K}\right)^{-1}\right)^{*}\left(\frac{\dot{r}_{t}}{\eta_{r_{t}}(K)}\right) ; \quad \text { in addition } \quad \eta_{\tilde{r}_{t}}=\left(\Psi_{r_{t}}^{X} \circ\left(\Psi_{r_{t}}^{K}\right)^{-1}\right)^{*}\left(\frac{\eta_{r_{t}}}{\eta_{r_{t}}(K)}\right) \text {. }
$$

Proof. We write $r_{t}=e^{\pi_{X}^{*} \varphi_{t}} r_{0}$ and $\tilde{r}_{t}=e^{\pi_{K}^{*} \psi_{t}} \tilde{r}_{0}$ with $\varphi_{t}$ a smooth curve in $\hat{\Xi}\left(X, J^{X}, \eta_{r_{0}}\right)^{\mathbb{T}}$ and $\psi_{t}=\hat{\boldsymbol{\Theta}}\left(\varphi_{t}\right)$ a curve in $\hat{\Xi}\left(K, J^{K}, \eta_{\tilde{r}_{0}}\right)^{\mathbb{T}}$. Let $\Pi_{X}=\left(r_{0}, \pi_{X}\right): V \cong \mathbb{R}^{+} \times N^{X}$ be the product structure on $V$ induced by $X$ and $r_{0}$ and similarly $\Pi_{K}=\left(r_{0}, \pi_{K}\right): V \cong \mathbb{R}^{+} \times N^{K}$. Since $N_{\tilde{r}_{t}}=N_{r_{t}}$, we have

$$
\left(\Pi_{K} \circ \Pi_{X}^{-1}\right)\left(e^{-\varphi_{t}(x)}, x\right)=\left(e^{-\psi_{t}\left(\Psi_{t}(x)\right)}, \Psi_{t}(x)\right),
$$

where $\Psi_{t}=\Psi_{r_{t}}^{K} \circ\left(\Psi_{r_{t}}^{X}\right)^{-1}: N^{X} \rightarrow N^{K}$. Since $\Pi_{K} \circ \Pi_{X}^{-1}$ is a diffeomorphism, $\Psi_{t}$ is a smooth curve of diffeomorphisms, hence $\psi_{t}$ and $\tilde{r}_{t}$ are also smooth curves.

To compute the derivative, by translation of the parameter $t$, it suffices to relate the functions $\Psi_{X}^{*} \dot{r}_{0}$ and $\Psi_{K}^{*} \dot{\tilde{r}}_{0}$ in $C_{N_{0}}^{\infty}(\mathbb{R})^{\mathbb{T}}$ where $N_{0}:=N_{r_{0}}=N_{\tilde{r}_{0}}, \Psi_{X}=\Psi_{r_{0}}^{X}$ and $\Psi_{K}=\Psi_{\tilde{r}_{0}}^{K}$. For any $p \in N_{0}$, we let

$$
\Phi_{t}(p):=\Phi_{\psi_{t}\left(\pi_{K}(p)\right)}^{-J K}(p)
$$


where $\Phi_{s}^{-J K}$ stands for the flow of $-J K$ on $V$. Now $\Phi_{t}(p) \in N_{r_{t}}=N_{\tilde{r}_{t}}$ and so, for any $p \in N_{0}$, we have

$$
\Phi_{\varphi_{t}\left(\pi_{X}\left(\Phi_{t}(p)\right)\right)}^{J X}\left(\Phi_{t}(p)\right) \in N_{0}
$$

where $\Phi_{s}^{J X}$ is the flow of $J X$ on $V$. Taking derivative at $t=0$ in (20), we thus obtain an element in $T_{p} N_{0}$. To compute this derivative, we use that

$$
\begin{gathered}
\left.\frac{d}{d t} \Phi_{t}(p)\right|_{t=0}=-\dot{\psi}_{0}\left(\pi_{K}(p)\right) J K_{p} \\
\varphi_{0}\left(\pi_{X}(q)\right)=0, \quad \Phi_{0}^{J X}=I d_{T V}
\end{gathered}
$$

and get

$$
\left.\frac{d}{d t}\left(\Phi_{\varphi_{t}\left(\pi_{X}\left(\Phi_{t}(p)\right)\right)}^{J X}\left(\Phi_{t}(p)\right)\right)\right|_{t=0}=\dot{\varphi}_{0}\left(\pi_{X}(p)\right) J X_{p}-\dot{\psi}_{0}\left(\pi_{K}(p)\right) J K_{p}
$$

As $\left(\mathcal{D}_{0}\right)_{p}=T_{p} V \cap J T_{p} V$, and $K_{p}$ and $X_{p}$ are in $T_{p} N_{0}$, we conclude that

$$
\dot{\varphi}_{0}\left(\pi_{X}(p)\right) X_{p}-\dot{\psi}_{0}\left(\pi_{K}(p)\right) K_{p} \in\left(\mathcal{D}_{0}\right)_{p},
$$

which, after acting with $\eta_{r_{0}}$, gives

$$
\dot{\psi}_{0} \circ \pi_{K}=\frac{\dot{\varphi}_{0} \circ \pi_{X}}{\eta_{r_{0}}(K)}
$$

on $N_{0}$. This means equivalently that $\Psi_{K}^{*} \dot{\tilde{r}}_{0}=\Psi_{X}^{*}\left(\dot{r}_{0} / \eta_{r_{0}}(K)\right)$ as required.

The formula for $\eta_{\tilde{r}_{t}}$ follows because by Lemma 2.5, $\Psi_{r_{t}}^{X} \circ\left(\Psi_{r_{t}}^{K}\right)^{-1}$ is a CR isomorphism between the CR structures induced by $\eta_{\tilde{r}_{t}}$ and $\eta_{r_{t}}$ on $N^{K}$ and $N^{X}$ respectively

It immediately follows that $\hat{\boldsymbol{\Theta}}$ is smooth, and its derivative is given as follows.

Corollary 2.18. Let $\varphi \in \hat{\Xi}\left(X, J^{X}, \eta_{0}^{X}\right)^{\mathbb{T}}$ and $\psi:=\hat{\Theta}(\varphi) \in \hat{\Xi}\left(K, J^{K}, \eta_{0}^{K}\right)^{\mathbb{T}}$ induce $C R$ structures $\left(\mathcal{D}_{\varphi}, J_{\varphi}\right)$ and $\left(\mathcal{D}_{\psi}, J_{\psi}\right)$ on $N^{X}$ and $N^{K}$ with contact forms $\eta_{\varphi}^{X}=\eta_{0}^{X}+d^{c} \varphi$ and $\eta_{\psi}^{K}=\eta_{0}^{K}+d^{c} \psi$ respectively. Then there is a CR isomorphism $\Psi_{\varphi}:\left(N^{X}, \mathcal{D}_{\varphi}, J_{\varphi}\right) \rightarrow$ $\left(N^{K}, \mathcal{D}_{\psi}, J_{\psi}\right)$ such that for any $\dot{\varphi} \in C_{N^{X}}^{\infty}(\mathbb{R})^{\mathbb{T}}$,

$$
\dot{\psi}:=d \hat{\boldsymbol{\Theta}}_{\varphi}(\dot{\varphi})=\Psi_{\varphi}^{*}\left(\frac{\dot{\varphi}}{\eta_{\varphi}^{X}(K)}\right) \quad \text { and } \quad \eta_{\psi}^{K}=\Psi_{\varphi}^{*}\left(\frac{\eta_{\varphi}^{X}}{\eta_{\varphi}^{X}(K)}\right) .
$$

\section{Extremal Sasaki metrics and Weighted extremal Kähler metrics}

3.1. Extremal Sasaki metrics. Let $(N, \mathcal{D}, J, X)$ be a Sasaki manifold. The Levi-Civita connection of (local) Sasaki-Reeb quotients by $X$ pulls back to a connection $\nabla^{X}$ on $\mathcal{D}$, preserving the transversal Kähler structure $\left(g_{X}, J, \omega_{X}\right)$, which turns out to be (see e.g. 222, $\S 4])$ the Tanaka-Webster connection [60] of $(\mathcal{D}, J, X)$. Thus the scalar curvature of SasakiReeb quotients pulls back to the Tanaka-Webster scalar curvature $\operatorname{Scal}\left(g_{X}\right)$ of $\nabla^{X}$.

Definition 3.1. [12] $(N, \mathcal{D}, J, X)$ is a $C S C$ or extremal Sasaki structure if $\operatorname{Scal}\left(g_{X}\right)$ is constant or is a transversal Killing potential, respectively. Equivalently any Sasaki-Reeb quotient with respect to $X$ is a CSC or extremal Kähler metric, respectively.

The Kähler geometry on Sasaki-Reeb quotients with respect to $X \in \mathfrak{c r}_{+}(N, \mathcal{D}, J)$, induced by an extremal Sasaki structure $(N, \mathcal{D}, J, K)$ with respect to a possibly different Sasaki-Reeb vector field $K \in \mathfrak{c r}_{+}(N, \mathcal{D}, J)^{X}$, was studied in [1].

Lemma 3.2. [1] Let $(M, J, g, \omega)$ be the Kähler orbifold corresponding to the Sasaki-Reeb quotient of $(N, \mathcal{D}, J)$ with respect to a quasiregular $X \in \mathfrak{c r}_{+}(N, \mathcal{D}, J)$, and $f=\eta_{\mathcal{D}}^{X}(K)>0$ the induced positive Killing potential on $(M, J, \omega)$ by $K \in \mathfrak{c r}_{+}(N, \mathcal{D}, J)^{X}$ via Lemma 1.4. Then $(\mathcal{D}, J, K)$ is an extremal Sasaki structure on $N$ if and only if the smooth function

$$
\left.\operatorname{Scal}_{f}(g):=f^{2} \operatorname{Scal}(g)-2(m+1) f \Delta_{g} f-(m+2)(m+1)\right)|d f|_{g}^{2}
$$


is a Killing potential on $(M, J, \omega)$.

When $K=X$ (i.e. $f=1$ ) we recover Definition 3.1. More generally, Lemma 3.2 allows us to reduce the study of extremal Sasaki metrics (with respect to a possibly irregular Sasaki-Reeb field $K$ ) in terms of a fixed Kähler manifold or orbifold $M$, obtained from a fixed regular or quasiregular Sasaki-Reeb field $X \in \mathfrak{c r}_{+}(N, \mathcal{D}, J)^{K}$. This is the point of view we take in this article.

Definition 3.3. Let $(g, \omega)$ be a Kähler metric on $(M, J)$ and $f$ be a Killing potential for $g$. We say that $g$ is $f$-extremal if its $f$-scalar curvature $\operatorname{Scal}_{f}(g)$, given by (21), is also a Killing potential.

Lemma 3.2 motivates the following definition.

Definition 3.4. [1] Let $(N, \mathcal{D}, J, X)$ be a Sasaki manifold, $K \in \mathfrak{c r}_{+}(N, \mathcal{D}, J)^{X}$ and $f=$ $\eta_{\mathcal{D}}^{X}(K)>0$. The $K$-scalar curvature $\operatorname{Scal}_{K}\left(g_{X}\right)$ of $(N, \mathcal{D}, J, X)$ is the function induced on $N$ by (21) on any Sasaki-Reeb quotient by $X$. We say $X$ is $K$-extremal if $\operatorname{Scal}_{K}\left(g_{X}\right)$ is a transversal Killing potential of $\left(g_{X}, J, \omega_{X}\right)$.

Thus Lemma 3.2 asserts that for $K \in \mathfrak{c r}_{+}(N, \mathcal{D}, J)^{X}, X$ is $K$-extremal if and only if $(\mathcal{D}, J, K)$ is extremal as in Definition 3.1. In particular, if $K=\lambda X$ with $\lambda>0$ constant, $K$-extremality reduces to extremality.

3.2. The Calabi problem for $f$-extremal Kähler metrics. As observed in [5, 28, 38], many features of the theory of extremal Kähler metrics extend naturally to the $f$-extremal case. In particular, one can formulate a weighted version of the Calabi problem [15] which seeks an $f$-extremal Kähler metric $(g, \omega)$ with $\omega \in \zeta$, a Kähler class. In this setting, we pin down the function $f$ indirectly by fixing first a quasiperiodic holomorphic vector field $\check{K}$ in the Lie algebra $\check{\mathfrak{t}}$ of a (compact) torus $\check{\mathbb{T}} \leqslant \operatorname{Aut}_{r}(M, J)$ inside the reduced group of automorphisms of $(M, J)$ (see e.g. [31]), and secondly, a real constant $\kappa>0$ such that for any $\check{\mathbb{T}}$-invariant Kähler metric $(g, \omega)$ with $\omega \in \zeta$, the Killing potential $f$ of $\check{K}$ with respect to $g$, normalized by $\int_{M} f \omega^{m} / m !=\kappa$, is positive on $M$, see [5, Lemma 1].

Problem 3.5. Is there a $\check{\mathbb{T}}$-invariant Kähler metric $(g, \omega)$ on $(M, J)$ with $\omega \in \zeta$, which is $f$-extremal, where $f$ is the Kähler potential of $\check{K}$ with respect to $g$ determined by $\int_{M} f \omega^{m} / m !=\kappa$ ? We refer to such metrics as $(\check{K}, \kappa)$-extremal.

As observed in [40], if $\left(g_{0}, \omega_{0}\right)$ and $(g, \omega)$ are two $\check{\mathbb{T}}$-invariant Kähler metrics in $\zeta$ with Kähler forms $\omega=\omega_{0}+d d^{c} \varphi$ for a $\check{\mathbb{T}}$-invariant smooth function $\varphi$ on $M$, then the corresponding $\kappa$-normalized Killing potentials $f$ and $f_{0}$ of $\check{K}$ are related by

$$
f=f_{0}+d^{c} \varphi(\check{K}) .
$$

Another useful observation from [28, 38,40$]$ is that when $M$ is compact, any $(\check{K}, \kappa)$ extremal metric on $(M, J)$ is invariant under a maximal torus in $\operatorname{Aut}_{r}(M, J)$, so we can assume without loss of generality that $\check{\mathbb{T}}$ itself is a maximal torus inside $\operatorname{Aut}_{r}(M, J)$.

3.3. The Calabi problem for $K$-extremal Sasaki metrics. In the case when $\left(M, J, \omega_{0}\right)$ is obtained as a Sasaki-Reeb quotient of a compact regular $\mathbb{T}$-invariant Sasaki manifold $\left(N, \mathcal{D}_{0}, J, X\right)$ with Sasaki-Reeb vector field $X \in \mathfrak{t}_{+}$and corresponding contact 1-form $\eta_{0}:=\eta_{\mathcal{D}_{0}}^{X}$, any other vector field $K \in \mathfrak{t}_{+}$pins down a positive Killing potential $f_{0}=\eta_{0}(K)$ for the induced Killing vector field

$$
\check{K}:=K+\langle X\rangle \in \operatorname{Lie}(\check{\mathbb{T}})=\mathfrak{t} / \mathbb{R} \cdot X
$$

on $\left(M, J, \omega_{0}\right)$, where $\check{\mathbb{T}}=\mathbb{T} / \mathbb{S}_{X}^{1}$ is the induced isometric torus action on $M$ with $\mathbb{S}_{X}^{1} \leqslant \mathbb{T}$ being the $\mathbb{S}^{1}$-action induced by $X$. By Lemma 1.17, any other compatible Sasaki structure 
$\eta=\eta_{0}+\alpha+d^{c} \varphi \in \mathcal{S}\left(X, J^{X}\right)^{\mathbb{T}}$ for $\alpha \in \Omega_{X, \mathrm{cl}}^{1}(N)^{\mathbb{T}}$ and $\varphi \in \hat{\Xi}\left(X, J^{X}, \eta_{0}^{X}\right)^{\mathbb{T}}$-see (2) - gives rise to a Killing potential $f:=\eta^{X}(K)$ of $\check{K}$ with respect to the induced Kähler structure $\omega_{\varphi}=\omega_{0}+d d^{c} \varphi$ on $(M, J)$, and $f$ and $f_{0}$ are related by (9), i.e., satisfy (22). It thus follows that the Killing potentials $f$ and $f_{0}$ are $\kappa$-normalized with

$$
\kappa:=\frac{1}{(2 \pi) m !} \int_{N} \eta^{X}(K) \eta^{X} \wedge\left(d \eta^{X}\right)^{m}
$$

In particular, $\kappa$ does not depend on $\eta^{X} \in \mathcal{S}\left(X, J^{X}\right)^{\mathbb{T}}$, a fact that can be also deduced from the $\mathbb{T}$-equivariant Gray-Moser argument, see Remark 1.8. This is consistent with Lemma 1.15 in which $K$ is seen as a positive affine-linear function on the natural moment polytope $P_{X} \subseteq \check{\mathfrak{t}}^{*}$ associated to $\left(N, \mathcal{D}_{0}, J, X\right)$.

Equivalently, we can think in terms of the induced polarization $L$ of $(M, J)$, as in Example 2.3. In this situation, the action of $\mathbb{T}$ on the cone $(V, J)$ corresponds to $\mathbb{T} \leqslant \operatorname{Aut}(M, L)$, and $\check{\mathbb{T}}=\mathbb{T} / \mathbb{S}^{1}$ is the induced action on $(M, J)$; furthermore, for a fixed $K \in \mathfrak{t}$, and any $\mathbb{T}$-invariant hermitian product $h$ on $L$ whose curvature form is a $\check{\mathbb{T}}$-invariant Kähler metric $\omega \in \zeta=2 \pi c_{1}(L)$, there is a naturally defined Killing potential $f$ of $\check{K}$. Furthermore, for any other $\mathbb{T}$-invariant hermitian product $e^{\varphi} h$ on $L$, the corresponding Killing potential for $\check{K}$ with respect to $\omega_{\varphi} \in \zeta$ is given by (22).

Definition 3.6. Since $K \in \mathfrak{t}$ determines $\check{K}$ and $\kappa$ in both the regular Sasaki and polarized cases, we shall also refer to a $(\check{K}, \kappa)$-extremal Kähler metric $\left(g_{\varphi}, \omega_{\varphi}\right)$ on $(M, J)$ in the Kähler class $\zeta=2 \pi c_{1}(L)$ as $K$-extremal.

Thus $K$-extremal metrics on $(M, J, \zeta)$ correspond to $K$-extremal Sasaki structures on $\left(N, X, J^{X}\right)$. We can sharpen this observation as follows.

Proposition 3.7. Let $\left(N, \mathcal{D}_{0}, J\right)$ be a compact $C R$ manifold of Sasaki type and $X, K \in$ $\mathfrak{c r}_{+}\left(N, \mathcal{D}_{0}, J\right)$ with $[X, K]=0$. Suppose $X$ is quasiregular with Sasaki-Reeb quotient the compact Kähler orbifold $(M, J, \omega)$. Then there is an extremal Sasaki structure in $\mathcal{S}\left(K, J^{K}\right)$ if and only if there is a K-extremal Kähler metric in the Kähler class [ $\omega]$.

Proof. Let $\mathbb{T} \leqslant \operatorname{Aut}\left(N, \mathcal{D}_{0}, J\right) \leqslant \operatorname{Aut}\left(N, X, J^{X}\right)$ be the (compact) torus generated by $X$ and $K$. Then there is $K$-extremal Kähler metric in the Kähler class $[\omega]$ if and only if there is a $K$-extremal Sasaki structure $\eta^{X}$ in $\mathcal{S}\left(X, J^{X}\right)^{\mathbb{T}}$. Since $K$-extremality of $\eta^{X}$ depends only on the transversal geometry, we may translate $\eta^{X}$ by any closed basic 1 -form $\alpha$, and hence this holds if and only if there is a $K$-extremal Sasaki structure in $\Xi\left(X, J^{X}, \eta_{\mathcal{D}_{0}}^{X}\right)^{\mathbb{T}}$. By Lemma 3.2 and the bijection $\hat{\boldsymbol{\Theta}}$ of (19), this holds if and only if there is an extremal Sasaki structure in $\Xi\left(K, J^{K}, \eta_{\mathcal{D}_{0}}^{K}\right)^{\mathbb{T}}$.

Hence it only remains to show that if there is an extremal Sasaki structure $\eta$ in $\mathcal{S}\left(K, J^{K}\right)$, then there is also one in $\Xi\left(K, J^{K}, \eta_{\mathcal{D}_{0}}^{K}\right)^{\mathbb{T}}$. Since (again) the extremality condition depends only on the transversal geometry, we may assume $\eta \in \Xi\left(K, J^{K}, \eta_{\mathcal{D}_{0}}^{K}\right)$. Now by [12, Theorem 4.8] $\eta$ is invariant under a maximal torus $\mathbb{T}_{\max } \leqslant \operatorname{Aut}(N, \mathcal{D}, J)^{K} \leqslant \operatorname{Aut}(V, J)^{K}$ (where $\mathcal{D}=\operatorname{ker} \eta$ ). Note that $\mathbb{T}_{\text {max }}$ is also maximal in $\operatorname{Aut}(V, J)^{K}$ : any torus containing $\mathbb{T}_{\max }$ defines an abelian subalgebra of $\mathfrak{a u t}(V, J)^{K}$, which descends to an abelian subalgebra of $\mathfrak{a u t}(V, J)^{K} /<K, J K>\leqslant \mathfrak{h}_{0}\left(N, K, J^{K}\right)$, and this cannot be larger than the Lie algebra of $\mathbb{T}_{\max }$ by [12. Since $\mathfrak{h}_{0}\left(N, K, J^{K}\right)$ is a finite dimensional Lie algebra, it follows that $\operatorname{Aut}(V, J)^{K}$ is a finite dimensional complex Lie group. Hence $\mathbb{T}_{\max }$ is conjugate to a maximal torus containing $\mathbb{T}$, and apply the conjugating automorphism to $\eta$, we obtain an extremal Sasaki structure in $\Xi\left(K, J^{K}, \eta_{\mathcal{D}_{0}}^{K}\right)^{\mathbb{T}}$. 


\section{Properness of the Weighted Mabuchi energy}

In this section, we consider a compact Sasaki manifold $(N, \mathcal{D}, J, K)$, with corresponding contact form $\eta_{0}^{K}$, and fix a maximal compact torus $\mathbb{T} \leqslant \operatorname{Aut}(N, \mathcal{D}, J)$ such that $K \in$ t. By [12, Theorem 4.8] (see the proof of Proposition [3.7), the search for an extremal Sasaki structure in $\mathcal{S}\left(K, J^{K}\right)$ can be reduced to the search of such structures in the space $\Xi\left(K, J^{K}, \eta_{0}^{K}\right)^{\mathbb{T}}$ of $\eta_{0}^{K}$-normalized, T-invariant Sasaki structures, see Definition 1.11. We denote by $K_{\text {ext }} \in \mathfrak{t}$ the extremal vector field associated to $(K, \mathbb{T})$, see Definition B.1 and [35], which is well-defined irrespective of the existence of an extremal Sasaki structures in $\Xi\left(K, J^{K}, \eta_{0}^{K}\right)^{\mathbb{T}}$, but which will coincide with the vector field defined by the transversal scalar curvature of any extremal Sasaki structure in that space, should it exist.

We fix a quasiregular Sasaki-Reeb vector field $X \in \mathfrak{t}_{+}$in the Reeb cone of $\left(N, K, J^{K}, \mathbb{T}\right)$. We consider the induced action of $\mathbb{T}$ and of its complexification $\mathbb{G}=\mathbb{T}_{\mathbb{C}}$ on the polarized complex cone $(V, J, K)$ (see Lemma 2.10) and identify the polarized complex cone of $(N, \mathcal{D}, J, X)$ with $(V, J, X)$, see Section 2, The important feature of this setting is that $\mathbb{G}$ naturally acts on the spaces $\mathcal{R}_{X}(V, J)^{\mathbb{T}}$ and $\mathcal{R}_{K}(V, J)^{\mathbb{T}}$ of $\mathbb{T}$-invariant cone potentials of $(V, J, X)$ and $(V, J, K)$, respectively. Using the basepoints $r_{0}^{X} \in \mathcal{R}_{X}(V, J)^{\mathbb{T}}$ and $r_{0}^{K} \in \mathcal{R}_{K}(V, J)^{\mathbb{T}}$ corresponding to the contact forms $\eta_{0}^{X}, \eta_{0}^{K}$, there is an induced action of $\mathbb{G}$ on the corresponding spaces $\hat{\Xi}\left(X, J^{X}, \eta_{0}^{X}\right)^{\mathbb{T}}$ and $\hat{\Xi}\left(K, J^{K}, \eta_{0}^{K}\right)^{\mathbb{T}}$ of $\mathbb{T}$-invariant Sasaki potentials.

Definition 4.1. (see e.g. [34, 35]) The (relative) Mabuchi energy is the functional

$$
\mathbf{M}^{K}: \hat{\Xi}\left(K, J^{K}, \eta_{0}^{K}\right)^{\mathbb{T}} \rightarrow \mathbb{R}
$$

characterized by

$$
\begin{aligned}
\left(d \mathbf{M}^{K}\right)_{\psi}(\dot{\psi}) & =\int_{N} \dot{\psi}\left(\operatorname{Scal}\left(g_{\psi}\right)-\eta_{\psi}\left(K_{\mathrm{ext}}\right)\right) \eta_{\psi} \wedge d \eta_{\psi}^{m}, \\
\mathbf{M}^{K}(0) & =0
\end{aligned}
$$

where for any $\psi \in \hat{\Xi}\left(K, J^{K}, \eta_{0}^{K}\right)^{\mathbb{T}}, \eta_{\psi}:=\eta_{0}^{K}+d_{K}^{c} \psi$ stands for the corresponding contact form in $\Xi\left(K, J^{K}, \eta_{0}^{K}\right)^{\mathbb{T}}$ whereas $\operatorname{Scal}\left(g_{\psi}\right)$ denotes the corresponding transversal scalar curvature.

Definition 4.2. The $K$-twisted (relative) Mabuchi energy is the functional

$$
\mathbf{M}_{K}^{X}: \hat{\Xi}\left(X, J^{X}, \eta_{0}^{X}\right)^{\mathbb{T}} \rightarrow \mathbb{R}
$$

characterized by

$$
\begin{aligned}
\left(d \mathbf{M}_{K}^{X}\right)_{\varphi}(\dot{\varphi}) & =\int_{N} \dot{\varphi}\left(S c a l_{K}\left(g_{\varphi}\right)-\eta_{\varphi}\left(K_{\mathrm{ext}}\right)\right)\left(\eta_{\varphi}(K)\right)^{-m-3} \eta_{\varphi} \wedge d \eta_{\varphi}^{m}, \\
\mathbf{M}_{K}^{X}(0) & =0 .
\end{aligned}
$$

Both functionals are invariant under additive constants, i.e., they descend to the spaces $\Xi\left(K, J^{K}, \eta_{0}^{K}\right)^{\mathbb{T}}$ and $\Xi\left(X, J^{X}, \eta_{0}^{X}\right)^{\mathbb{T}}$, respectively.

Remark 4.3. As $X \in \mathfrak{t}_{+}$is quasiregular, $\mathbf{M}_{K}^{X}$ gives rise to a functional $\mathbf{M}_{\check{K}, \kappa}$ acting on the space of $\check{\mathbb{T}}$-invariant (where we recall $\check{\mathbb{T}}=\mathbb{T} / \mathbb{S}_{X}^{1}$ ) $\omega_{0}$-relative Kähler potentials on the Sasaki-Reeb quotient $\left(M, J, \omega_{0}\right)$, defined by

$$
\left(d \mathbf{M}_{\check{K}, \kappa}\right)_{\varphi}(\dot{\varphi})=(2 \pi) \int_{M} \dot{\varphi}\left(\operatorname{Scal}_{f_{\varphi}}\left(\omega_{\varphi}\right)\right)^{\perp} f_{\varphi}^{-m-3} \omega_{\varphi}^{m}, \quad \mathbf{M}_{\check{K}, \kappa}(0)=0,
$$

where $\omega_{\varphi}=\omega_{0}+d d^{c} \varphi$ is the Kähler metric corresponding to $\varphi, f_{\varphi}$ is the $\kappa$-normalized Killing potential of $\check{K}$ with respect to $\omega_{\varphi}$, and $\left(\operatorname{Scal}_{f_{\varphi}}\left(\omega_{\varphi}\right)\right)^{\perp}$ denotes the orthogonal projection of the corresponding $f_{\varphi}$-scalar curvature to the orthogonal complement of the 
space of $\omega_{\varphi}$-Killing potentials of elements of Lie $(\check{\mathbb{T}})$, with respect to global $L^{2}$-product $\left(\phi_{1}, \phi_{2}\right)_{f_{\varphi}}=\int_{M} \phi_{1} \phi_{2} f_{\varphi}^{-m-3} \omega_{\varphi}^{m}$. This is, up to the multiplicative factor $2 \pi$, the relative weighted Mabuchi energy introduced in [39]. Equivalently, in terms of the momentum polytope $P_{X} \subseteq \check{\mathfrak{t}}^{*}$ of $\left(M, \omega_{0}, \check{\mathbb{T}}\right), \mathbf{M}_{K}^{X}$ is $2 \pi$ times the Mabuchi functional $\mathbf{M}_{v, w}$ defined in [40], where the weights $v(x)=\left(\ell_{K}(x)\right)^{-1-m}$ and $w(x)=\ell_{\text {ext }}(x) \ell_{K}(x)^{-3-m}$ are determined by the affine-linear functions $\ell_{K}(x)$ and $\ell_{\text {ext }}(x)$ on $\mathfrak{t}^{*}$, corresponding to $K, K_{\text {ext }} \in \mathfrak{t}$, see Lemma 1.15.

Lemma 4.4. Let $\hat{\boldsymbol{\Theta}}: \hat{\Xi}\left(X, J^{X}, \eta_{0}^{X}\right)^{\mathbb{T}} \rightarrow \hat{\Xi}\left(K, J^{K}, \eta_{0}^{K}\right)^{\mathbb{T}}$ be the bijection defined by (19); then $\mathbf{M}^{K} \circ \hat{\boldsymbol{\Theta}}=\mathbf{M}_{K}^{X}$.

Proof. We check $\mathbf{M}^{K} \circ \hat{\boldsymbol{\Theta}}$ satisfies the characterization of $\mathbf{M}_{K}^{X}$. Clearly $\mathbf{M}^{K}(\hat{\boldsymbol{\Theta}}(0))=$ $\mathbf{M}^{K}(0)=0$ and if $\varphi \in \hat{\Xi}\left(X, J^{X}, \eta_{0}^{X}\right)^{\mathbb{T}}$ with $\psi=\hat{\boldsymbol{\Theta}}(\varphi)$ then $d\left(\mathbf{M}^{K} \circ \hat{\boldsymbol{\Theta}}\right)_{\varphi}=\left(d \mathbf{M}^{K}\right)_{\psi} \circ(d \hat{\boldsymbol{\Theta}})_{\varphi}$. We now apply Corollary 2.18. As $\Psi_{\varphi}$ is a CR diffeomorphism between the CR structures induced by $\varphi$ and $\psi$, (46) implies that $\Psi_{\varphi}^{*}\left(\frac{\operatorname{Scal}_{K}\left(g_{\varphi}\right)}{\eta_{\varphi}(K)}\right)=\operatorname{Scal}\left(g_{\psi}\right)$, and the result follows.

We now recall the definition of the $d_{1}$-distance on the space $\hat{\Xi}\left(K, J^{K}, \eta_{0}^{K}\right)^{\mathbb{T}}$, originally due to Darvas [20] in the Kähler case, and extended by He-Li [35] to the Sasaki case.

Definition 4.5. For $\psi_{0}, \psi_{1} \in \hat{\Xi}\left(K, J^{K}, \eta_{0}^{K}\right)^{\mathbb{T}}$, we let

$$
d_{1}\left(\psi_{0}, \psi_{1}\right):=\inf _{\psi_{t}}\left\{\int_{0}^{1}\left(\int_{N}\left|\dot{\psi}_{t}\right| \eta_{\psi_{t}} \wedge d \eta_{\psi_{t}}^{m}\right) d t\right\}
$$

where the infimum is taken over all smooth paths $\psi_{t}, t \in[0,1]$, connecting $\psi_{0}$ and $\psi_{1}$ inside $\hat{\Xi}\left(K, J^{K}, \eta_{0}^{K}\right)^{\mathbb{T}}$.

The fact that $d_{1}$ is a distance is not obvious, and is one of the main results of [35]. We define the $d_{1}$-distance on the space $\hat{\Xi}\left(X, J^{X}, \eta_{0}^{X}\right)^{\mathbb{T}}$ by the same formula. When $X$ is regular, $d_{1}$ is, up to a factor $2 \pi$, the distance defined in [20] on the space of relative Kähler potentials on $(M, J)$. We now show that $\hat{\boldsymbol{\Theta}}$ is bilipschitz with respect to $d_{1}$.

Lemma 4.6. There exists $\Lambda \in \mathbb{R}^{+}$, depending only on $(N, \mathcal{D}, J, K, X)$, such that

$$
\frac{1}{\Lambda} d_{1}\left(\varphi_{0}, \varphi_{1}\right) \leq d_{1}\left(\hat{\boldsymbol{\Theta}}\left(\varphi_{0}\right), \hat{\boldsymbol{\Theta}}\left(\varphi_{1}\right)\right) \leq \Lambda d_{1}\left(\varphi_{0}, \varphi_{1}\right) .
$$

Proof. Let $\varphi_{t}$ be a smooth path connecting $\varphi_{0}$ and $\varphi_{1}$ in $\hat{\Xi}\left(K, J^{K}, \eta_{0}^{K}\right)^{\mathbb{T}}$. By Corollary 2.18, we have

$$
\int_{0}^{1}\left(\int_{N}\left|\dot{\psi}_{t}\right| \eta_{\psi_{t}} \wedge d \eta_{\psi_{t}}^{m}\right) d t=\int_{0}^{1}\left(\int_{N} \frac{\left|\dot{\varphi}_{t}\right|}{\left(\eta_{\varphi_{t}}(K)\right)^{m+2}} \eta_{\varphi_{t}} \wedge d \eta_{\varphi_{t}}^{m}\right) d t
$$

The key point is that the positive functions $f_{t}=\eta_{\varphi_{t}}(K)$ define $\kappa$-normalized Kähler potentials on $M$ with respect to the Kähler metrics $\omega_{\varphi_{t}}$, i.e., they are related by the formula (22). It thus follows (see e.g. [40, Lemma 1] for a general statement) that $f_{t}(N)=[a, b]$ is a fixed positive interval (independent of $\varphi_{0}, \varphi_{1}$ and $\varphi_{t}$ ), i.e., we have the uniform estimates

$$
\frac{1}{b^{m+2}} d_{1}\left(\varphi_{0}, \varphi_{1}\right) \leq d_{1}\left(\psi_{0}, \psi_{1}\right) \leq \frac{1}{a^{m+2}} d_{1}\left(\varphi_{0}, \varphi_{1}\right) .
$$

We next introduce a suitable notion of properness of $\mathbf{M}^{K}$ (resp. $\mathbf{M}_{K}^{X}$ ), analogous to the corresponding notions in Kähler geometry, see [21, 54, 62, To this end, we consider the action of $\mathbb{G}:=\mathbb{T}_{\mathbb{C}}$ on the spaces $\hat{\Xi}\left(X, J^{X}, \eta_{0}^{X}\right)^{\mathbb{T}}$ and $\hat{\Xi}\left(K, J^{K}, \eta_{0}^{K}\right)^{\mathbb{T}}$ as explained in the beginning of the section.

Lemma 4.7. $\mathbb{G}$ acts by $d_{1}$ isometries on $\hat{\Xi}\left(X, J^{X}, \eta_{0}^{X}\right)^{\mathbb{T}}$ and $\hat{\Xi}\left(K, J^{K}, \eta_{0}^{K}\right)^{\mathbb{T}}$. 
Proof. For any smooth curve $r(t)$ of cone potentials on $(V, J, X)$, and any $g \in \mathbb{G}$, let $\left(g^{*} r\right)(t)=r(t) \circ g$ be the corresponding $\mathbb{G}$ action on curves. We compute the $d_{1}$-lengths of $r(t)$ and $\left(g^{*} r\right)(t)$ :

$$
\begin{aligned}
\int_{0}^{1} \int_{N}\left|\frac{d\left(g^{*} r\right)(t)}{d t}\right|\left(\eta_{\left(g^{*} r\right)(t)} \wedge\left(d \eta_{\left(g^{*} r\right)(t)}\right)^{m}\right) d t & =\int_{0}^{1} \int_{N}\left|g^{*} \dot{r}(t)\right| g^{*}\left(\eta_{r(t)} \wedge\left(d \eta_{r(t)}\right)^{m}\right) d t \\
& =\int_{0}^{1} \int_{N}|\dot{r}(t)| \eta_{r(t)}\left(d \eta_{r(t)}\right)^{m} d t
\end{aligned}
$$

Lemma 4.8. $\hat{\Theta}: \hat{\Xi}\left(X, J^{X}, \eta_{0}^{X}\right)^{\mathbb{T}} \rightarrow \hat{\Xi}\left(K, J^{K}, \eta_{0}^{K}\right)^{\mathbb{T}}$ is $\mathbb{G}$-equivariant.

Proof. Obvious from the definition of $\hat{\boldsymbol{\Theta}}$.

The following crucial result is a refinement (in the regular case) of the uniqueness result of [57] (which in turn is a Sasaki version of [7]).

Lemma 4.9. Suppose $(N, \mathcal{D}, J, \mathbb{T}, X, K)$ is as above with $X$ regular. Then any two extremal Sasaki structures $\psi, \tilde{\psi} \in \hat{\Xi}\left(K, J^{K}, \eta_{0}^{K}\right)^{\mathbb{T}}$ are isometric under the action of $\mathbb{G}$.

Proof. Using Proposition 2.15 and the fact that $\hat{\boldsymbol{\Theta}}$ is $\mathbb{G}$-equivariant (Lemma 4.8), it is enough to establish the result for the corresponding $K$-extremal Sasaki structures in $\mathcal{S}\left(X, J^{X}\right)^{\mathbb{T}}$. As $X$ is regular, it is enough to show that the induced $\check{\mathbb{T}}$-invariant $(K, \kappa)$ extremal Kähler metrics $\omega$ and $\tilde{\omega}$ on $\left(M, J, \zeta, \check{\mathbb{T}}=\mathbb{T} / \mathbb{S}_{X}^{1}\right)$ are isometric by an element of $\check{\mathbb{T}}_{\mathbb{C}}$. Indeed, as $\check{\mathbb{T}}$ is a maximal torus in the reduced group of automorphisms $\operatorname{Aut}_{r}(M, J)$, and the induced extremal vector field $\check{K}_{\text {ext }}:=\operatorname{Jgrad}_{g} S c a l_{f}(g)$ is central in the Lie algebra of Killing fields of $(M, J, g, \omega)$, we must have $\check{K}_{\text {ext }} \in \check{\mathfrak{t}}$; it then follows by [40, Theorem B1] (see also [28, 38]) that the group $A u t_{r}^{\check{T}}(M, J)$ of reduced automorphisms of $(M, J)$, commuting with the $\check{\mathbb{T}}$-action, is a reductive Lie group. As $\check{\mathbb{T}}$ is simultaneously central and maximal, it follows that the identity component of $A u t_{r}^{\check{\mathbb{T}}}(M, J)$ is abelian and equal to $\check{\mathbb{T}}_{\mathbb{C}}$.

The result now follows from the uniqueness result in [41], established in the more general context of $(v, w)$-extremal Kähler metrics and building on an argument in [16], which implies that any two $\check{\mathbb{T}}$-invariant $(\check{K}, \kappa)$-extremal Kähler metrics in $\zeta$ are isometric by an element in the connected component of the identity of the group $A u t_{r}^{\overleftarrow{\mathbb{T}}}(M, J)$.

With this understood, for any $\psi_{0}, \psi_{1} \in \hat{\Xi}\left(K, J^{K}, \eta_{0}^{K}\right)^{\mathbb{T}}$, we let

$$
d_{1}^{\mathbb{G}}\left(\psi_{0}, \psi_{1}\right):=\inf _{g_{0}, g_{1} \in \mathbb{G}}\left\{d_{1}\left(g_{0} \cdot \psi_{0}, g_{1} \cdot \psi_{1}\right)\right\}=\inf _{g \in \mathbb{G}}\left\{d_{1}\left(\psi_{0}, g \cdot \psi_{1}\right)\right\} .
$$

and define similarly $d_{1}^{\mathbb{G}}\left(\varphi_{0}, \varphi_{1}\right)$ for $\varphi_{0}, \varphi_{1} \in \hat{\Xi}\left(X, J^{X}, \eta_{0}^{X}\right)^{\mathbb{T}}$.

Definition 4.10. $\mathbf{M}^{K}$ (resp. $\mathbf{M}_{K}^{X}$ ) is $\mathbb{G}$-proper if there exist constants $\Lambda>0$ and $C$, such that for any $\psi \in \hat{\Xi}\left(K, J^{K}, \eta_{0}^{K}\right)^{\mathbb{T}}$ we have $\mathbf{M}^{K}(\psi) \geq \Lambda d_{1}^{\mathbb{G}}(0, \psi)-C$ (resp. for any $\varphi \in \hat{\Xi}\left(X, J^{X}, \eta_{0}^{X}\right)^{\mathbb{T}}$ we have $\left.\mathbf{M}_{K}^{X}(\varphi) \geq \Lambda d_{1}^{\mathbb{G}}(0, \varphi)-C\right)$.

As $\hat{\boldsymbol{\Theta}}(0)=0$ by the definition of $\hat{\boldsymbol{\Theta}}$, Lemmas 4.4 and 4.6 yield

Corollary 4.11. $\mathbf{M}^{K}$ is $\mathbb{G}$-proper if and only if $\mathbf{M}_{K}^{X}$ is $\mathbb{G}$-proper.

The main result in this section extends one implication in [34, Theorem 1] and [35, Theorem 5.1].

Theorem 4.12. Let $(N, \mathcal{D}, J)$ be a compact $C R$ manifold of Sasaki type, $\mathbb{T} \leqslant \operatorname{Aut}(N, \mathcal{D}, J)$ a maximal torus, and $X, K \in \mathfrak{t}_{+}$with $X$ regular. If there exists an extremal Sasaki structure $\eta_{\psi}$ associated to $\psi \in \hat{\Xi}\left(K, J^{K}, \eta_{0}^{K}\right)^{\mathbb{T}}$, then $\mathbf{M}^{K}$ is $\mathbb{G}$-proper. 
Proof. One can directly check that in the setting above, all the conditions of the general existence/properness principle of [21, Section 3] hold true with

$$
(\mathcal{R}, d, F, G)=\left(\hat{\Xi}\left(X, J^{X}, \eta_{0}^{X}\right)^{\mathbb{T}}, d_{1}, \mathbf{M}^{K}, \mathbb{G}\right) .
$$

Indeed, for showing the conditions (A1)-(A4) of [21], we notice that the $d_{1}$ completion of $\hat{\Xi}\left(K, J^{K}, \eta_{0}^{K}\right)^{\mathbb{T}}$ and the extension of $\mathbf{M}^{K}$ is defined and proved in 35] whereas the fact that the $\mathbb{G}$-action is isometric is established in Lemma 4.7 above. For proving the conditions (P1)-(P7) of [21], we notice that: (P1) follows from [35, Theorem 6.7] and the fact that the (relative) Mabuchi energy $\mathbf{M}^{K}$ differs from the functional $\mathcal{K}$ in [35] by an affine-linear function on bounded geodesics, see [9, Prop. 10] or [41] for a direct argument with respect to $\mathbf{M}_{K}^{X}$ on the regular quotient; (P2) follows from [35, Theorem 6.6]; (P3) is the regularity result [34, Theorem 5.4] which can be extended from the CSC to the extremal case by the argument in [33]. (P4) and (P5) are established in Lemmas 4.7] and 4.9] above. (P6) follows from the general principle in [23, Section 6] (see in particular [23, Prop. 6.8]) whereas (P7) is automatically satisfied as it can be checked easily, see also [35, Prop. 5.1].

Remark 4.13. An alternative way to establish Theorem 4.12 is to compare directly the notion of $\mathbb{G}$-properness of $\mathbf{M}^{K}$ with the properness notion used in [34, Theorem 1] and [35, Theorem 5.1]. W. He considers in [34] the identity component $G:=A_{u t}\left(N, K, J^{K}\right)$ of the group of diffeomorphisms of $N$ preserving $K$ and the transversal holomorphic structure $J^{K}$, which naturally acts on the space $\mathcal{S}\left(K, J^{K}\right)$ of compatible contact forms by pullback. There is an induced action of $G$ on the quotient space $\mathcal{S}\left(K, J^{K}\right) / \Omega_{X, \mathrm{cl}}^{1}(N)$ and hence, by using the base point $\eta_{0}^{K}$, on the marking $\Xi\left(K, J^{K}, \eta_{0}^{K}\right)$. As $G$ does not act on the space of $\eta_{0}^{K}$-relative Sasaki potentials $\hat{\Xi}\left(K, J^{K}, \eta_{0}^{K}\right)$, a slice $\hat{\Xi}_{0}\left(K, J^{K}, \eta_{0}^{K}\right):=\left(\mathbf{I}^{K}\right)^{-1}(0)$ is introduced, where $\mathbf{I}^{K}: \hat{\Xi}\left(K, J^{K}, \eta_{0}^{K}\right) \rightarrow \mathbb{R}$ is a Sasaki version of the Aubin-Mabuchi functional defined by

$$
\begin{aligned}
\left(d \mathbf{I}^{K}\right)_{\psi}(\dot{\psi}) & =\int_{N} \dot{\psi} \eta_{\psi} \wedge d \eta_{\psi}^{m}, \\
\mathbf{I}^{K}(0) & =0 .
\end{aligned}
$$

Unlike $\mathbf{M}^{K}, \mathbf{I}^{K}$ is not invariant but is equivariant under additive real constants and thus determines a unique representative $\psi_{0} \in \hat{\Xi}_{0}\left(K, J^{K}, \eta_{0}^{K}\right)$ of $\eta_{\psi} \in \Xi\left(K, J^{K}, \eta_{0}^{K}\right)$. This leads to an action of $G$ on the slice $\hat{\Xi}_{0}\left(K, J^{K}, \eta_{0}^{K}\right)$, which we denote by $[g] \cdot \psi_{0}$, where $[g] \in[G]$, the effective quotient of $G$. One direction of the statements in [34, Theorem 1] and [35, Theorem 5.1] then yields that if there exists an extremal Sasaki metric in $\mathcal{S}\left(K, J^{K}\right)^{\mathbb{T}}$, the Mabuchi energy $\mathbf{M}^{K}$ is proper on $\hat{\Xi}\left(K, J^{K}, \eta_{0}^{K}\right)^{\mathbb{T}}$ with respect to $[G]$, i.e., satisfies

$$
\mathbf{M}^{K}(\psi) \geq \Lambda d_{1}^{[G]}\left(0, \psi_{0}\right)-C
$$

where $d_{1}^{[G]}\left(0, \psi_{0}\right):=\inf _{g \in G}\left\{d_{1}\left(0,[g] \cdot \psi_{0}\right)\right\}$.

Now $\mathbb{G}$ also acts on $\mathcal{S}\left(K, J^{K}\right)^{\mathbb{T}}$, hence on $\hat{\Xi}_{0}\left(K, J^{K}, \eta_{0}^{K}\right)$, and we let $[\mathbb{G}]$ denote the effective quotient. Although $\mathbb{G}$ is not a subgroup of $G,[\mathbb{G}]$ is a subgroup of $[G]$ and the $\mathbb{G}$-orbits of induced Sasaki structures are inside the $[G]$-orbits on $\hat{\Xi}_{0}\left(K, J^{K}, \eta_{0}^{K}\right)$. On the other hand, the action of $\mathbb{G}$ on $\hat{\Xi}\left(K, J^{K}, \eta_{0}^{K}\right)^{\mathbb{T}}$ includes translations by constants, so we conclude that for any $\psi \in \hat{\Xi}\left(K, J^{K}, \eta_{0}^{K}\right)^{\mathbb{T}}$ with projection $\psi_{0}$ to $\hat{\Xi}_{0}\left(K, J^{K}, \eta_{0}^{K}\right)$ we have

$$
d_{1}^{[\mathbb{G}]}\left(0, \psi_{0}\right):=\inf _{g \in \mathbb{G}}\left\{d_{1}\left(0,[g] \cdot \psi_{0}\right)\right\} \geq d_{1}^{[G]}\left(0, \psi_{0}\right), \quad d_{1}^{\mathbb{G}}(0, \psi)=d_{1}^{\mathbb{G}}\left(0, \psi_{0}\right) \leq d_{1}^{[\mathbb{G}]}\left(0, \psi_{0}\right) .
$$

It thus follows that the conclusion of Theorem 4.12 will hold true if one can improve (23) by replacing $G$ with $[\mathbb{G}]$ for functions $\psi \in \hat{\Xi}\left(K, J^{K}, \eta_{0}^{K}\right)^{\mathbb{T}}$. An inspection of the arguments in [34] (which in turn are an application of the general principle of [21]) reveals that the only missing ingredient to do so is proving that the $[\mathbb{G}]$-action is transitive on the space of 
smooth extremal Sasaki potentials $\psi_{0} \in \hat{\Xi}_{0}\left(K, J^{K}, \eta_{0}^{K}\right)^{\mathbb{T}}$. This property is established in Lemma 4.9 above.

Note that this argument yields the stronger notion of $d_{1}^{[\mathbb{G}]}$-properness of $\mathbf{M}^{K}$, should an extremal Sasaki metric in $\mathcal{S}\left(K, J^{K}\right)$ exist. However, as the map $\hat{\boldsymbol{\Theta}}$ does not respect the corresponding slices $\hat{\Xi}_{0}\left(K, J^{K}, \eta_{0}^{K}\right)^{\mathbb{T}}=\left(\mathbf{I}^{K}\right)^{-1}(0)$ and $\hat{\Xi}_{0}\left(X, J^{X}, \eta_{0}^{X}\right)^{\mathbb{T}}=\left(\mathbf{I}^{X}\right)^{-1}(0)$, it is not immediately clear whether or not the energy $\mathbf{M}_{K}^{X}$ is also $d_{1}^{[\mathbb{G}]}$-proper. The somewhat weaker but more natural notion of $d_{1}^{\mathbb{G}}$-properness we use above remedies this, see Corollary 4.11,

\section{Weighted K-Stability of Regular Sasaki-Reeb quotients}

In this section we relate the existence of extremal Sasaki structures on a compact Sasaki manifold of regular type with a special case of the weighted K-stability of a polarized variety, defined in [40]. We thus assume that $(N, \mathcal{D}, J)$ is a compact CR manifold of Sasaki type, $\mathbb{T} \leqslant \operatorname{Aut}(N, \mathcal{D}, J)$ a torus, and $X \in \mathfrak{t}_{+}$a regular Sasaki-Reeb vector field. The Sasaki-Reeb quotient $(M, J, L)$ is a smooth projective polarized variety, as in Example 1.3. We denote by $\mathbb{T}$ the induced torus action on $L$, and by $\check{\mathbb{T}}=\mathbb{T} / \mathbb{S}_{X}^{1}$ the torus action on $(M, J)$.

5.1. Polarized test configurations. We first recall the notion of a (compactified) polarized test configuration associated to $(M, J, L, \mathbb{T})$, going back to [24, 55].

Definition 5.1. A $\mathbb{T}$-equivariant polarized test configuration of exponent $s \in \mathbb{N}$ associated to $(M, J, L, \mathbb{T})$ is a normal polarized variety $(\mathscr{M}, \mathscr{L})$ endowed an action $\mathbb{T} \leqslant \operatorname{Aut}(\mathscr{M}, \mathscr{L})$, and

- a (flat) surjective morphism $\pi: \mathscr{M} \rightarrow \mathbb{C} P^{1}$ such that $\check{\mathbb{T}}$ preserves each fibre $M_{t}:=\pi^{-1}(t)$ and, for $t \neq 0,\left(M_{t}, L_{t}:=\mathscr{L}_{\mid M_{t}}\right)$ is $\mathbb{T}$-equivariantly isomorphic, as a polarized variety, to $(M, L, \mathbb{T})$

- a $\mathbb{C}^{\times}$-action $\rho$ on $(\mathscr{M}, \mathscr{L})$ commuting with $\mathbb{T}$ and covering the usual $\mathbb{C}^{\times}$-action on $\mathbb{C} P^{1}$;

- an isomorphism of polarized varieties

$$
\lambda:\left(M \times\left(\mathbb{C} P^{1} \backslash\{0\}\right), L^{s} \otimes \mathcal{O}_{\mathbb{C} P^{1}}(s)\right) \cong\left(\mathscr{M} \backslash M_{0}, \mathscr{L}\right),
$$

which is equivariant with respect to the actions of $\mathbb{T} \times \mathbb{S}_{0}^{1}$ on $\left(M \times\left(\mathbb{C} P^{1} \backslash\{0\}\right), L^{s} \otimes\right.$ $\left.\mathcal{O}_{\mathbb{C} P^{1}}(s)\right)$ and $\mathbb{T} \times \mathbb{S}_{\rho}^{1}$ on $\left(\mathscr{M} \backslash M_{0}, \mathscr{L}\right)$, where $\mathbb{S}_{0}^{1}$ stands for the standard $\mathbb{S}^{1}$-action on $\mathcal{O}(1) \rightarrow \mathbb{C} P^{1}$ and $\mathbb{S}_{\rho}^{1}$ is the $\mathbb{S}^{1}$-action induced by $\rho$.

The test configuration $(\mathscr{M}, \mathscr{L})$ as above is called product if $\left(\mathscr{M} \backslash M_{\infty}, \pi\right)$ is $\check{\mathbb{T}}$-equivariantly isomorphic (as morphism of complex varieties) to $\left(M \times\left(\mathbb{C} P^{1} \backslash\{\infty\}\right), \pi_{\mathbb{C} P^{1}}\right)$.

Remark 5.2. Polarized test configurations are often introduced (see e.g. [48, 59]) only by the corresponding requirements for the induced torus actions on $\mathscr{M}, M$ and $\mathbb{C} P^{1}$, i.e., are independent of the chosen lifts of these actions to $\mathscr{L}$ and $L$. In particular, as $\mathcal{O}(1)$ is trivial over $\mathbb{C} P^{1} \backslash\{0\}$, the third condition reduces to the existence of a biholomorphism $\check{\lambda}: M \times\left(\mathbb{C} P^{1} \backslash\{0\}\right) \rightarrow \mathscr{M} \backslash M_{0}$ which is equivariant with respect to the induced actions of $\check{\mathbb{T}} \times \check{\mathbb{S}}_{0}^{1}$ on $M \times\left(\mathbb{C} P^{1} \backslash\{0\}\right)$ and $\check{\mathbb{T}} \times \mathbb{S}_{\check{\rho}}^{1}$ on $\mathscr{M} \backslash M_{0}$. It is not hard to see that given such a biholomorphism $\check{\lambda}$ (and assuming $L$ and $\mathscr{L}$ are very ample) one can always lift $\check{\rho}$, $\check{\mathbb{T}}$ and $\check{\lambda}$ to obtain a polarized test configuration in the sense of Definition 5.1 .

We shall further assume that the $\mathbb{T}$-equivariant polarized test configuration $(\mathscr{M}, \mathscr{L}, \pi, \rho)$ for $(M, J, L)$ as above has a smooth total space $\mathscr{M}$, and take $s=1$ for simplicity. We consider the associated polarized complex cones $(\mathscr{V}, X)$ and $(V, X)$, where $\mathscr{V}=\left(\mathscr{L}^{*}\right)^{\times}, V=$ $\left(L^{*}\right)^{\times}$and $X$ denotes the regular Sasaki-Reeb vector field corresponding to the fibrewise 
$\mathbb{S}^{1}$-actions on $\mathscr{L}$ and $L$ (see Example 2.3). According to Lemma 6.5, the induced momentum polytopes for the $\check{\mathbb{T}}$-actions on $\mathscr{M}$ and $M$ are equal to the transversal polytope $P_{X}$ defined by (8). By Lemma 1.15, $K \in \mathfrak{t}_{+}$is determined by an affine-linear function $\ell_{K}(x)$ on $\check{\mathfrak{t}}^{*}$, which is positive on $P_{X}$, whereas the extremal vector field $K_{\text {ext }}$ gives rise to an affine-linear function $\ell_{\text {ext }}(x)$ on $\check{\mathfrak{t}}^{*}$.

For a $\check{\mathbb{T}}$-invariant Kähler metric $\tilde{\Omega} \in 2 \pi c_{1}(\mathscr{L})$, we let $m_{\tilde{\Omega}}$ denote the $\check{\mathbb{T}}$-momentum map sending $\mathscr{M}$ to $P_{X}$, and set

$$
\begin{aligned}
\mathscr{F}_{K}^{\mathrm{ext}}(\mathscr{M}, \mathscr{L}):= & -\int_{\mathscr{M}}\left(\operatorname{Scal}_{\tilde{f}, m+2}(\tilde{\Omega})-\ell_{\mathrm{ext}}\left(m_{\tilde{\Omega}}\right)\right) \tilde{f}^{-3-m} \frac{\tilde{\Omega}^{m+1}}{(m+1) !} \\
& +2 \int_{\mathscr{M}} \tilde{f}^{-1-m}\left(\pi^{*} \omega_{F S}\right) \wedge \frac{\tilde{\Omega}^{m}}{m !}
\end{aligned}
$$

where $\omega_{\mathrm{FS}}$ is a Fubini-Study metric with $\operatorname{Ric}\left(\omega_{\mathrm{FS}}\right)=\omega_{\mathrm{FS}}, \tilde{f}=\ell_{K}\left(m_{\tilde{\Omega}}\right)$ is a Killing potential of the Killing vector field induced by $K$ on $\mathscr{M}$, and we have set

$$
S c a l_{f, \nu}(g):=f^{2} \operatorname{Scal}(g)-2(\nu-1) f \Delta_{g} f-\nu(\nu-1)|d f|_{g}^{2},
$$

as in [2].

Formula (24) is in fact the weighted Futaki invariant of Lahdilli [40] with weights $v(x)=$ $\ell_{K}(x)^{-m-1}$ and $w(x)=\ell_{\text {ext }}(x) \ell_{K}^{-m-3}(x)$; it follows that it is independent of the choice of $\tilde{\Omega} \in 2 \pi c_{1}(\mathscr{L})$. We also notice that the contact Futaki invariant associated to $(N, \mathcal{D}, K)$ (see Definition B.1) vanishes if and only if $K_{\text {ext }}=c_{K} K$, i.e., $\ell_{\text {ext }}(x)=c_{K} \ell_{K}(x)$, in which case we have $\mathscr{F}_{K}^{\operatorname{ext}}(\mathscr{M}, \mathscr{L})=\mathscr{F}_{K}(\mathscr{M}, \mathscr{L})$, where we have set

$$
\begin{aligned}
\mathscr{F}_{K}(\mathscr{M}, \mathscr{L}):= & -\int_{\mathscr{M}}\left(\operatorname{Scal}_{\tilde{f}, m+2}(\tilde{\Omega})-c_{K} \tilde{f}\right) \tilde{f}^{-3-m} \frac{\tilde{\Omega}^{m+1}}{(m+1) !} \\
& +2 \int_{\mathscr{M}} \tilde{f}^{-1-m}\left(\pi^{*} \omega_{F S}\right) \wedge \frac{\tilde{\Omega}^{m}}{m !} .
\end{aligned}
$$

Proposition 3.7 and [40, Theorem 2] then yield the following necessary condition for the existence of extremal Sasaki structures in $\mathcal{S}\left(K, J^{K}\right)$, which we refer to as relative $K$ weighted $\mathrm{K}$-semistability with respect to $\mathbb{T}$.

Theorem 5.3. Let $(N, \mathcal{D}, J, X, K)$ be as in Theorem 4.12. If there exists an extremal Sasaki structure in $\mathcal{S}\left(K, J^{K}\right)$, then we have $\mathscr{F}_{K}^{\mathrm{ext}}(\mathscr{M}, \mathscr{L}) \geq 0$ for any $\mathbb{T}$-equivariant smooth polarized test configuration with reduced central fibre $(\mathscr{M}, \mathscr{L})$ associated to $(M, J, L)$.

We can sharpen this to a positivity result as follows.

Lemma 5.4. Let $(M, J, L)$ a smooth polarized complex manifold, $\mathbb{T}$ be a maximal torus in $\operatorname{Aut}(M, L)$, and $\omega_{0} \in 2 \pi c_{1}(L)$ a $\check{\mathbb{T}}$-invariant Kähler metric, where $\check{\mathbb{T}} \leqslant \operatorname{Aut}_{r}(M, J)$ is the induced torus action on $M$. Suppose that $\mathbf{M}_{\breve{K}, \kappa}$ is $\mathbb{G}$-proper with respect to the action of $\mathbb{G}=\mathbb{T}_{\mathbb{C}}$ on $\check{\mathbb{T}}$-invariant $\omega_{0}$-relative Kähler potentials, induced by its natural action on hermitian metrics on $L$. Then, for any $\mathbb{T}$-equivariant smooth polarized test configuration $(\mathscr{M}, \mathscr{L})$ associated to $(M, J, L)$, which has reduced central fibre $\left(M_{0}, L_{0}\right)$ and is not a product, we have

$$
\mathscr{F}_{K}^{\operatorname{ext}}(\mathscr{M}, \mathscr{L})>0 \text {. }
$$

Proof. The $\mathbb{G}$-properness implies that $\mathbf{M}_{\check{K}, \kappa}$ is bounded from below on the space of $\check{\mathbb{T}}$ invariant $\omega_{0}$-relative Kähler potentials, so that by [40, Thm. 7], we have

$$
\mathscr{F}_{\bar{K}}^{\operatorname{ext}}(\mathscr{M}, \mathscr{L}) \geq 0
$$

We thus have to establish that if $\mathscr{F}_{K}^{\text {ext }}(\mathscr{M}, \mathscr{L})=0$ for a smooth polarized test configuration $(\mathscr{M}, \mathscr{L}, \mathbb{T})$ with reduced central fibre associated to $(M, J, L, \mathbb{T})$, then $(\mathscr{M}, \mathscr{L}, \mathbb{T})$ must be 
a $\mathbb{C}^{\times}$-equivariant compactification at infinity of the product $\mathbb{C} \times M$. To this end, we use a slight modification of the arguments from [8, [52]: suppose that $\mathscr{F}_{K}^{\operatorname{ext}}(\mathscr{M}, \mathscr{L})=0$ and denote by $u_{t}$ the smooth ray of $\check{T}$-invariant $\omega_{0}$-relative Kähler potentials on $(M, J, \omega, \check{\mathbb{T}})$ with $u_{0}=0$ (defined by the $\mathbb{C}^{\times}$-action on $\mathbb{T}$-invariant hermitian products on $\mathscr{L} \rightarrow \mathscr{M}$ on the test configuration $(\mathscr{M}, \mathscr{L}, \mathbb{T})$ ). Using that

$$
0=\mathscr{F}_{K}^{\operatorname{ext}}(\mathscr{M}, \mathscr{L})=\lim _{t \rightarrow+\infty} \frac{\mathbf{M}_{\check{K}, \kappa}\left(u_{t}\right)}{t}
$$

(see again [40, Thm. 7]) and the $\mathbb{G}$-properness of $\mathbf{M}_{\check{K}, \kappa}$, we conclude that

$$
0 \leq d_{1}^{\mathbb{G}}\left(0, u_{t}\right) \leq o(t) .
$$

To any test configuration $(\mathscr{M}, \mathscr{L})$ as above, and a $\check{\mathbb{T}}$-invariant $\omega_{0}$-relative Kähler potential $\varphi_{0}$, Phong-Sturm [30] associate a $C^{1, \overline{1}}$ geodesic ray $\varphi_{t}$ emanating from $\varphi_{0}$. A key estimate in [8] reads

$$
\left|\varphi_{t}-u_{t}\right| \leq C
$$

which implies $d_{1}\left(u_{t}, \varphi_{t}\right) \leq C$. As $\mathbb{G}$ acts by $d_{1}$-isometries (Lemma 4.7) and using the triangle inequality, we have

$$
d_{1}^{\mathbb{G}}\left(0, \varphi_{t}\right)-C \leq d_{1}^{\mathbb{G}}\left(0, u_{t}\right) .
$$

and also

$$
0 \leq d_{1}^{\mathbb{G}}\left(\varphi_{0}, \varphi_{t}\right) \leq o(t) .
$$

In our case, we can take $\varphi_{0}=0$ (as we consider the space $\mathbb{T}$-invariant hermitian metrics $h=e^{-2 \varphi} h_{0}$ on $L$ with $\mathbb{T}$ being a maximal torus in $\left.\operatorname{Aut}(M, L)\right)$. In [21] and 8] the authors rather consider $\varphi_{0}$ to be the normalized potential of a CSC metric in order to insure that it is fixed under a maximal compact subgroup of $K \leqslant A u t_{r}(M, J)$, but we do not need this assumption as we apply [21, Prop. 6.8] with $K:=\mathbb{T}$ and $G:=\mathbb{T}_{\mathbb{C}}=\mathbb{G}$ acting on the space $\check{T}$-invariant $\omega_{0}$-relative Kähler potentials. One property needed for this result that we did not find readily available in the literature is that for a vector field $Y \in \mathfrak{t}$ on $L$, the flow $\Phi_{t}^{-J Y} \cdot \varphi$ generates a smooth geodesic in the space of $\breve{\mathbb{T}}$-invariant potentials. This is classically known to be true for the induced action $\left[\Phi_{t}^{-J Y}\right] \cdot \varphi$ on normalized relative Kähler potentials with respect to the Aubin-Mabuchi functional I, i.e., such that $\mathbf{I}\left(\left[\Phi_{t}^{-J Y}\right] \cdot \varphi\right)=0$ (see e.g. [31]), but the argument in [8, Lemma 4.2] actually shows that $\Phi_{t}^{-J Y} \cdot \varphi$ differs from $\left[\Phi_{t}^{-J Y}\right] \cdot \varphi$ by an affine linear function in $t$.

Now, the arguments in [8, Lemma 4.1] and [52, Prop. 4.10] (we have a $o(t)$ bound for $d_{1}^{\mathbb{G}}\left(\varphi_{0}, \varphi_{t}\right)$ as in [52]) extend tautologically in our situation, and show that $\varphi_{t}$ is induced by the flow of a vector field $J Y$ on $L$ with $Y \in \mathfrak{t}$. Finally, the fact that $(\mathscr{M}, \mathscr{L})$ is a product test configuration follows from [8, Prop. 4.3] or [52, Thm. A.6].

This lemma now leads to the following main result, which is an improvement of Theorem 5.3 to the following statement, which we call relative $K$-weighted $\mathrm{K}$-stability with respect to $\mathbb{T}$.

Theorem 5.5. Let $(N, \mathcal{D}, J, X)$ be a compact regular Sasaki manifold, $\mathbb{T}$ a maximal torus in $\operatorname{Aut}(N, \mathcal{D}, J)$, and $K \in \mathfrak{t}_{+}$a Sasaki-Reeb vector field such that there exists an extremal Sasaki structure in $\mathcal{S}\left(K, J^{K}\right)$. Denote by $(M, J, L, \mathbb{T})$ the smooth polarized variety obtained as Sasaki-Reeb quotient with respect to $X$, with the induced action of $\mathbb{T}$ on $L$. Then, for any $\mathbb{T}$-equivariant smooth polarized test configuration $(\mathscr{M}, \mathscr{L})$ associated to $(M, J, L)$, which has reduced central fibre $\left(M_{0}, L_{0}\right)$ and is not a product, we have $\mathscr{F}_{K}^{\operatorname{ext}}(\mathscr{M}, \mathscr{L})>0$.

Proof. By Theorem 4.12, the (modified) Mabuchi energy $\mathbf{M}^{K}$ is $\mathbb{G}$-proper and by Corollary 4.11 , the corresponding $K$-twisted modified Mabuchi energy $\mathbf{M}_{K}^{X}$ is also $\mathbb{G}$-proper. 
Recall that $\mathbb{G}$-action was introduced by the natural action of $\mathbb{T}_{\mathbb{C}}$ on the space of cone potentials $\mathcal{R}_{X}(V, J)$, Equivalently, in terms of the smooth polarized variety $(M, J, L, \mathbb{T})$, the group $\mathbb{G}=\mathbb{T}_{\mathbb{C}}$ acts naturally on the space of $\mathbb{T}$-invariant hermitian metrics $h$ on $L$ and, therefore, by considering the corresponding curvatures, on the space of $\check{\mathbb{T}}$-invariant Kähler metrics $\omega \in 2 \pi c_{1}(L)$. Furthermore, by introducing a basepoint $h_{0}$ (with curvature $\omega_{0}$ ), $\mathbb{G}$ acts also on the space of $\check{\mathbb{T}}$-invariant $\omega_{0}$-relative Kähler potentials (which is naturally identified with the space $\hat{\Xi}\left(X, J^{X}, \eta_{0}^{X}\right)^{\mathbb{T}}$ of $\mathbb{T}$-invariant Sasaki potentials). As we recalled in Remark 4.3, the $K$-twisted Mabuchi energy $\mathbf{M}_{K}^{X}$ defined on the space $\hat{\Xi}\left(X, J^{X}, \eta_{0}^{X}\right)^{\mathbb{T}}$ corresponds to the relative $(\check{K}, \kappa)$-Mabuchi energy $\mathbf{M}_{\check{K}, \kappa}$ acting on the space of $\check{\mathbb{T}}$-invariant $\omega_{0}$-relative Kähler potentials $\varphi$ of Kähler metrics $\omega_{\varphi}=\omega_{0}+d d^{c} \varphi \in 2 \pi c_{1}(L)$.

By Theorem 4.12, $\mathbf{M}_{\breve{K}, \kappa}$ is $\mathbb{G}$-proper in the sense that for any $\check{\mathbb{T}}$-invariant $\omega_{0}$-relative Kähler potential $\varphi$

$$
\mathbf{M}_{K, \kappa}(\varphi) \geq \Lambda d_{1}^{\mathbb{G}}(0, \varphi)-C
$$

where we recall $d_{1}^{\mathbb{G}}:=\inf _{g \in \mathbb{G}} d_{1}(0, g \cdot \varphi)$. The result now follows from Lemma 5.4 below.

Remark 5.6. One can extend the conclusions of Theorems 4.12 and 5.5 to smooth polarized test configurations with reduced central fibre, which are $\mathbb{T}$-equivariant with respect to the torus $\mathbb{T} \leqslant \operatorname{Aut}(M, L)$ generated by the Sasaki-Reeb vector fields $X, K$, and the CR vector field $K_{\text {ext }}$ of Definition B.1. To this end, one needs to consider instead of $\mathbb{G}=\mathbb{T}_{\mathbb{C}}$, the action of the centralizer $G_{\mathbb{T}}$ of $\mathbb{T}$ in $\operatorname{Aut}(M, L)$ (which also acts on the spaces $\mathcal{R}_{X}(V, J)$ and $\mathcal{R}_{K}(V, J)$ ), and use the uniqueness of $\mathbb{T}$-invariant $K$-extremal metrics modulo $G_{\mathbb{T}}$ (which is established [41]) in order to obtain the properness of $\mathbf{M}_{\check{K}, \kappa}$ with respect to $G_{\mathbb{T}}$.

5.2. Transcendental Kähler test configurations. A key feature of Lahdilli's construction [40] is that it allows one to extend the conclusion of Theorem 5.3 to a more general class of smooth test configurations, the Kähler test configurations introduced in [23, 51], which are defined as follows

Definition 5.7. A 苂-equivariant Kähler test configuration associated to the Kähler manifold $(M, J)$ with a fixed Kähler class $\zeta$ is a smooth Kähler manifold $\mathscr{M}$, endowed a Kähler class $\mathscr{A}$ and a torus $\check{\mathbb{T}} \leqslant A u t_{r}(\mathscr{M})$ in its reduced group of automorphisms, and

- a surjective holomorphic map $\pi: \mathscr{M} \rightarrow \mathbb{C} P^{1}$ such that $\check{\mathbb{T}}$ preserves each fibre $M_{t}:=$ $\pi^{-1}(t)$ and, for $t \neq 0,\left(M_{t}, \zeta_{t}:=\mathscr{A}_{\mid M_{t}}\right)$ is $\check{\mathbb{T}}$-equivariantly isomorphic to $(M, \zeta, \check{\mathbb{T}})$;

- a $\mathbb{C}^{\times}$-action $\rho$ commuting with $\check{\mathbb{T}}$ and covering the usual $\mathbb{C}^{\times}$-action $\rho_{0}$ on $\mathbb{C} P^{1}$;

- a biholomorphism

$$
\lambda:\left(M \times\left(\mathbb{C} P^{1} \backslash\{0\}\right), \zeta\right) \cong\left(\mathscr{M} \backslash M_{0}, \mathscr{A}\right),
$$

which is equivariant with respect to the actions of $\check{\mathbb{T}} \times \mathbb{S}_{\rho_{0}}^{1}$ on $M \times\left(\mathbb{C} P^{1} \backslash\{0\}\right)$ and $\check{\mathbb{T}} \times \mathbb{S}_{\rho}^{1}$ on $\mathscr{M} \backslash M_{0}$.

Even in the case when $\zeta=2 \pi c_{1}(L)$ for some polarization $L$ of $(M, J)$, and $\mathscr{M}$ is a smooth projective manifold, the Kähler class $\mathscr{A}$ on $\mathscr{M}$ need not be integral, thus allowing for a stronger K-stability condition. Indeed, following [40], if we have a smooth, $\check{\mathbb{T}}$-equivariant test configuration $(\mathscr{M}, \mathscr{A})$ associated to $(M, J, L)$ as above, we can still use formula (24) in order to introduce the numerical invariant $\mathscr{F}_{K}^{\operatorname{ext}}(\mathscr{M}, \mathscr{A}$ ) (which again is independent of the choice of a $\check{\mathbb{T}}$-invariant Kähler metric $\tilde{\Omega} \in \mathscr{A}$ ). Furthermore, according to [40, Theorem 2], $\mathscr{F}_{K}^{\operatorname{ext}}(\mathscr{M}, \mathscr{A}) \geq 0$ for any $\check{\mathbb{T}}$-equivariant smooth Kähler test configuration with reduced central fibre $(\mathscr{M}, \mathscr{A})$ of $\left(M, J, 2 \pi c_{1}(L)\right)$, should an extremal Sasaki structure exist in $\mathcal{S}\left(K, J^{K}\right)$, i.e., Theorem 5.3 holds true even for smooth Kähler test configurations with reduced central fibre associated to $\left(M, J, 2 \pi c_{1}(L), \check{\mathbb{T}}\right)$.

We expect that the following will hold true. 
Conjecture 5.8. In the setting of Theorem 5.5, for any $\check{\mathbb{T}}$-equivariant smooth Kähler test configuration $(\mathscr{M}, \mathscr{A})$ associated to $\left(M, J, 2 \pi c_{1}(L)\right)$, which has a reduced central fibre and is not a product, we have $\mathscr{F}_{K}^{\operatorname{ext}}(\mathscr{M}, \mathscr{A})>0$.

Remark 5.9. In the light of recent results in the (unweighted) Kähler setting [52], and the discussion at the end of Remark 4.13, the main missing point for establishing the above conjecture is the improvement of the $\mathbb{G}$-properness of $\mathbf{M}_{K}^{X}$ to the stronger notion of $[\mathbb{G}]$-properness.

5.3. Transcendental Kähler classes. We make here a few comments about the obstruction theory for the existence of a $(\check{K}, \kappa)$-extremal Kähler metric on a general compact Kähler manifold $(M, J, \zeta)$. When $\zeta=2 \pi(L)$ for an ample line bundle, and taking $\check{\mathbb{T}} \leqslant A_{u t}(M, J)$ be a maximal torus with $\check{K} \in \check{\mathfrak{t}}$, this is covered by Theorem 5.5 and Conjecture 5.8 (by using Proposition [3.7). Following [40], one can extend the weighted K-stability notion to the general Kähler case. To this end, one fixes a momentum polytope $P \subseteq \check{\mathfrak{t}}^{*}$ for the $\check{\mathbb{T}}$-action on $(M, J, \zeta)$, which in turn gives rise to a positive affine-linear function $\ell(x)$ on $P$, such that the $\kappa$-normalized Killing potential $f$ of $\check{K}$ with respect to a $\check{\mathbb{T}}$-invariant Kähler metric $\omega \in \zeta$ is written as $f=\ell\left(m_{\omega}\right)$, where the momentum map $m_{\omega}$ of $(M, \omega, \check{\mathbb{T}})$ is normalized by $m_{\omega}(M)=P$. Furthermore, as shown in [40], the $L^{2}$ projection $\operatorname{Scal}_{f}(g)^{\text {ext }}$ of the $f$-scalar curvature $\operatorname{Scal}_{f}(g)$ of $\omega$ to the space of Killing potentials with respect to $\omega$ of elements of $\check{\mathfrak{t}}^{*}$, by using the weighted product $\left(\phi_{1}, \phi_{2}\right)_{f}=\int_{M} \phi_{1} \phi_{2} f^{-m-3} \omega^{m} / m$ ! can be written as $\operatorname{Scal}_{f}(g)^{\text {ext }}=\ell_{\text {ext }}\left(m_{\omega}\right)$ for an affine-linear function on $P$, independent of $\omega \in \zeta$. For any smooth $\check{\mathbb{T}}$-equivariant Kähler test configuration $(\mathscr{M}, \mathscr{A})$ associated to $(M, \zeta, \check{\mathbb{T}})$, and $\check{\mathbb{T}}$-invariant Kähler metric $\tilde{\Omega} \in \mathscr{A}$, one can still define a numerical invariant via formula (24), where the momentum map $m_{\tilde{\Omega}}$ is normalized by the condition $m_{\tilde{\Omega}}(\mathscr{M})=P$, and $\tilde{f}=\ell\left(m_{\tilde{\Omega}}\right)$. This expression is independent of the $\check{\mathbb{T}}$-invariant Kähler metric $\tilde{\Omega} \in \mathscr{A}$ according to [40]; furthermore, it is not hard to see that the numerical invariant does not depend on the chosen momentum polytope $P$ for $(M, \zeta, \check{\mathbb{T}})$ : it merely depends upon the data $(\check{K}, \kappa, \check{\mathbb{T}})$ so we shall denote it by $\mathscr{F}_{(\check{K}, \kappa)}^{\operatorname{ext}}(\mathscr{M}, \mathscr{A})$. In this more general Kähler setting, [41, Theorem 2] implies that if $\zeta$ admits a $(\check{K}, \kappa)$-extremal Kähler metric, then $\mathscr{F} \underset{K, \kappa}{\operatorname{ext}}(\mathscr{M}, \mathscr{A}) \geq 0$ for any smooth $\check{\mathbb{T}}$-equivariant test configuration with reduced central fibre associated to $(M, J, \zeta, \breve{\mathbb{T}})$, and we can extend Conjecture 5.8 to a relative $(\check{K}, \kappa)$-weighted K-stability statement.

\section{SAsaki K-Stability versus Weighted K-Stability}

6.1. Sasaki K-stability. Given a complex polarized cone $(V, J, K, \mathbb{T})$ as defined in Definition [2.2, it is known that (see e.g. the proof of [58, Theorem 3.1]) there exists a $\mathbb{T}$ equivariant holomorphic embedding $V \hookrightarrow \mathbb{C}^{N}$ sending $V$ biholomorphically to $\widehat{V} \backslash\{0\}$,

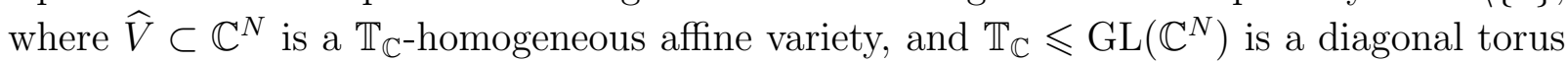
whose real form is identified with $\mathbb{T}$. When $K$ is quasiregular, one can further identify the space $\mathscr{H}$ of holomorphic functions on $V$ introduced in $\$ 2.2$ with the space of regular function functions on $\widehat{V}$ (see Remark 2.12), i.e., $\mathscr{H}=\mathbb{C}\left[x_{1}, \ldots, x_{N}\right] / I$ for a $\mathbb{T}_{\mathbb{C}}$-homogeneous ideal $I$. Thus, in the decomposition (16),,$\Gamma \subseteq \mathfrak{t}^{*}$ is the set of weights of the $\mathbb{T}_{\mathbb{C}}$-action on the space of regular functions. Given a polarization $K \in \mathfrak{t}_{+}$, the pair $(\widehat{V}, K)$ is referred to as a polarized affine cone.

In this latter setting, Collins and Székelyhidi [18] introduced the $\mathbb{T}$-equivariant index character

$$
F(K, t):=\sum_{\alpha \in \Gamma} e^{-t \alpha(K)} \operatorname{dim} \mathscr{H}_{\alpha}, \quad \operatorname{Re}(t)>0
$$


and prove (cf. [18, Prop. 4.3]) that $F(K, t)$ is a well-defined function in $t$, which has meromorphic extension to a neighbourhood of the origin, with Laurent expansion

$$
F(K, t)=\frac{a_{0}(K) m !}{t^{m+1}}+\frac{a_{1}(K)(m-1) !}{t^{m}}+O\left(t^{1-m}\right) .
$$

According to [18, Theorem 4.10], the coefficients $a_{0}(K)>0$ and $a_{1}(K)$ are smooth functions on $\mathfrak{t}_{+}$. With these ingredients, the following definition is proposed in [18].

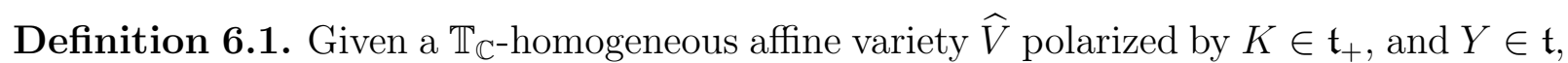
the $K$-Donaldson-Futaki invariant is defined to be the quantity

$$
\mathrm{DF}_{\widehat{V}, K}(Y):=\frac{a_{0}(K)}{m} D_{Y}\left(a_{1}(K) / a_{0}(K)\right)+\frac{a_{1}(K) D_{Y} a_{0}(K)}{m(m+1) a_{0}(K)},
$$

where $D_{Y}$ denotes the derivative in the direction of $Y$ on $\mathfrak{t}$.

If we assume, furthermore, that $(V, J)$ is smooth and $K$ is primitive in the lattice of circle subgroups of $\mathbb{T}$, then the quotient of $V$ by the subgroup $\mathbb{C}_{K}^{\times}$generated by $K$ is a polarized orbifold $\left(M_{K}, L_{K}\right)$ whose generic isotropy groups are trivial, and for which the orbifold Riemann-Roch formula of [50, (2.17)] gives

$$
\operatorname{dim}_{\mathbb{C}} H^{0}\left(M_{K}, L_{K}^{k}\right)=a_{0}(K) k^{n}+\left(a_{1}(K)+\rho(K)\right) k^{n-1}+\cdots
$$

where $\rho(K)$ is a periodic function in $k$ with average 0 , and, up to multiplicative positive dimensional constants, $a_{0}(K)$ and $a_{1}(K)$ are respectively the total volume and total scalar curvature of the polarized orbifold $\left(M_{K}, L_{K}\right)$. It follows that, up to a positive dimensional constant, $\mathrm{DF}_{\widehat{V}, K}(Y)$ coincides with the Futaki invariant of the real holomorphic vector field induced by $Y$ on the polarized orbifold $\left(M_{K}, L_{K}\right)$, see [18, Props. $4.3 \& 4.13$ and Theorem 4.14].

Remark 6.2. In the notation of Appendix $\mathrm{B}$, there is thus a dimensional constant $c(m)>0$ such that

$$
\operatorname{DF}_{\widehat{V}, K}(Y)=c(m) \operatorname{Fut}_{K}(Y),
$$

where $\operatorname{Fut}_{K}(Y)$ denotes the contact Futaki invariant $\operatorname{Fut}_{K}(Y)$ introduced in Definition B.1. Indeed by Remark B.2, $\mathrm{DF}_{\widehat{V}, K}(Y)$ and $\operatorname{Fut}_{K}(Y)$ agree up to a positive dimensional constant when $K$ is a quasiregular. However they are both continuous and homogeneous of order $-m$ in $K$ (where $m+1$ is the complex dimension of $\widehat{V}$ ) and the quasiregular directions are dense in $\mathfrak{t}_{+}$.

Following [18], Definition 6.1 can be used to assign a numerical invariant to any $\mathbb{T}$ equivariant test configuration associated to the polarized affine cone $(\widehat{V}, K)$, as follows. Let

$$
w=\left(w_{1}, \ldots, w_{N}\right), w_{i} \in \mathbb{Z}
$$

be a set of integers and denote by $\rho_{w}: \mathbb{C}^{\times} \rightarrow G L\left(\mathbb{C}^{N}\right)$ the weighted $\mathbb{C}^{\times}$-action defined by $w$. Taking the flat limit across $0 \in \mathbb{C}^{\times}$of the $\mathbb{C}^{\times}$orbit of $\widehat{V}$ under the action of $\rho_{w}$, we obtain a flat family of affine schemes over $\mathbb{C}$, with central fibre $C_{0}$. Besides the $\mathbb{T}$-action on $\widehat{V}_{0}$, the central fibre inherits the $\mathbb{C}^{\times}$-action $\rho_{w}$, and we denote by $Y_{w}$ the generator of the corresponding $\mathbb{S}^{1}$-action on $\mathbb{C}^{k}$ whose flow preserves $\widehat{V}_{0}$. It is shown in [18, Section 5] that for a fixed $K \in \mathfrak{t}_{+}$, Definition 6.1 can be applied on the scheme $\widehat{V}_{0}$ and gives a necessary condition for the existence of a CSC Sasaki structures (known as K-semistability).

Theorem 6.3. [18] Let $(N, \mathcal{D}, J)$ be a compact $C R$ manifold of Sasaki type, $\mathbb{T}$ a torus in $\operatorname{Aut}(N, \mathcal{D}, J)$, and $K \in \mathfrak{t}_{+}$a Sasaki-Reeb vector field such that $N$ admits a CSC Sasaki structure in $\mathcal{S}\left(K, J^{K}\right)$. Then, for any $\mathbb{T}$-equivariant test-configuration of the associated polarized affine cone $(\widehat{V}, K)$ with central fibre $\widehat{V}_{0}, \mathrm{DF}_{\widehat{V}_{0}, K}\left(Y_{w}\right) \geq 0$. 
6.2. Test configurations of polarized complex cones. The Definition 5.1 of a $\mathbb{T}$ equivariant polarized test configuration of a regular Sasaki-Reeb quotient $(M, J, L)$ provides a natural link to the theory of test configurations of affine cones described above. Indeed, given a $\mathbb{T}$-equivariant polarized test configuration $(\mathscr{M}, \mathscr{L}, \pi, \rho)$ for $(M, J, L)$ as in Definition 5.1, and assuming that $s=1$ and $\mathscr{M}$ is smooth for simplicity, we consider the corresponding complex cones $\mathscr{V}:=\left(\mathscr{L}^{*}\right)^{\times}$and $V:=\left(L^{*}\right)^{\times}$and denote by $\tilde{K}$ and $K$ the corresponding (regular) cone polarizations, see Examples 1.3 and 2.3 . We thus get the following objects:

(i) a flat surjective morphism $\pi: \mathscr{V} \rightarrow \mathbb{C} P^{1}$ such that $\mathbb{T}$ preserves each fibre $V_{t}:=\pi^{-1}(t)$ and, for $t \neq 0,\left(V_{t}, \tilde{K}_{\left.\right|_{V_{t}}}\right)$ is $\mathbb{T}$-equivariantly isomorphic, as a polarized complex cone, to $(V, K)$;

(ii) a $\mathbb{C}^{\times}$-action $\rho$ on $\mathscr{V}$ commuting with $\mathbb{T}$ and covering the usual $\mathbb{C}^{\times}$-action on $\mathbb{C} P^{1}$;

(iii) a biholomorphism

$$
\lambda: V \times\left(\mathbb{C} P^{1} \backslash\{0\}\right) \cong \mathscr{V} \backslash V_{0},
$$

which is equivariant with respect to the actions of $\mathbb{T} \times \mathbb{S}_{0}^{1}$ on $V \times\left(\mathbb{C} P^{1} \backslash\{0\}\right)$ and $\mathbb{T} \times \mathbb{S}_{\rho}^{1}$ on $\mathscr{V} \backslash V_{0}$, where $\mathbb{S}_{0}^{1}$ stands for the standard $\mathbb{S}^{1}$-action on $\mathcal{O}(1) \rightarrow \mathbb{C} P^{1}$.

This prompts the following

Definition 6.4. Let $(V, J, K)$ be a smooth polarized complex cone endowed with a holomorphic action of a compact torus $\mathbb{T}$, and $K \in \mathfrak{t}_{+}$a Sasaki-Reeb polarization. A smooth $\mathbb{T}$-equivariant test configuration of $(V, J, K)$ is a smooth polarized complex cone $(\mathscr{V}, \mathscr{J}, \tilde{K})$, endowed with a $\mathbb{T}$-action such that $\tilde{K} \in \tilde{\mathfrak{t}}^{+}$, satisfying the conditions (i)-(iii) above.

Note that Definition 6.4 does not require $K$ to be regular nor the existence of a regular Sasaki-Reeb vector field $X$ commuting with $K$. To simplify the notation, we shall at times omit the complex structures $J$ and $\mathscr{J}$.

Lemma 6.5. Let $(\mathscr{V}, \tilde{K})$ be a $\mathbb{T}$-equivariant smooth test configuration of polarized cones associated to $(V, K)$. Then, the reduced Sasaki-Reeb cone $\mathfrak{t}_{+}$of $(V, K)$ coincides with the reduced Sasaki-Reeb cone $\tilde{\mathfrak{t}}_{+}$of $(\mathscr{V}, \tilde{K})$. Moreover, $\tilde{K}=K \in \mathfrak{t}_{+}$.

Proof. For $t \neq 0$ we denote $\iota_{t}: V \rightarrow V_{t}$ a $\mathbb{T} \times \mathbb{R}^{+}$-equivariant biholomorphism given by the condition (i). By Lemma 1.15 and (7), the Reeb cone $\tilde{\mathfrak{t}}_{+} \subseteq \mathfrak{t}$ is independent of the choice of a compatible $\mathbb{T}$-invariant cone potential $\tilde{r} \in \mathcal{R}_{\tilde{K}}(\mathscr{V})^{\mathbb{T}}$. The same argument implies that the moment cone $\Sigma_{t}$ associated to $\left(V, K, \iota_{t}^{*} \tilde{r}\right)$ does not depend on $t$. We denote this cone by $\Sigma$. Using (17) we define the cone momentum maps $\mu^{t}: V \rightarrow \mathfrak{t}^{*}$ and $\tilde{\mu}: \mathscr{V} \rightarrow \mathfrak{t}^{*}$ associated respectively to the cones $\left(V, K, \iota_{t}^{*} \tilde{r}\right)$ and $(\mathscr{V}, \tilde{K}, \tilde{r})$. Since condition (i) ensures that orbits of $\mathbb{T}$ lie in the fibre $V_{t}$ of $\pi$ we have, for $p \in V_{t}$,

$$
\left\langle\mu_{p}^{t}, Y\right\rangle=\frac{1}{4} \iota_{t}^{*} d^{c}\left(\tilde{r}^{2}\right)(Y)=\frac{1}{4} d^{c}\left(\tilde{r}^{2}\right)(Y)=\left\langle\tilde{\mu}_{p}, Y\right\rangle .
$$

In particular, $\tilde{\mu}\left(\mathscr{V} \backslash V_{0}\right) \subseteq \Sigma$ and thus $\tilde{\mu}(\mathscr{V})=\Sigma$ because they are both strictly convex polyhedral cones as recalled in $\$ 1.3$. The relation (7) concludes the proof of the first claim whereas the condition (i) yields the second claim.

Consequently, we will identify the Sasaki-Reeb vector fields $\tilde{K}$ and $K$ and the reduced Sasaki-Reeb cones $\mathfrak{t}_{+}$and $\tilde{\mathfrak{t}}_{+}$in Definition 6.4 and in what follows.

Example 6.6. (Product test configuration of polarized cones) A particular class of smooth test configurations of polarized complex cones are the product test configurations. These are defined (similarly to Definition 5.1) by requiring that the holomorphic surjection $\left(\mathscr{V} \backslash V_{\infty}, \pi\right)$ is $\mathbb{T}$-equivariantly isomorphic to the product $\left(V \times\left(\mathbb{C} P^{1} \backslash\{\infty\}\right), \pi_{\mathbb{C} P^{1}}\right)$. In the regular case, examples are obtained by taking the polarized complex cones over product polarized test configurations of the regular quotient in the sense of [10, Example 2.8]. 


\subsection{The global Futaki invariant of a smooth test configuration of polarized complex cones.}

Definition 6.7. Given a smooth $\mathbb{T}$-equivariant test configuration $(\mathscr{V}, K)$ of the polarized complex cone $(V, K)$ with $K \in \mathfrak{t}_{+}$as in Definition 6.4, and a cone potential $\tilde{r} \in \mathcal{R}_{K}(\mathscr{V})^{\mathbb{T}}$, we let

$$
\begin{aligned}
\mathscr{G}_{K}(\mathscr{V}):= & -\frac{1}{(m+1) !} \int_{\mathscr{N}}\left(\operatorname{Scal}\left(\tilde{g}_{K}\right)-c_{K}\right) \tilde{\eta}_{\mathscr{D}}^{K} \wedge\left(d \tilde{\eta}_{\mathscr{D}}^{K}\right)^{m+1} \\
& +\frac{2}{m !} \int_{\mathscr{N}}\left(\pi^{*} \omega_{F S}\right) \wedge \tilde{\eta}_{\mathcal{D}}^{K} \wedge\left(d \tilde{\eta}_{\mathcal{D}}^{K}\right)^{m}
\end{aligned}
$$

where $(\mathscr{N}, \mathscr{D}, \mathscr{J}, K)$ is the induced Sasaki manifold with contact form $\tilde{\eta}_{\mathscr{D}}^{K}, c_{K}$ is the constant (48) of $N$, and $\omega_{\mathrm{FS}}$ is the Fubini-Study metric on $\mathbb{C} P^{1}$ satisfying $\operatorname{Ric}\left(\omega_{F S}\right)=\omega_{\mathrm{FS}}$. We shall refer to (30) as the global Futaki invariant of $(\mathscr{V}, K)$.

Note that, as shown for instance in [29], the first line of (30) is independent of the choice of a cone potential $\tilde{r} \in \mathcal{R}_{K}(\mathscr{V})^{\mathbb{T}}$ on $(\mathscr{V}, K)$ and so is the second line by a similar argument.

Proposition 6.8. Let $(\mathscr{V}, K)$ be a smooth $\mathbb{T}$-invariant test configuration of polarized complex cones associated to $(V, K)$ with $K \in \mathfrak{t}_{+}$. Consider the corresponding polarized affine cone $(\widehat{\mathscr{V}}, K)$ with central fibre the polarized affine variety $\left(\widehat{V}_{0}, K\right)$ and associated Donaldson-Futaki invariant $\mathrm{DF}_{\widehat{V}_{0}, K}\left(Y_{w}\right)$. Then

$$
\mathrm{DF}_{\widehat{V}_{0}, K}\left(Y_{w}\right)=\lambda(m) \mathscr{G} \mathscr{F}_{K}(\mathscr{V})
$$

for a dimensional constant $\lambda(m)>0$.

Proof. As both functionals in (31) depend continuously on $K \in \mathfrak{t}_{+}$(see [18, Theorem 3]) and are homogeneous of degree $-(m+1)$ it will be enough to prove (31) for $K$ primitive in the lattice of circle subgroups of $\mathbb{T}$ ( since the set of such vectors is dense in $\mathfrak{t}_{+} / \mathbb{R}^{+}$).

Assume that $K$ is a primitive element in the lattice of circle subgroups of $\mathbb{T}$, and denote $\mathbb{C}_{K}^{\times} \leqslant \mathbb{T}_{\mathbb{C}}$ the subgroup generated by $K$. The $\mathbb{C}_{K}^{\times}$GIT reduction of $\mathscr{V}$ can be identified (cf. [49, 50]) with the compact polarized orbifold $(\mathscr{M}, \mathscr{L})$ associated to the grading induced by $\mathbb{C}_{K}^{\times}$-action on the coordinates ring of the affine cone $\widehat{\mathscr{V}} \subseteq \mathbb{C}^{N}$, which also gives an orbifold embedding of $(\mathscr{M}, \mathscr{L})$ into a weighted projective space. By construction, $\mathscr{M} \simeq$ $\widehat{\mathscr{V}} \backslash\{0\} / \mathbb{C}_{K}^{\times} \simeq \mathscr{V} / \mathbb{C}_{K}^{\times}$, so that $\mathscr{M}$ has only cyclic orbifold singularities. The fact that $K$ is primitive is used to determine the orbifold structure of the quotient such that the generic

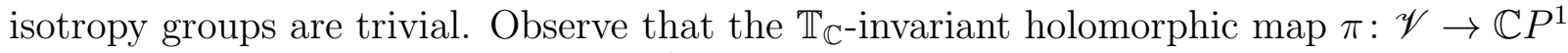
descends to the quotient $\check{\pi}: \mathscr{M} \rightarrow \mathbb{C} P^{1}$ as a $\mathbb{C}_{\rho}^{\times}$-equivariant surjective orbifold holomorphic map. Then one can define a complex orbifold $M:=V_{1} / \mathbb{C}_{K}^{\times} \simeq V / \mathbb{C}_{K}^{\times}$with orbiline bundle $L=\mathscr{L}_{\left.\right|_{M}}$ (the restriction of $L$ to the orbifold charts is taken so that we get a locally ample orbiline bundle in the sense of [50]). This orbiline bundle is in fact ample (in the sense of [50]) because $\left(\left(L^{*}\right)^{\times}, K\right) \simeq\left(V_{1}, K\right) \subseteq \widehat{\mathscr{V}}$ and sections of $\mathscr{L}$ restrict to section of $L$ over $M$. Finally, we observe that $(\mathscr{M}, \mathscr{L})$ defines a test configuration for $(M, L)$ in the sense of [50], as the map $\lambda$ in (29) (which is $\mathbb{T} \times \mathbb{C}_{\rho}^{\times}$equivariant) descends to a $\left(\mathbb{T} / \mathbb{S}_{K}^{1}\right) \times \mathbb{C}_{\rho}^{\times}$-equivariant map.

Using Chern-Weil theory on orbifolds (in particular that $c_{1}^{\text {orb }}(L)=\frac{1}{2 \pi}\left[\omega_{K}\right]$ for a 2 -form $\omega_{K}$ which pulls back as the differential of the connection 1 -form $\eta_{\mathcal{D}}^{K}$ on the $\mathbb{S}^{1}$ - bundle 
$N \rightarrow M)$, we have

$$
\begin{aligned}
\frac{1}{(2 \pi)} \mathscr{G}_{\mathscr{F}}(\mathscr{V})=\frac{2(2 \pi)^{m+1}}{m !} & \left\{\left(\mathscr{K}_{\mathscr{M} / \mathbb{C} P^{1}}^{\text {orb }} \cdot \mathscr{L}^{m}\right)_{\mathscr{M}}+\frac{c_{K}}{2(m+1)}\left(\mathscr{L}^{m+1}\right)_{\mathscr{M}}\right\} \\
=\frac{2(2 \pi)^{m+1}}{m !}\{ & \left(\mathscr{K}_{\mathscr{M} / \mathbb{C} P^{1}}^{\text {orb }} \cdot \mathscr{L}^{m}\right)_{\mathscr{M}} \\
& \left.\quad-\frac{m}{m+1}\left(\frac{\left(K_{M}^{\text {orb }} \cdot L^{m-1}\right)_{M}}{\left(L^{m}\right)_{M}}\right)\left(\mathscr{L}^{m+1}\right)_{\mathscr{M}}\right\}
\end{aligned}
$$

where $\mathscr{K}^{\text {orb }}$ and $K^{\text {orb }}$ stand for the (relative) canonical orbi-bundles on $\mathscr{M}$ and $M$, respectively, and the products are taken by using integration of forms over orbifolds. Notice that up to a positive dimensional constant, (32) is an orbifold version of the intersection formula for the Donaldson-Futaki invariant found by Odaka [48] and Wang [59] in the settings of projective schemes.

We next argue that, similarly to the conclusions of [48, 59], (32) computes, up to a positive dimensional constant, the orbifold version of the Donaldson-Futaki invariant associated to the central fibre $\left(M_{0}:=\check{\pi}^{-1}(0), L_{0}:=\mathscr{L}_{M_{0}}\right)$ of $(\mathscr{M}, \mathscr{L})$, defined in [50, Def. 6.4]. This follows from the considerations in [25, pp. 315] 2, namely that for $k \gg 0$ we have exact sequences

$$
\begin{aligned}
& 0 \rightarrow H^{0}\left(\mathscr{M}, \mathscr{L}^{k} \otimes \check{\pi}^{*} \mathcal{O}(-1)\right) \rightarrow H^{0}\left(\mathscr{M}, \mathscr{L}^{k}\right) \rightarrow H^{0}\left(M_{0}, L_{0}^{k}\right) \rightarrow 0 \\
& 0 \rightarrow H^{0}\left(\mathscr{M}, \mathscr{L}^{k} \otimes \check{\pi}^{*} \mathcal{O}(-1)\right) \rightarrow H^{0}\left(\mathscr{M}, \mathscr{L}^{k}\right) \rightarrow H^{0}\left(M, L^{k}\right) \rightarrow 0
\end{aligned}
$$

given by multiplication by the sections of $\mathcal{O}(-1)$ defining $M_{0}$ and $M_{\infty}$, respectively. This implies that $d_{k}:=\operatorname{dim}_{\mathbb{C}} H^{0}\left(M_{0}, L_{0}^{k}\right)=\operatorname{dim}_{\mathbb{C}} H^{0}\left(M, L^{k}\right)$ whereas the weight $w_{k}$ for the induced $\mathbb{C}^{\times}$-action on $H^{0}\left(M_{0}, L_{0}^{k}\right)$ is

$$
w_{k}=\operatorname{dim}_{\mathbb{C}} H^{0}\left(\mathscr{M}, \mathscr{L}^{k} \otimes \check{\pi}^{*} \mathcal{O}(-1)_{\mathbb{C} P^{1}}\right)=\operatorname{dim}_{\mathbb{C}}\left(\mathscr{M}, \mathscr{L}^{k}\right)-\operatorname{dim}_{\mathbb{C}} H^{0}\left(M, L^{k}\right) .
$$

Now the claim follows from the definition [50, Def. 6.4] and an easy computation using the orbifold Riemann-Roch formula (28).

To conclude the proof of the proposition, we use that $\mathrm{DF}_{\widehat{V}_{0}, K}\left(Y_{w}\right)$ too coincides with the Ross-Thomas orbifold Futaki invariant on $\left(M_{0}, L_{0}\right)$, see [18, Def. 5.2] and the discussion following it.

6.4. Sasaki K-stability versus weighted K-stability. Our main observation is the following result which links the relative Sasaki K-stability notion of [18] with the relative weighted K-stability of [40].

Proposition 6.9. Let $(N, \mathcal{D}, J, X)$ be a compact regular Sasaki manifold, and $(M, J, L)$ the corresponding smooth polarized Kähler manifold. Let $\mathbb{T} \leqslant \operatorname{Aut}(N, \mathcal{D}, J)$ be a compact torus with $X \in \mathfrak{t}_{+}$and $K \in \mathfrak{t}_{+}$another Sasaki-Reeb vector field on $(N, \mathcal{D}, J)$. Then, for any $\mathbb{T}$-equivariant smooth polarized test configuration $(\mathscr{M}, \mathscr{L})$ associated to $(M, J, L, \mathbb{T})$ we have

$$
\mathscr{F}_{K}(\mathscr{M}, \mathscr{L})=\frac{1}{2 \pi} \mathscr{G} \mathscr{F}_{K}(\mathscr{V}),
$$

where $\mathscr{F}_{K}(\mathscr{M}, \mathscr{L})$ is introduced by (25) .

Proof. The definition (21) can be rewritten as

$$
\begin{aligned}
\operatorname{Scal}_{\tilde{f}, m+2}(\tilde{\Omega}) \tilde{f}^{-m-3} & =\left(\operatorname{Scal}_{\tilde{f}, m+3}(\tilde{\Omega})+2 \tilde{f} \Delta_{\tilde{\Omega}} \tilde{f}+2(m+2)|d \tilde{f}|_{\tilde{\Omega}}^{2}\right) \tilde{f}^{-m-3} \\
& =\operatorname{Scal}_{\tilde{f}, m+3}(\tilde{\Omega}) \tilde{f}^{-m-3}+2 \delta_{\tilde{\Omega}}\left(\tilde{f}^{-m-2} d f\right) .
\end{aligned}
$$

\footnotetext{
${ }^{2}$ We are grateful to $\mathrm{X}$. Wang for pointing out to us this reference and to J. Ross for answering our questions.
} 
Hence by Stokes' Theorem

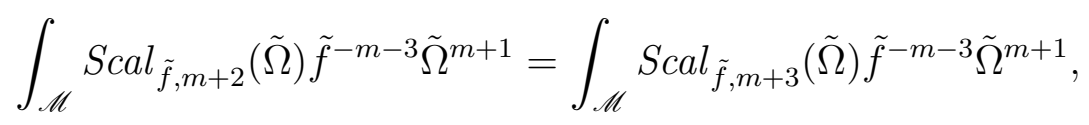

so that (25) is equivalently given by

$$
\mathscr{F}_{K}(\mathscr{M}, \mathscr{L})=-\int_{\mathscr{M}}\left(\operatorname{Scal}_{\tilde{f}}(\tilde{\Omega})-c_{K} \tilde{f}\right) \tilde{f}^{-3-m} \frac{\tilde{\Omega}^{m+1}}{(m+1) !}+2 \int_{\mathscr{M}} \tilde{f}^{-1-m} \pi^{*} \omega_{F S} \wedge \frac{\omega^{m}}{m !},
$$

where $\operatorname{Scal}_{\tilde{f}}(\tilde{\Omega})=\operatorname{Scal}_{\tilde{f}, m+1}(\tilde{\Omega})$ is $\tilde{f}$-scalar curvature of the Kähler manifold $(\mathscr{M}, \tilde{\Omega})$. Formula (46) tells us that on the Sasaki manifold $(\mathscr{N}, \mathscr{D}, \mathscr{J}, X)$ we have

$$
\tilde{\eta}_{\mathscr{D}}^{X}=\tilde{f} \tilde{\eta}_{\mathscr{D}}^{K}, \tilde{f}=\tilde{\eta}_{\mathscr{D}}^{X}(K), \operatorname{Scal}_{K}\left(\tilde{g}_{X}\right)=\operatorname{Scal}_{\tilde{f}}(\tilde{\Omega})=\tilde{f} \operatorname{Scal}\left(\tilde{g}_{K}\right)
$$

and we compute

$$
\begin{aligned}
\mathscr{F}_{K}(\mathscr{M}, \mathscr{L})= & -\int_{\mathscr{M}}\left(\operatorname{Scal}_{\tilde{f}}(\tilde{\Omega})-c_{K} \tilde{f}\right) \tilde{f}^{-3-m} \frac{\tilde{\Omega}^{m+1}}{(m+1) !}+2 \int_{\mathscr{M}} \tilde{f}^{-1-m} \pi^{*} \omega_{F S} \wedge \frac{\omega^{m}}{m !} \\
= & -\frac{1}{2 \pi(m+1) !} \int_{\mathscr{N}}\left(\operatorname{Scal}_{K}\left(\tilde{g}_{X}\right)-c_{K} \tilde{\eta}_{\mathscr{D}}^{X}(K)\right) \tilde{\eta}_{\mathscr{D}}^{X}(K)^{-3-m} \tilde{\eta}_{\mathscr{D}}^{X} \wedge\left(d \tilde{\eta}_{\mathscr{D}}^{X}\right)^{m+1} \\
& +\frac{2}{(2 \pi) m !} \int_{\mathscr{N}} \pi^{*}\left(\omega_{F S}\right) \wedge \tilde{\eta}_{\mathscr{D}}^{X}(K)^{-1-m} \tilde{\eta}_{\mathscr{D}}^{X} \wedge\left(d \tilde{\eta}_{\mathscr{D}}^{X}\right)^{m} \\
= & \frac{1}{2 \pi} \mathscr{G} \mathscr{F}(\mathscr{V}) .
\end{aligned}
$$

As a direct consequence of Propositions $6.8 \& 6.9$ and Theorem 5.5 we obtain the following ramification of the result in [18].

Corollary 6.10. Let $(V, K)$ be the polarized complex cone associated to a compact Sasaki manifold $(N, \mathcal{D}, J, K), \mathbb{T} \leqslant \operatorname{Aut}(V, J)^{K}$ a maximal torus with $K \in \mathfrak{t}_{+}$and assume that there exists a regular Sasaki-Reeb vector field $X \in \mathfrak{t}_{+}$. If $(V, K)$ admits a scalar-flat Kähler cone metric, then for any smooth $\mathbb{T}$-equivariant test configuration $(\mathscr{V}, K)$ of $(V, K)$, obtained from a smooth $\mathbb{T}$-equivariant polarized test configuration $(\mathscr{M}, \mathscr{L})$ associated to the regular quotient $(M, J, L, \mathbb{T})$, which has reduced central fibre and is not a product, we have $\mathrm{DF}_{\widehat{V}_{0}, K}\left(Y_{w}\right)>0$.

Proof. It is well-known (see e.g. [11) that a scalar-flat Kähler cone metric on $(V, J, K)$ corresponds to a CSC Sasaki structure in $\mathcal{S}\left(K, J^{K}\right)$, such that the constant $c_{K}$ defined by (48) equals $m(m+1)$. Thus, we are under the assumptions of Theorem 5.5 with $\ell_{\text {ext }}(x)=c_{K} \ell_{K}(x)$, i.e., $\mathscr{F}_{K}^{\text {ext }}(\mathscr{M}, \mathscr{L})=\mathscr{F}_{K}(\mathscr{M}, \mathscr{L})>0$. By Proposition 6.9 we have $\mathscr{G} \mathscr{F}_{K}(\mathscr{V})>0$ whereas Proposition 6.8 yields $\operatorname{DF}_{\widehat{V}_{0}, K}\left(Y_{w}\right)>0$.

\section{Applications}

In this section we solve the existence problem of extremal Sasaki metrics on a special class of CR manifolds of Sasaki type, extending Theorem 2 in [1] to arbitrary dimension.

7.1. The Calabi construction on admissible manifolds. This is the familiar construction of special Kähler metrics on $\mathbb{P}^{1}$ bundles, going back to Calabi [15] and studied in many other places.

We essentially follow the notation of [4] and consider $(M, J)=\mathbb{P}(\mathcal{O} \oplus L) \rightarrow B$ to be the total space of a $\mathbb{P}^{1}$-bundle over the product $B=B_{1} \times \cdots \times B_{k}$ of compact CSC Kähler manifolds $\left(B_{j}, J_{j}, g_{j}, \omega_{j}\right)$ satisfying the Hodge condition $\omega_{j}=2 \pi c_{1}\left(L_{j}\right)$ for a polarization $L_{j}$ on $B_{j}$, and $L=\bigotimes_{j=1}^{k} L_{j}^{p_{j}}$ with $p_{j} \in \mathbb{Z}$ being the induced line bundle on $B$. We fix 
real constants $c_{j} \in \mathbb{R}$ such that the affine linear functions $p_{j} z+c_{j}>0$ on the interval $P=[-1,1] \subseteq \mathbb{R}$ (equivalently, we require $c_{j}>\left|p_{j}\right|$ ).

Then, as shown for instance in [4], any smooth function $\Theta(z)$ on $[-1,1]$ satisfying the following boundary conditions

$$
\Theta( \pm 1)=0, \quad \Theta^{\prime}( \pm 1)=\mp 2,
$$

and

$$
\Theta(z)>0 \text { on }(-1,1) .
$$

gives rise to a Kähler metric on $M$ of the form

$$
\begin{aligned}
& g=\sum_{j=1}^{k}\left(p_{j} z+c_{j}\right) \pi^{*} g_{j}+\frac{d z^{2}}{\Theta(z)}+\Theta(z) \theta^{2}, \\
& \omega=\sum_{j=1}^{k}\left(p_{j} z+c_{j}\right) \pi^{*} \omega_{j}+d z \wedge \theta, d \theta=\sum_{j=1}^{k} p_{j} \pi^{*} \omega_{j},
\end{aligned}
$$

which is isometric to a Kähler metric in the same Kähler class $\zeta_{c}$ of $(M, J)$ (here $c=$ $\left(c_{1}, \ldots, c_{k}\right)$ stand for the fixed real constants in the definition (38) $)$. Notice that in (38), $\hat{\mathbf{\Theta}}$ is a connection 1 -form on the principal $\mathbb{S}^{1}$-bundle $P$ over $B$, corresponding to line bundle $L^{*}$, and $M=\mathbb{P}^{1} \times_{\mathbb{S}^{1}} P \rightarrow B$ is the corresponding fibre bundle associated to the toric variety $\left(\mathbb{P}^{1}, \mathcal{O}(2)\right)$. The induced $\mathbb{S}^{1}$-action on $M$ corresponds to fibrewise rotations in the trivial factor $\mathcal{O}$ of $\mathcal{O} \oplus L$, and (38) is $\mathbb{S}^{1}$-invariant with $m_{\omega}=z$ being the corresponding momentum map with image $P=[-1,1] \subseteq \mathbb{R}$. For any real constants $b>|a|, f(z)=a z+b$ is a positive Killing potential with respect to (38) for the Killing vector field $a \check{K}$ where $K$ is the generator of the $\mathbb{S}^{1}$-action, and the constant $b$ is essentially the normalization constant $\kappa>0$ of $\$ 3.2$. The $f$-scalar curvature of the Kähler metric (38) is computed in [6] to be

$$
\operatorname{Scal}_{f}(g)=-\frac{\left(f(z)^{-m-1} p_{c}(z) \Theta(z)\right)^{\prime \prime}}{p_{c}(z) f(z)^{-m-3}}+f^{2}(z) \sum_{j=1}^{k} \frac{S c a l_{j}}{p_{j} z+c_{j}}
$$

where $S c a l_{j}$ stands for the constant scalar curvature of $\left(B_{j}, g_{j}\right)$ and

$$
p_{c}(z):=\prod_{j=1}^{k}\left(p_{j} z+c_{j}\right)^{\operatorname{dim}_{\mathbb{C}} B_{j}}
$$

is a polynomial of degree $(m-1)$. By [6, Lemma 2.4], (38) gives rise to an $f$-extremal Kähler metric if and only if the smooth function $\Theta(z)$ on $[-1,1]$ satisfies (36), (37) and the condition

$$
\left(f(z)^{-m-1} p_{c}(z) \Theta(z)\right)^{\prime \prime}=p_{c}(z) f(z)^{-1-m}\left(\sum_{j=1}^{k} \frac{S c a l_{j}}{p_{j} z+c_{j}}\right)-(A z+B) f(z)^{-m-3} p_{c}(z)
$$

for some (unknown) real constants $A$ and $B$. The general solution of (40) thus depends on 4 real constants (including $A$ and $B$ ) and the 4 boundary conditions in (36) determine these constants uniquely, as it follows from the arguments of [6, Prop. 2.2]. We denote by $\ell_{a, b, c}^{\text {ext }}(z)=A_{a, b, c} z+B_{a, b, c}$ the corresponding affine-linear function and by $\Theta_{a, b, c}^{\text {ext }}(z)$ the corresponding solution of (40), verifying (36) but possibly not (37).

Up to a suitable renormalization, this is the setting of [4, 6], except that we allowed $p_{j}=0$ (which introduces a reducible manifold $(M, J)$ on which nontrivial solutions of the $f$-extremal equation can exist when $f \neq$ const), and did not introduce here blow-downs. Indeed, when $p_{j} \neq 0$, in order to get to the notation of [4], one simply needs to replace $\omega_{j}$ 
by (the possibly negative definite) CSC Kähler metric $p_{j} \omega_{j}$ and thus letting in (38) $p_{j}=1$ and $c_{j}=\frac{1}{x_{j}}, 0<\left|x_{j}\right|<1$.

Definition 7.1. [4] A Kähler manifold $(M, J)$ obtained as above is called admissible. The Kähler metric on it and the corresponding Kähler class $\zeta_{c}$ defined by (38) for some choice of $c=\left(c_{1}, \ldots, c_{k}\right)$ with $c_{j}>\left|p_{j}\right|$ and smooth function $\Theta(z)$ are called admissible.

Our main result relevant to the admissible setting is the following explicit Yau-TianDonaldson type correspondence.

Theorem 7.2. For an admissible Kähler manifold $\left(M, J, \zeta_{c}\right)$ as above, there exists an $\mathbb{S}^{1}$ invariant $(a \check{K}, b)$-extremal Kähler metric in the Kähler class $\zeta_{c}$ if and only if the function $\Theta_{a, b, c}^{\mathrm{ext}}(z)$ is positive on $(-1,1)$. In this case, $(M, J)$ admits an admissible $(a \check{K}, b)$-extremal Kähler metric of the form (38).

Theorem 7.2 is established in various special cases, but to the best of our knowledge was open in the above generality prior to this work. Indeed, in the non-weighted case (which correspond to $(a, b)=(0,1))$ Theorem 7.2 was first proved in 53 in the case of polarized ruled surfaces, and in [4] in general, whereas on a ruled complex surface and arbitrary $(a, b)$ with $|a|<b$ it is established in [1, Prop. 7]. Many other special cases are discussed in [6].

It is not hard to see that when the real constants $c_{1}, \ldots, c_{k}$ in (38) are all rational, the corresponding Kähler class $\zeta_{c}$ on $(M, J)$ belongs to $H^{2}(M, \mathbb{Q})$. Thus, for a suitable homothety factor $\lambda_{c}>0$, a Kähler metric $\left(\lambda_{c} g, \lambda_{c} \omega\right)$ given by (38) defines a smooth CR manifold $(N, \mathcal{D}, J)$ of Sasaki type (see Example 1.3) , endowed with a 2-dimensional torus $\mathbb{T} \leqslant \operatorname{Aut}(N, \mathcal{D}, J)$ and a regular Sasaki-Reeb element $X \in \mathfrak{t}_{+}$generating an $\mathbb{S}_{X}^{1} \leqslant \mathbb{T}$, such that $M$ is the Sasaki-Reeb quotient with respect to $X$ and has the induced $\check{\mathbb{T}}=\mathbb{T} / \mathbb{S}_{X}^{1}=\mathbb{S}_{\breve{K}}^{1}$ action generated by $\check{K}$. In other words, the choice of a positive Killing potential $f=$ $a z+b, b>\mid a]$ with respect to the Kähler metric $\left(\lambda_{c} g, \lambda_{c} \omega\right)$ defines an element $K_{a, b} \in \mathfrak{t}_{+}$, inducing $a \check{K}$ on $M$. In this setting, Proposition 3.7 and Theorem 7.2 yield the following generalization of [1, Theorem 2].

Corollary 7.3. $(N, \mathcal{D}, J)$ admits an extremal Sasaki metric in $\mathcal{S}\left(K_{a, b}, J^{K_{a, b}}\right)$ if and only if the corresponding smooth function $\Theta_{a, b, c}^{\mathrm{ext}}(z)>0$ on $(-1,1)$.

Notice that in Theorem 7.2 , the usually more difficult part, namely establishing the existence of an $(a \check{K}, b)$-extremal Kähler metric under a suitable positivity algebraic condition, is trivial because if $\Theta_{a, b, c}^{\text {ext }}(z)$ verifies (37), then letting $\Theta(z)=\Theta_{a, b, c}^{\text {ext }}(z)$ in (38) defines an admissible $(a K, b)$-extremal Kähler in $\zeta_{c}$. In the other direction, Lahdili shows in 40, Theorem 8] that for each $z_{0} \in(-1,1)$, one can find an $\mathbb{S}^{1}$-invariant smooth test configuration $\mathscr{M}$ which, as a complex manifold, is the degeneration to the normal cone [50] of $(M, J)$ with respect to the infinity section $B_{\infty}:=\mathbb{P}(\mathcal{O} \oplus 0) \subseteq M$, together with a Kähler class $\mathscr{A}_{z_{0}}$, such that the corresponding relative $(\check{K}, \kappa)$-weighted Donaldson-Futaki invariant introduced in $\$ 5.3$ satisfy

$$
\mathscr{F}_{a \tilde{K}, b}^{\operatorname{ext}}\left(\mathscr{M}, \mathscr{A}_{z_{0}}\right)=C\left(z_{0}\right) \Theta_{a, b, c}^{\mathrm{ext}}\left(z_{0}\right)
$$

for a positive function $C(z)$ on $(-1,1)$. Thus, Theorem [5.3 yields (see [40, Theorem 8]) that $\Theta_{a, b, c}^{\text {ext }}(z) \geq 0$ on $(-1,1)$ and the main difficulty in the proof of Theorem 7.2 is to establish the strict inequality in Theorem 5.3 for the test configurations $\left(\mathscr{M}, \mathscr{A}_{z_{0}}\right)$ as above. In particular, Conjecture 5.8 would imply Theorem 7.2 for $c$ rational.

7.2. The case of arbitrary weights. Before giving the proof of Theorem 7.2 , we will need to recall the more general setting of $(v, w)$-extremal Kähler metrics introduced in [40]. We limit the discussion to the $\mathbb{P}^{1}$-fibre bundle case, referring the Reader to [40] for the most general setting. Following [40, Section 10], let $v(z)$ and $w(z)$ be two positive 
smooth functions on $[-1,1]$, and let us look, more generally, for Kähler metrics on $M=$ $\mathbb{P}(\mathcal{O} \oplus L) \rightarrow B$ of the form (38), satisfying the equation

$$
\left(v(z) p_{c}(z) \Theta(z)\right)^{\prime \prime}=p_{c}(z) v(z)\left(\sum_{j=1}^{k} \frac{S c a l_{j}}{p_{j} z+c_{j}}\right)-(A z+B) w(z) p_{c}(z),
$$

for some (unknown) real constants $A, B$. If we set $v(z)=(a z+b)^{-m-1}$ and $w(z)=$ $(a z+b)^{-3-m}$ in (41), we obtain (40). For general positive weight functions $(v, w)$ defined over $P=[-1,1]$, (41) describes a $(v, w)$-extremal Kähler metric in the Kähler class $\zeta_{c}$ in the sense of [40], which is given by the Calabi construction (38).

Definition 7.4. [40] Let $v(z), w(z)$ be given positive smooth functions on $[-1,1]$ and $\tilde{\omega} \in \zeta_{c}$ an $\mathbb{S}^{1}$-invariant Kähler metric with corresponding normalized momentum map $m_{\tilde{\omega}}: M \rightarrow[-1,1]$. We say that $\tilde{\omega}$ is $(v, w)$-extremal if it satisfies

$$
\operatorname{Scal}_{v}(\tilde{g}):=v\left(m_{\tilde{\omega}}\right) \operatorname{Scal}(\tilde{g})+2 \Delta_{\tilde{g}} v\left(m_{\tilde{\omega}}\right)+v^{\prime \prime}\left(m_{\tilde{\omega}}\right) \tilde{g}(K, K)=\left(A m_{\tilde{\omega}}+B\right) w\left(m_{\tilde{\omega}}\right) .
$$

One can argue again that for $v(z), w(z)$ and $c=\left(c_{1}, \ldots, c_{k}\right)$ fixed, (41) admits a unique smooth solution $\Theta_{v, w, c}^{\text {ext }}(z)$, satisfying (36) but possibly not (37). Notice that the constants $A, B$ can be determined by integrating (41) on $[-1,1]$ against the affine-linear functions 1 and $z$. Integration by parts and using (36) yields a linear system for $A, B$, independent of $\Theta$. This leads to the notion of the extremal affine-linear function $\ell_{v, w, c}^{\text {ext }}(z):=A_{v, w, c} z+B_{v, w, c}$ associated to $\left(v, w, \zeta_{c}\right)$.

By establishing a suitable notion of relative $(v, w)$-K-stability, the following result is obtained in [40, Theorem 8].

Theorem 7.5. [40] If $\Theta_{v, w, c}^{\text {ext }}\left(z_{0}\right)<0$ for some $z_{0} \in(-1,1)$ then $(M, J)$ admits no $(v, w)$ extremal Kähler metric in $\zeta_{c}$.

We shall next improve the above statement under a technical assumption allowing to deduce Theorem 7.2 .

Theorem 7.6. If $\Theta_{v, w, c}^{\mathrm{ext}}(z)$ vanishes at $z_{0} \in(-1,1)$ but $\ell_{v, w, c}^{\text {ext }}\left(z_{0}\right) \neq 0$, then there is no $(v, w)$-extremal metric in $\zeta_{c}$.

Proof. Suppose $\Theta_{v, w, c}^{\text {ext }}\left(z_{0}\right)=0$ for some $z_{0} \in(-1,1)$ and $\ell_{v, w, c}^{\text {ext }}\left(z_{0}\right) \neq 0$, where $\ell_{v, w, c}^{\text {ext }}(z)=$ $A z+B$ is the extremal affine-linear function. Assume for contradiction that $\zeta_{c}$ admits a $(v, w)$-extremal Kähler metric. By Theorem 7.5, $\Theta_{v, w, c}^{\text {ext }}(z) \geq 0$ on $(-1,1)$. The idea is to violate Theorem 7.5 by varying the weights $(v, w)$.

Consider the smooth family of functions

$$
\Theta_{t}(z):=\Theta_{v, w, c}^{\mathrm{ext}}(z)-t\left(1-z^{2}\right)^{2}(A z+B)^{3} .
$$

By its very construction, $\Theta_{t}(z)$ satisfies (36) for each $t, \Theta_{0}(z)=\Theta_{v, w, c}^{\text {ext }}(z)$ and either for all $t>0$ or for all $t<0$ we have $\Theta_{t}\left(z_{0}\right)<0$. Suppose that $\Theta_{t}\left(z_{0}\right)<0$ for $t>0$.

Notice that $\Theta_{t}(x)$ solves (41) with respect to the weight functions $v(x)$ and

$$
w_{t}(z)=w(z)+t \tilde{w}(z), \quad \tilde{w}(z):=\frac{\left(v(z) p(z)\left(1-z^{2}\right)^{2}(A z+B)^{3}\right)^{\prime \prime}}{(A z+B) p(z)} .
$$

As $\tilde{w}(z)$ is a smooth function on $[-1,1], w_{t}(z)$ is positive and smooth on $[-1,1]$ for $t$ small enough. It follows that $\Theta_{t}(z)=\Theta_{v, w_{t}, c}^{\text {ext }}(z)$ is the unique solution of (41) for the weights $\left(v, w_{t}\right)$, satisfying (36). Using Theorem 7.5 again, we conclude that for small positive values of $t$, there is no $\left(v, w_{t}\right)$-extremal Kähler metric in $\zeta_{c}$. This contradicts Theorem B2 in [40]. 


\subsection{Proof of Theorem $\mathbf{7 . 2}$.}

Proof. As we have already explained, we are in the special case of Theorem 7.6 with $v(z)=(a z+b)^{-1-m}$ and $w(z)=(a z+b)^{-3-m}(b>|a|)$. Accordingly, it is enough to assume that $(M, J)$ admits an $(a K, b)$-extremal Kähler metric in $\zeta_{c}$ but $\Theta_{a, b, c}^{\text {ext }}\left(z_{0}\right)=\ell_{a, b, c}^{\text {ext }}\left(z_{0}\right)=0$ for some $z_{0} \in(-1,1)$, and derive a contradiction. To this end, we shall use a modification of the arguments from [4].

We start with the preliminary observation that $\Theta_{a, b, c}^{\text {ext }}(z)$ and $\ell_{a, b, c}^{\text {ext }}(z)$ are rational functions of $z$ whose coefficients are rational functions of $(a, b, c)$. Indeed, letting $P_{a, b, c}(z):=$ $p_{c}(z) \Theta_{a, b, c}^{\text {ext }}(z)$ and multiplying both sides of (41) with $(a z+b)^{m+3}$, it follows that $P_{a, b, c}^{\text {ext }}(z)$ solves the ODE

$$
\begin{aligned}
& (a z+b)^{2} F^{\prime \prime}(z)-2 a(m+1)(a z+b) F^{\prime}(z)+a^{2}(m+1)(m+2) F(z) \\
& =p_{c}(z)(a z+b)^{2}\left(\sum_{j=1}^{k} \frac{S c a l_{j}}{p_{j} z+c_{j}}\right)-(A z+B) p_{c}(z) .
\end{aligned}
$$

where $A$ and $B$ are real constants (equal to $A_{a, b, c}$ and $B_{a, b, c}$ for the specific solution $P_{a, b, c}(z)$ ). When $a \neq 0$, using that the monomials $(a z+b)^{s}, s=0, \ldots, m$ are eigenfunctions for nonzero eigenvalues of the linear differential operator in (43), we see that the general solution of (43) is a polynomial of degree $\leq m+2$ of the form

$$
F(z)=\sum_{s=0}^{m+2} p_{s}(a z+b)^{s}
$$

whose coefficients $p_{0}, \ldots, p_{m}$ are determined from $a, b, c$ and the two real constants $A, B$, whereas the coefficients $p_{m+2}$ and $p_{m+1}$ are the 2 free constants of integration. Imposing the boundary conditions (see (36))

$$
F( \pm 1)=0, F^{\prime}( \pm 1)=\mp 2 p_{c}( \pm 1)
$$

leads to a linear system for $\left(p_{m+2}, p_{m+1}, A, B\right)$. Under the geometric constraints $|a|<b$ and $c_{j}>\left|p_{j}\right|$ (and using that the setting is invariant under a homothety of $(a, b)$ ) it follows from the arguments in [6, Prop. 2.2] that the system admits a unique solution. This conclusion holds as long as the corresponding $4 \times 4$ system is nondegenerate. In summary, for the values of $(a, b, c)$ such that $a<|b|, a \neq 0, c_{j}>\left|p_{j}\right|, P_{a, b, c}(z)$ is a polynomial of degree $\leq m+2$, whose coefficients are rational functions of $a, b, c$, and, similarly, $\ell_{a, b, c}^{\text {ext }}(z)=A_{a, b, c} z+B_{a, b, c}$ is an affine-linear function whose coefficients are rational functions of $(a, b, c)$. The definition of $P_{a, b, c}(z)$ and $\ell_{a, b, c}^{\text {ext }}(z)$ can be also extended for $a=0, b>0$ and $c_{j}>\left|p_{j}\right|$ (e.g. by the arguments in the proof of [6, Prop. 2.2]) and for values $(a, b, c)$ in a maximal connected component $\mathcal{P}$ of the geometric solutions (i.e., satisfying $a<|b|, c_{j}>\left|p_{j}\right|$ ).

We next observe that under the assumption made, for all $(\tilde{a}, \tilde{b}, \tilde{c})$ sufficiently close to the initial value $(a, b, c),(M, J)$ admits a $(\tilde{a} \check{K}, \tilde{b})$-extremal Kähler metric in $\zeta_{\tilde{c}}$, $\Theta_{\tilde{a}, \tilde{b}, \tilde{c}}^{\operatorname{ext}}(z) \geq 0$ on $(-1,1)$ and $\Theta_{\tilde{a}, \tilde{b}, \tilde{c}}^{\text {ext }}\left(\tilde{z}_{0}\right)=0=\ell_{\tilde{a}, \tilde{b}, \tilde{c}}^{\text {ext }}\left(\tilde{z}_{0}\right)$ for some $\tilde{z}_{0} \in(-1,1)$. (This implies that $-\frac{B_{\tilde{a}, \tilde{b}, \tilde{c}}}{A_{\tilde{a}, \tilde{b}, \tilde{c}}}$ is a double root, possibly at infinity, of the degree $(m+2)$ polynomial $P_{\tilde{a}, \tilde{b}, \tilde{c}}(z)$.) Indeed, otherwise, by the openness result [40, Theorem B2], we can find a sequence $\lim _{k \rightarrow \infty}\left(a_{k}, b_{k}, c_{k}\right)=(a, b, c)$ such that $(M, J)$ admits an $\left(a_{k} \breve{K}, b_{k}\right)$-extremal Kähler metric in $\zeta_{c_{k}}$ (and therefore $\Theta_{a_{k}, b_{k}, c_{k}}^{\text {ext }}(z) \geq 0$ on $(-1,1)$ by Theorem 7.5$)$ but $\Theta_{a_{k}, b_{k}, c_{k}}^{\text {ext }}(z)$ and $\ell_{a_{j}, b_{j}, c_{j}}^{\text {ext }}(z)$ do not having a common zero in $(-1,1)$. By Theorem 7.6 , $\Theta_{a_{k}, b_{k}, c_{k}}^{\text {ext }}(z)>0$ on $(-1,1)$, i.e., there exist an admissible $\left(a_{k} \check{K}, b_{k}\right)$-extremal Kähler metrics $\left(g_{k}, \omega\right)$ in $\zeta_{c_{k}}$ of the form (38). The latter are, by the results in [3], precisely the Kähler metrics on $(M, J)$ admitting a hamiltonian 2 -form of order 1 with associated Killing vector field $\check{K}$. Using 
the uniqueness result of [41] (see also Lemma 4.9 in this paper), we conclude as in the proof of Theorem 2 in [4] that the initial $(a \check{K}, b)$-extremal metric in $\zeta_{c}$ must also admit a hamiltonian 2 -form of order 1 associated to $\check{K}$, a contradiction.

The conclusion, which will are going to use in order to find a contradiction, is that (by analytic continuation) for all $(\tilde{a}, \tilde{b}, \tilde{c}) \in \mathcal{P}, P_{\tilde{a}, \tilde{b}, \tilde{c}}(z)$ is a polynomial of degree $\leq(m+2)$, which has a double root at $-\frac{B_{\tilde{a}, \tilde{b}, \tilde{c}}}{A_{\tilde{a}, \tilde{b}, \tilde{c}}}$, i.e.,

$$
\left(A_{\tilde{a}, \tilde{b}, \tilde{c}}\right)^{m+2} P_{\tilde{a}, \tilde{b}, \tilde{c}}\left(-\frac{B_{\tilde{a}, \tilde{b}, \tilde{c}}}{A_{\tilde{a}, \tilde{b}, \tilde{c}}}\right)=0, \quad\left(A_{\tilde{a}, \tilde{b}, \tilde{c}}\right)^{m+1} P_{\tilde{a}, \tilde{b}, \tilde{c}}^{\prime}\left(-\frac{B_{\tilde{a}, \tilde{b}, \tilde{c}}}{A_{\tilde{a}, \tilde{b}, \tilde{c}}}\right)=0 .
$$

We thus reduced the proof to test (45) in the extended domain of definition $\mathcal{P}$ of $(a, b, c)$ and show that (45) cannot hold for all such values of the parameters. We are going to specialize (45) for $a=1=b$. In this case, the system (44) for the general solution of (44) is still nondegenerate. Indeed, the boundary conditions at $z=-1$ of the general solution hold if and only if $A_{1,1, c}=B_{1,1, c}=-2 m(m+1)$ which in turn identifies all but the coefficients $p_{m+2}$ and $p_{m+1}$ of the general solutions of (43). The boundary conditions at $z=1$ thus fix the constants $p_{m+2}$ and $p_{m+1}$. In particular, $(1,1, c) \in \mathcal{P}$. Notice that by (44), $z=-1$ is a single zero of $P_{1,1, c}(z)$, which shows that the second equality in (45) does not hold.

\section{Appendix A. Holomorphic Versus contact VIEWPOINT}

For any compact co-oriented contact $(2 m+1)$-manifold $(N, \mathcal{D})$, let $\mathcal{C}_{+}(N, \mathcal{D})^{\mathbb{T}}$ be the space of $\mathbb{T}$-invariant $\mathrm{CR}$ structures $J$ on $\mathcal{D}$, and let

$$
\mathfrak{c o n}_{+}(N, \mathcal{D})=\left\{X \in \mathfrak{c o n}(N, \mathcal{D}) \mid \eta_{\mathcal{D}}(X)>0\right.
$$

(using the chosen orientation of $T N / \mathcal{D}$ ). Given a (compact, real) torus $\mathbb{T}$ in $\operatorname{Con}(N, \mathcal{D})$ let $\operatorname{Con}(N, \mathcal{D})^{\mathbb{T}}$ denote the group of $\mathbb{T}$-equivariant contact transformations, with Lie algebra $\mathfrak{c o n}(N, \mathcal{D})^{\mathbb{T}}$ (in which the Lie algebra $\mathfrak{t}$ of $\mathbb{T}$ is central).

Let $\left(N, \mathcal{D}_{0}, J_{0}, X\right)$ be a fixed Sasaki manifold invariant under a torus $\mathbb{T}$, let $\eta_{0}:=\eta_{\mathcal{D}_{0}}^{X}$ be its contact form, and denote the induced transversal holomorphic structure on $\mathcal{D}_{X}$ by $J_{0}^{X}$. We consider the following two ways of parametrizing a family of CR structures on $N$.

- The contact viewpoint refers to the space $\mathcal{C}_{0}\left(X, \mathcal{D}_{0}\right)^{\mathbb{T}} \subseteq \mathcal{C}_{+}\left(N, \mathcal{D}_{0}\right)^{\mathbb{T}}$ of $\mathbb{T}$-invariant $\mathrm{CR}$ structures $J$ on $\mathcal{D}_{0}$ such that the induced transversal holomorphic structure $J^{X}$ is the pushforward of $J_{0}^{X}$ by some $\Phi \in \operatorname{Diff}_{0}(N)^{\mathbb{T}}$. Note that $J$ is uniquely determined by $J^{X}$ (via the given fixed $\mathcal{D}_{0}$ and $X$ ), and it is sometimes convenient to view $T_{J} \mathcal{C}_{+}\left(N, \mathcal{D}_{0}\right)^{\mathbb{T}}$ as a space of endomorphisms of $\mathcal{D}_{X}$ rather than $\mathcal{D}_{0}$.

- The (transversal) holomorphic viewpoint refers to the space $\mathcal{S}\left(X, J_{0}^{X}\right)^{\mathbb{T}}$ of $\mathbb{T}$-invariant contact forms $\eta=\eta_{\mathcal{D}}^{X}$ which are compatible with the transversal holomorphic structure $\left(X, J_{0}^{X}\right)$, and hence induce a $\mathrm{CR}$ structure $(\mathcal{D}, J)$ where $J$ is the lift of $J_{0}^{X}$ to $\mathcal{D}$.

Note that the groups $\operatorname{Con}_{0}\left(N, \mathcal{D}_{0}\right)^{\mathbb{T}}:=\operatorname{Con}\left(N, \mathcal{D}_{0}\right) \cap \operatorname{Diff}_{0}(N)^{\mathbb{T}}$ and $\operatorname{Aut}_{0}\left(N, X, J_{0}^{X}\right)^{\mathbb{T}}:=$ $\operatorname{Aut}\left(N, X, J_{0}^{X}\right) \cap \operatorname{Diff}_{0}(N)^{\mathbb{T}}$ act on $\mathcal{C}_{0}\left(X, \mathcal{D}_{0}\right)^{\mathbb{T}}$ and $\mathcal{S}\left(X, J_{0}^{X}\right)^{\mathbb{T}}$ respectively by pushforward. Imitating arguments in 31, we have the following Sasaki analogue of a fundamental result in Kähler geometry.

Proposition A.1. The relation $\sim$ between $\mathcal{C}_{0}\left(X, \mathcal{D}_{0}\right)^{\mathbb{T}}$ and $\mathcal{S}\left(X, J_{0}^{X}\right)^{\mathbb{T}}$, defined by $J \sim \eta$ if and only if there exists $\Phi \in \operatorname{Diff}_{0}(N)^{\mathbb{T}}$ such that $\left(\mathcal{D}_{0}, J^{X}\right)=\Phi \cdot\left(\mathcal{D}, J_{0}^{X}\right)$, where $\mathcal{D}=\operatorname{ker} \eta$, induces respectively $\operatorname{Con}_{0}\left(N, \mathcal{D}_{0}\right)^{\mathbb{T}}$ and $\operatorname{Aut}_{0}\left(N, X, J_{0}^{X}\right)^{\mathbb{T}}$ invariant maps

$$
\begin{aligned}
& p: \mathcal{C}_{0}\left(X, \mathcal{D}_{0}\right)^{\mathbb{T}} \rightarrow \mathcal{S}\left(X, J_{0}^{X}\right)^{\mathbb{T}} / \operatorname{Aut}_{0}\left(N, X, J_{0}^{X}\right)^{\mathbb{T}} \text { and } \\
& q: \mathcal{S}\left(X, J_{0}^{X}\right)^{\mathbb{T}} \rightarrow \mathcal{C}_{0}\left(X, \mathcal{D}_{0}\right)^{\mathbb{T}} / \operatorname{Con}_{0}\left(N, \mathcal{D}_{0}\right)^{\mathbb{T}},
\end{aligned}
$$


which define a bijection

$$
\mathcal{C}_{0}\left(X, \mathcal{D}_{0}\right)^{\mathbb{T}} / \operatorname{Con}_{0}\left(N, \mathcal{D}_{0}\right)^{\mathbb{T}} \cong \mathcal{S}\left(X, J_{0}^{X}\right)^{\mathbb{T}} / \operatorname{Aut}_{0}\left(N, X, J_{0}^{X}\right)^{\mathbb{T}} .
$$

The derivative of $p$ at $J$, with $J^{X}=\Phi \cdot J_{0}^{X}$, is given by $d p_{J}\left(-\mathcal{L}_{Y} J^{X}\right)=\Phi^{*}\left(\mathcal{L}_{Y} \eta_{0}\right)$ modulo $\mathfrak{a u t}\left(N, X, J_{0}^{X}\right)^{\mathbb{T}}$, and the derivative of $q$ at $\eta=\Psi \cdot \eta_{0}^{X}$ is given by $d q_{\eta}\left(-\mathcal{L}_{Y} \eta\right)=\Psi^{*}\left(\mathcal{L}_{Y} J_{0}^{X}\right)$ modulo $\operatorname{con}\left(N, \mathcal{D}_{0}\right)^{\mathbb{T}}$.

Proof. Suppose $J \sim \eta$; then $\tilde{J} \sim \eta$ if and only if there exists $\Phi \in \operatorname{Diff}_{0}(N)^{\mathbb{T}}$ with

$$
\Phi \cdot\left(\mathcal{D}_{0}, J^{X}\right)=\left(\mathcal{D}_{0}, \tilde{J}^{X}\right) \text {, i.e., } \Phi \in \operatorname{Con}_{0}\left(N, \mathcal{D}_{0}\right)^{\mathbb{T}} \text { and } \Phi \cdot J=\tilde{J} .
$$

Similarly, $J \sim \tilde{\eta}$, with ker $\tilde{\eta}=\tilde{\mathcal{D}}$ if and only if there exists $\Psi \in \operatorname{Diff}_{0}(N)^{\mathbb{T}}$ with

$$
\Psi \cdot\left(\mathcal{D}, J_{0}^{X}\right)=\left(\tilde{\mathcal{D}}, J_{0}^{X}\right) \text {, i.e., } \Psi \in \operatorname{Aut}_{0}\left(N, X, J_{0}^{X}\right)^{\mathbb{T}} \text { and } \Psi \cdot \eta=\tilde{\eta}
$$

(since $\Psi$ preserves $X$ ). Hence the relation $J \sim \eta$ induces invariant and uniquely defined, but perhaps only partially defined, $p$ and $q$. However, $p$ is everywhere defined by definition of $\mathcal{C}_{0}\left(X, \mathcal{D}_{0}\right)^{\mathbb{T}}$, while $q$ is everywhere defined by the equivariant Gray-Moser theorem (cf. Remark 1.8). Thus $p$ and $q$ are invariant maps as stated, and descend to mutually inverse maps on respective quotients by construction.

To compute $d p_{J}\left(-\mathcal{L}_{Y} J^{X}\right)$, let $\Phi_{t}$ be a curve in $\operatorname{Diff}_{0}(N)^{\mathbb{T}}$ with $\Phi_{0}=\Phi$ and $\left.\frac{d}{d t} \Phi_{t} \Phi^{-1}\right|_{t=0}=$ $Y$ and let $J_{t}$ be the curve in $\mathcal{C}_{0}\left(X, \mathcal{D}_{0}\right)^{\mathbb{T}}$ with $J_{t}^{X}=\Phi_{t} \cdot J_{0}^{X}$; thus $J_{t}$ passes through $J$ at $t=0$ with derivative (the lift to $\mathcal{D}_{0}$ of) $-\mathcal{L}_{Y} J^{X}$. Then $p\left(J_{t}\right)=\Phi_{t}^{-1} \cdot \eta_{0}=\Phi^{*}\left(\Phi_{t} \Phi^{-1}\right)^{*} \eta_{0}$ modulo $\operatorname{Aut}_{0}\left(N, X, J_{0}^{X}\right)^{\mathbb{T}}$ and so

$$
d p_{J}\left(-\mathcal{L}_{Y} J^{X}\right)=\Phi^{*}\left(\left.\frac{d}{d t}\left(\Phi_{t} \Phi^{-1}\right)^{*} \eta_{0}\right|_{t=0}\right)=\Phi^{*}\left(\mathcal{L}_{Y} \eta_{0}\right)
$$

modulo $\mathfrak{a} \mathfrak{u}\left(N, X, J_{0}^{X}\right)^{\mathbb{T}}$. The derivative of $q$ is computed similarly, or by using the inverse relationship between $p$ and $q$.

Remark A.2. We may describe the derivatives of $p$ and $q$ more explicitly using Lemma 1.7,

(i) If $\mathcal{L}_{Y} J^{X}$ is tangent to $\mathcal{C}_{0}\left(X, \mathcal{D}_{0}\right)^{\mathbb{T}}$ at $J^{X}$, its lift to $\mathcal{D}_{0}$ is skew with respect to $d \eta_{0}$. However, this lift differs from $\mathcal{L}_{Y} J$ by a 1 -form with values in the span of $X$ (on which $d \eta_{0}$ vanishes), so $\mathcal{L}_{Y} J$ is skew with respect to $d \eta_{0}$. Since $J$ is also skew with respect to $d \eta_{0}$ it follows that $J$ is skew with respect to $d\left(\mathcal{L}_{Y} \eta_{0}\right)=\mathcal{L}_{Y} d \eta_{0}$; hence $d\left(\mathcal{L}_{Y} \eta_{0}\right)$ is $J^{X}$-invariant, so $\mathcal{L}_{Y} \eta_{0}$ is tangent to $\mathcal{S}\left(X, J^{X}\right)^{\mathbb{T}}$ at $\eta_{0}$ hence of the form $d_{X}^{J} \varphi+\alpha$ by Lemma 1.7 .

Hence, modulo aut $\left(N, X, J_{0}^{X}\right)^{\mathbb{T}}, d p_{J}\left(-\mathcal{L}_{Y} J\right) \equiv \Phi^{*}\left(\mathcal{L}_{Y} \eta_{0}\right)=\mathcal{L}_{\Phi^{*} Y} \eta=d_{X}^{J_{0}} \psi+\beta$, with $\psi=\Phi^{*} \varphi$ and $\beta=\Phi^{*} \alpha$. Since $\mathfrak{o}(N, X)^{\mathbb{T}} \leqslant \mathfrak{a u t}\left(N, X, J_{0}^{X}\right)^{\mathbb{T}}$ induces exact forms $d f$ for $\mathbb{T}$-invariant functions $f$, we only need to determine $\beta$, and hence $\alpha$, modulo such exact forms. Thus we may take $d^{J} \varphi+\alpha=\iota_{Y} d \eta_{0}$, since this differs from $\mathcal{L}_{Y} \eta_{0}$ by such an exact form. Thus $d p_{J}\left(-\mathcal{L}_{Y} J\right)=d_{X}^{J_{0}} \psi+\beta=\Phi^{*} \iota_{Y} d \eta_{0}$ modulo aut $\left(N, X, J_{0}^{X}\right)^{\mathbb{T}}$.

(ii) Conversely the derivative of $q$ at $\eta=\Psi \cdot \eta_{0}=\Phi^{*} \eta_{0}$, with $\Psi=\Phi^{-1}$, sends $d_{X}^{J_{0}}\left(\Phi^{*} \varphi\right)+\Phi^{*} \alpha$ to $-\mathcal{L}_{Y} J^{X}$ modulo $\mathfrak{c o n}_{0}\left(N, \mathcal{D}_{0}\right)^{\mathbb{T}}$, where $\iota_{Y} d \eta_{0}=d_{X}^{J} \varphi+\alpha$. We check that this has tangent vectors of the form $d f$ in its kernel, since they correspond to elements of $\mathfrak{c o n}_{0}\left(N, \mathcal{D}_{0}\right)^{\mathbb{T}}$. On the other hand, applied to a tangent vector of the form $d_{X}^{J_{0}}\left(\Phi^{*} \varphi\right)$, we obtain instead $-\mathcal{L}_{Y} J^{X}$ where $\iota_{Y} d \eta_{0}=d_{X}^{J} \varphi$ modulo $\mathfrak{c o n}_{0}\left(N, \mathcal{D}_{0}\right)^{\mathbb{T}}$. Thus $Y=-J X_{\varphi}$ modulo the span of $X$, where $X_{\varphi}$ is the contact vector field of $\varphi$ with respect to $\eta_{0}$, and we conclude that $d q_{\eta}\left(d_{X}^{J_{0}}\left(\Phi^{*} \varphi\right)\right)=-\mathcal{L}_{Y} J^{X}=\mathcal{L}_{J^{X}{ }_{X}} J^{X}=J^{X} \circ \mathcal{L}_{X_{\varphi}} J^{X}$ modulo $\mathfrak{c o n}_{0}\left(N, \mathcal{D}_{0}\right)^{\mathbb{T}}$.

Thus the image of the marking $\Xi\left(X, J^{X}, \eta_{0}\right)$ under $q$ may be interpreted as (the quotient by $\operatorname{Con}_{0}\left(N, \mathcal{D}_{0}\right)^{\mathbb{T}}$ of $)$ an orbit for a complexification of $\operatorname{Con}_{0}\left(N, \mathcal{D}_{0}\right)^{\mathbb{T}}$, even though such a complexification does not exist. 


\section{Appendix B. The CONTACT Futaki invariant}

Let $(N, \mathcal{D})$ be a compact co-oriented contact $(2 m+1)$-manifold and $\mathbb{T}$ a fixed compact real torus in its group $\operatorname{Con}(N, \mathcal{D})$ of contact transformations. The group $\operatorname{Con}(N, \mathcal{D})^{\mathbb{T}}$ (with Lie algebra $\left.\operatorname{con}(N, \mathcal{D})^{\mathbb{T}}\right)$ of $\mathbb{T}$-equivariant contact transformations naturally acts on the space $\mathcal{C}_{+}(N, \mathcal{D})^{\mathbb{T}}$ of $\mathbb{T}$-invariant $\mathrm{CR}$ structures on $\mathcal{D}$, compatible with the fixed orientation on $T N / \mathcal{D}$ (which we assume is nonempty). Using Lemma 1.1, we identify $\mathfrak{c o n}(N, \mathcal{D})^{\mathbb{T}}$ with the space $C_{N}^{\infty}(T N / \mathcal{D})^{\mathbb{T}}$ of smooth $\mathbb{T}$-invariant sections of $T N / \mathcal{D}$. Fix $K \in \mathfrak{t} \cap \mathfrak{c o n}_{+}(N, \mathcal{D})$ where $\mathfrak{t}$ is the Lie algebra of $\mathbb{T}$. It is shown in [1] that for any CR structure $J \in \mathcal{C}_{+}(N, \mathcal{D})^{\mathbb{T}}$ and any $X \in \mathfrak{t} \cap \mathfrak{c o n}_{+}(N, \mathcal{D})$,

$$
\operatorname{Scal}_{K}\left(g_{X}\right) \tau=\operatorname{Scal}\left(g_{K}\right) \sigma=: \operatorname{Scal}_{\sigma}(J),
$$

where $\sigma:=\eta_{\mathcal{D}}(K), \tau:=\eta_{\mathcal{D}}(X)$. It follows that $\operatorname{Scal}_{\sigma}(J)$ is a well-defined element of $C_{N}^{\infty}(T N / \mathcal{D})^{\mathbb{T}}$; furthermore, following [1], the linear map

$$
\begin{aligned}
\mu_{\sigma}(J): C_{N}^{\infty}(T N / \mathcal{D})^{\mathbb{T}} & \rightarrow \mathbb{R} \\
\rho & \mapsto\left\langle\left\langle S c a l_{\sigma}(J), \rho\right\rangle_{\sigma}\right.
\end{aligned}
$$

where for $\sigma_{1}, \sigma_{2} \in C_{N}^{\infty}(T N / \mathcal{D})^{\mathbb{T}}$

$$
\left\langle\left\langle\sigma_{1}, \sigma_{2}\right\rangle\right\rangle_{\sigma}:=\int_{N} \sigma_{1} \sigma_{2} \sigma^{-m-3} \operatorname{vol}_{\mathcal{D}}
$$

with $\operatorname{vol}_{\mathcal{D}}:=\eta_{\mathcal{D}} \wedge L_{\mathcal{D}}^{\wedge m}$ denoting the section of $\wedge^{2 m+1} T^{*} N \otimes(T N / \mathcal{D})^{m+1}$, can be identified with the momentum map for the action of $\operatorname{Con}(N, \mathcal{D})^{\mathbb{T}}$ on the space $\mathcal{C}_{+}(N, \mathcal{D})^{\mathbb{T}}$, with respect to a formal symplectic structure constructed in [32].

This provides a conceptual explanation for Lemma 3.2. Notice that for a $J \in \mathcal{C}_{+}(N, \mathcal{D})^{\mathbb{T}}$, $(\mathcal{D}, J, K)$ is extremal Sasaki if and only if the contact vector field $X_{S c a l}(J) \in \mathfrak{c r}(N, \mathcal{D}, J)$ whereas $(\mathcal{D}, J, K)$ is CSC Sasaki if and only if

$$
X_{\operatorname{Scal}_{\sigma}(J)}=c_{K} K
$$

where $c_{K}$ is the constant

$$
c_{K}:=\frac{\left\langle\left\langle S c a l_{\sigma}(J), \sigma\right\rangle\right\rangle_{\sigma}}{\langle\langle\sigma, \sigma\rangle\rangle_{\sigma}}=\frac{\int_{N} \operatorname{Scal}\left(g_{K}\right) \eta_{\mathcal{D}}^{K} \wedge\left(d \eta_{\mathcal{D}}^{K}\right)^{m}}{\int_{N} \eta_{\mathcal{D}}^{K} \wedge\left(d \eta_{\mathcal{D}}^{K}\right)^{m}} .
$$

We now use the above setting to give a definition of a contact Futaki invariant Fut F $_{K}: \mathfrak{t} \rightarrow$ $\mathbb{R}$ associated to $(N, \mathcal{D}, \mathbb{T})$, which obstructs the existence of a CSC Sasaki structure in $\mathcal{C}_{+}(N, \mathcal{D})^{\mathbb{T}}$, thus providing a contact analogue of the construction in [43]. Compared to the constructions in [12, 29], using Remark 1.8, Fut ${ }_{K}$ coincides with the restriction to the Lie algebra $\mathfrak{t} \leqslant \mathfrak{a u t}\left(N, K, J^{K}\right)$ of the invariant defined in these references with respect to the transversal holomorphic structure $\left(N, K, J^{K}\right)$.

The point is that the flow of a vector field $Y \in \mathfrak{t} \leqslant \mathfrak{c o n}(N, \mathcal{D})^{\mathbb{T}}$ acts trivially on the space $\mathcal{C}_{+}(N, \mathcal{D})^{\mathbb{T}}$ and, therefore, by the momentum map interpretation of (47), we have that the quantity

$$
\left\langle\left\langle\operatorname{Scal}_{\sigma}(J), \rho\right\rangle\right\rangle_{\sigma}
$$

where $\rho=\eta_{\mathcal{D}}(Y) \in C_{N}^{\infty}(T N / \mathcal{D})$ is independent of $J \in \mathcal{C}_{+}(N, \mathcal{D})^{\mathbb{T}}$. In particular, the constant $c_{K}$ defined by (48) and the $\langle\langle\cdot, \cdot\rangle\rangle_{\sigma}$-orthogonal projection $\sigma_{\text {ext }}$ of $\operatorname{Scal}_{\sigma}(J)$ to the space $C_{N}^{\infty}(T N / \mathcal{D})$ are independent of $J$. We denote by $K_{\text {ext }} \in \mathfrak{t}$ the vector field corresponding to $\sigma_{\text {ext }}$ via Lemma 1.1, and for any $K_{1}, K_{2} \in \mathfrak{c o n}(N, \mathcal{D})^{\mathbb{T}}$ with corresponding sections $\sigma_{1}, \sigma_{2} \in C_{N}^{\infty}(T N / \mathcal{D})^{\mathbb{T}}$, we let

$$
\left\langle\left\langle K_{1}, K_{2}\right\rangle\right\rangle_{K}:=\left\langle\left\langle\sigma_{1}, \sigma_{2}\right\rangle\right\rangle_{\sigma}=\int_{N} \eta_{\mathcal{D}}^{K}\left(K_{1}\right) \eta_{\mathcal{D}}^{K}\left(K_{2}\right) \eta_{\mathcal{D}}^{K} \wedge\left(d \eta_{\mathcal{D}}^{K}\right)^{m} .
$$

The above prompts the following definition. 
Definition B.1. Given a compact Sasaki manifold $(N, \mathcal{D}, J, K)$, a compact torus $\mathbb{T} \subseteq$ $\operatorname{Aut}(N, \mathcal{D}, J)$ with Lie algebra $\mathfrak{t} \subseteq \mathfrak{c o n}(N, \mathcal{D})$ such that $K \in \mathfrak{t}$, we define a linear map Fut $_{K}: \mathfrak{t} \rightarrow \mathbb{R}$ by

$$
\operatorname{Fut}_{K}(Z):=\left\langle\left\langle X_{S c a l_{\sigma}(J)}-c_{K} K, Z\right\rangle\right\rangle_{K},
$$

where the constant $c_{K}$ is given by (48). We also write

$$
\operatorname{Fut}_{K}(Z)=\left\langle\left\langle K_{\mathrm{ext}}-c_{K} K, Z\right\rangle\right\rangle_{K}
$$

where $K_{\text {ext }} \in \mathfrak{t}$ is the $\langle\langle\cdot, \cdot\rangle\rangle_{K^{-}}$orthogonal projection of $X_{\text {Scal }}(J)$ to $\mathfrak{t}$, called extremal vector field of $(K, \mathbb{T})$ (cf. [35] in the case when $\mathbb{T}$ is maximal and [42] in the toric case).

As Fut $_{K}$ is independent of $J \in \mathcal{C}_{+}(N, \mathcal{D})^{\mathbb{T}}$, it follows that the condition Fut $_{K} \equiv 0$ (or equivalently $\left.K_{\text {ext }}=c_{K} K\right)$ is necessary for finding a CR structure $J \in \mathcal{C}_{+}(N, \mathcal{D})^{\mathbb{T}}$ which is CSC with respect to $K$.

Remark B.2. In the case when $X$ is quasiregular, the Futaki invariant Fut $K$ can be rewritten in terms of the corresponding compact Kähler orbifold $(M, J, \omega)$ as

$$
\operatorname{Fut}_{K}(Z)=(2 \pi) \int_{M}\left(\operatorname{Scal}_{f_{K}}(g)-c_{K} f_{K}\right) f_{Z} f_{K}^{-m-3} \omega^{m}
$$

where $f_{Z}=\eta_{\mathcal{D}}^{X}(Z)$ and $f_{K}=\eta_{\mathcal{D}}^{X}(K)$ are the induced Killing potentials on $M$. This is, up to a positive dimensional constant, the Futaki invariant of [40], associated to the Kähler class $\zeta=[\omega]$ on $(M, J)$, the induced torus action $\check{\mathbb{T}}$ with momentum polytope $P_{X}$, and the weight functions $v(x)=\ell_{K}(x)^{-m-1}$ and $w(x)=\ell_{K}(x)^{-m-2}$ (where $\ell_{K}(x)$ is the positive affine-linear function over $P_{X}$ corresponding to $K$, see Lemma 1.15).

\section{ACKNOWLEDGEMENTS}

VA was supported in part by an NSERC Discovery Grant and is grateful to the University of Bath and the Institute of Mathematics and Informatics of the Bulgarian Academy of Sciences for their support and hospitality. EL was supported by the French ANR-14-CE250010 and is grateful to the UQAM and the UMI-CNRS in Montreal for their support and hospitality. We thank R. Dervan, A. Lahdili, J. Ross, Z. Sjöström Dyrefelt and X. Wang for valuable discussions.

\section{REFERENCES}

[1] V. Apostolov and D. M. J. Calderbank, The CR geometry of weighted extremal Kähler and Sasaki metrics, arXiv:1810.10618, to appear in Math. Ann., https://doi.org/10.1007/s00208-020-02095-1.

[2] V. Apostolov, D. M. J. Calderbank, P. Gauduchon and E. Legendre, Levi-Kähler reduction of CR structures, product of spheres, and toric geometry, arXiv:1708.05253, to appear in Math. Res. Lett.

[3] V. Apostolov, D. M. J. Calderbank, P. Gauduchon and C. W. Tønnesen-Friedman, Hamiltonian 2-forms in Kähler geometry II Global Classification, J. Differential Geom. 68 (2004), 277-345.

[4] V. Apostolov, D. M. J. Calderbank, P. Gauduchon and C. W. Tønnesen-Friedman, Hamiltonian 2-forms in Kähler geometry III Extremal metrics and stability, Invent. Math. 173 (2008), 547-601.

[5] V. Apostolov and G. Maschler, Conformally Kähler, Einstein-Maxwell geometry, J. Eur. Math. Soc. 21 (2019), 1319-1360.

[6] V. Apostolov, G. Maschler and C. W. Tønnesen-Friedman, Weighted extremal Kähler metrics and the Einstein-Maxwell geometry of projective bundles, arXiv:1808.02813, to appear in Comm. Anal. Geom.

[7] R. Berman and B. Berndtsson, Convexity of the K-energy on the space of Kähler metrics and uniqueness of extremal metrics. J. Amer. Math. Soc. 30 (2017), 1165-1196.

[8] R. Berman, T. Darvas, and C. Lu, Regularity of weak minimizers of the K-energy and applications to properness and K-stability, Ann. Sci. Ec. Norm. Super. 53 (2020), 267-289.

[9] B. Berndtsson, A Brunn-Minkowski type inequality for Fano manifolds and some uniqueness theorems in Kähler geometry, Invent. Math. 200 (2015) 149-200.

[10] S. Boucksom, T. Hisamoto and M. Jonsson, Uniform K-stability, Duistermaat-Heckman measures and singularities of pairs, Ann. Inst. Fourier 67 (2017), 743-841. 
[11] C. P. Boyer and K. Galicki, Sasakian Geometry, Oxford Mathematical Monographs. Oxford University Press, Oxford, 2008.

[12] C. P. Boyer, K. Galicki and S. Simanca, Canonical Sasakian metrics, Comm. Math. Phys. 279 (2008), 705-733.

[13] C. P. Boyer, H. Huang, E. Legendre, An application of the Duistertmaat-Heckman theorem and its extensions in Sasaki geometry, Geometry \& Topology, 22 (2018), 4205-4234.

[14] C. P. Boyer and C. van Coevering, Relative K-stability and extremal Sasaki metrics, Math. Res. Lett. 28 (2018), 1-19.

[15] E. Calabi, Extremal Kähler metrics, Seminar on Differential Geometry, pp. 259-290, Ann. of Math. Stud. 102, Princeton Univ. Press, Princeton, N.J., 1982.

[16] X. X. Chen, M. Paun and Yu Zeng, On deformation of extremal metrics, arXiv:1506.01290.

[17] K. Cho, A. Futaki and H. Ono, Uniqueness and examples of compact toric Sasaki-Einstein metrics, Comm. Math. Phys. 277 (2008), 439-458.

[18] T. Collins and G. Székelyhidi, K-semistability for irregular Sasaki manifolds, J. Differential Geom. 109 (2018), 81-109.

[19] T. Collins and G. Székelyhidi, Sasaki-Einstein metrics and K-stability, Geom. Topol. 23 (2019), 13391413.

[20] T. Darvas, The Mabuchi geometry of finite energy class, Adv. Math. 285 (2015), 182-219.

[21] T. Darvas and Y. Rubinstein, Tian's properness conjectures and Finsler geometry of the space of Kähler metrics, J. Amer. Math. Soc. 3 (2017), 347-387.

[22] L. David, Weyl connections and curvature properties of CR manifolds, Ann. Global Anal. Geom. 26 (2004), 59-72.

[23] R. Dervan and J. Ross, K-stability for Kähler manifolds, Math. Res. Lett. 24 (2017), 689-739.

[24] S. K. Donaldson, Remarks on gauge theory, complex geometry and 4-manifold topology, Fields Medallists lectures, 384-403, World Sci. Ser. 20th Century Math., 5, World Sci. Publishing, River Edge, NJ, 1997.

[25] S. K. Donaldson, Scalar curvature and stability of toric varieties, J. Diff. Geom. 62 (2002), $289-349$.

[26] A. El Kacimi-Alaoui Opérateurs transversalement elliptiques sur un feuilletage riemannien et applications, Compositio Math. 73 (1990), 57-106.

[27] S. Falcão B. de Moraes and C. Tomei, Moment maps on symplectic cones, Pacific J. Math. 181 (1997), $357-375$.

[28] A. Futaki and H. Ono, Conformally Einstein-Maxwell Kähler metrics and structure of the automorphism group, Math. Z. 292 (2019), 571-589.

[29] A. Futaki, H. Ono and G. Wang, Transverse Kähler geometry of Sasaki manifolds and toric SasakiEinstein manifolds, J. Differential Geom. 83 (2009), 585-635.

[30] D. H. Phong and J. Sturm, Test configurations for K-stability and geodesic rays, J. Symp. Geom. 5 (2007), 221-247.

[31] P. Gauduchon, Calabi's extremal metrics: An elementary introduction, Lecture Notes.

[32] W. He, On the transverse scalar curvature of a compact Sasaki manifold, Complex Manifolds 1 (2014), $52-63$.

[33] W. He, On Calabi's extremal metric and properness, Trans. Amer. Math. Soc. 372 (2019), 5595-5619.

[34] W. He, Scalar curvature and properness on Sasaki manifolds, arXiv:1802.03841.

[35] W. He and J. Li, Geometric pluripotential theory on Sasaki manifolds, arXiv:1803.0068, to appear in J. Geom. Anal. https://doi.org/10.1007/s12220-019-00257-5.

[36] W. He and S. Sun, Frankel conjecture and Sasaki geometry, Adv. Math. 291 (2016), 912-960.

[37] F.C. Kirwan, Cohomology of Quotients in Complex and Algebraic Geometry, Mathematical Notes 31, Princeton University Press, Princeton N. J., 1984.

[38] A. Lahdili, Automorphisms and deformations of conformally Kähler, Einstein-Maxwell metrics, J. Geom. Anal. 29 (2019), 542-568.

[39] A. Lahdili, Conformally Kähler, Einstein-Maxwell metrics and boundedness of the modified Mabuchi functional, arXiv:1710.00235, to appear in Int. Math. Res. Notices.

[40] A. Lahdili, Kähler metrics with weighted constant scalar curvature and weighted K-stability, Proc. London Math. Soc. 119 (2019), 1065-114.

[41] A. Lahdili, Convexity of the weighted Mabuchi functional and the uniqueness of weighted extremal metrics, arXiv: 2007.01345.

[42] E. Legendre, Existence and non-uniqueness of constant scalar curvature toric Sasaki metrics, Compos. Math. 147 (2011), 1613-1634.

[43] M. Lejmi, Extremal almost-Kähler metrics, Internat. J. Math. 21 (2010), 1639-1662. 
[44] E. Lerman, A convexity theorem for torus actions on a contact manifold, Illinois J. Math. 46 (2002), 171-184.

[45] C. Li and C. Xu, Stability of Valuations: Higher Rational Rank, Peking Math J. 1 (2018), 1-79.

[46] D. Martelli, J. Sparks and S.-T. Yau, The geometric dual of a-maximisation for toric Sasaki-Einstein manifolds, Comm. Math. Phys. 268 (2006), 39-65.

[47] D. Martelli, J. Sparks and S.-T. Yau, Sasaki-Einstein manifolds and volume minimisation, Comm. Math. Phys. 280 (2008), 611-673.

[48] Y. Odaka, A genealization of the Ross-Thomas slope theory, Osaka J. Math. 50 (2013), 171-185.

[49] M. Reid, Graded rings and varieties in weighted projective space, lecture notes, 2002, URL:http://homepages.warwick.ac.uk/ masda/surf/more/grad.pdf.

[50] J. Ross and R. Thomas, Weighted projective embeddings, stability of orbifolds, and constant scalar curvature Kähler metrics, J. Differential Geom. 88 (2011), 109-159.

[51] Z. Sjöström Dyrefelt, K-semistability of cscK manifolds with transendental cohomology class, J. Geom. Anal. 28 (2018), 2927-2960.

[52] Z. Sjöström Dyrefelt (with an Appendix by R. Dervan), On K-polystability of CSCK manifolds with transcendental cohomology class, Int. Math. Res. Notices 2020 (2020), 2769-2817.

[53] G. Székelyhidi, Extremal metrics and K-stability, Bull. London Math. Soc. 39 (2007), 76-84.

[54] G. Tian, The K-energy on hypersurfaces and stability, Comm. Anal. Geom. 2 (1994), 239-265.

[55] G. Tian, Kähler-Einstein metrics with positive scalar curvature, Inventiones Math. 130 (1997), 1-37.

[56] C. van Coevering, Stability of Sasaki-extremal metrics under complex deformations, Int. Math. Res. Notices 24 (2013), 5527-5570.

[57] C. van Coevering, Monge-Ampère operators, energy functionals, and uniqueness of Sasaki-extremal metrics, arXiv:1511.09167.

[58] C. van Coevering, Examples of asymptotically conical Ricci-flat Kähler manifolds, Math. Z. 267 (2011), 465-496.

[59] X. Wang, Height and GIT weight, Math. Res. Lett. 18 (2012), 909-926.

[60] S. M. Webster, On the pseudo-conformal geometry of a Kähler manifold, Math. Z. 157 (1977), 265270 .

[61] S. T. Yau, Open problems in Geometry. In: "Differential Geometry: Partial Differential Equations on Manifolds". Proc. Sympos. Pure Math., vol. 54, pp. 1-28. AMS, Los Angeles, CA (1990).

[62] B. Zhou and X. Zhu, Relative K-stability and modified K-energy on toric manifolds, Adv. Math. 219 (2008), 1327-1362.

Vestislav Apostolov, Département de Mathématiques, UQAM, C.P. 8888, Succursale Centre-ville, Montréal (QuéBec), H3C 3P8, Canada

Email address: apostolov.vestislav@uqam.ca

David M. J. Calderbank, Department of Mathematical Sciences, University of Bath, BATH BA2 7AY, UK

Email address: D.M.J.Calderbank@bath.ac.uk

Eveline Legendre, Institut de Mathématiques de Toulouse, Université Paul Sabatier, 118 Route De NARbonne, 31062 Toulouse, France

Email address: eveline.legendre@math.univ-toulouse.fr 